Supporting Information for:

\title{
Nickel-Catalyzed Formal Aminocarbonylation of Unactivated Alkyl Iodides with Isocyanides
}

Wenyi Huang, Yun Wang, Yangyang Weng, Mohini Shrestha, Jingping Qu*, and Yifeng Chen*

Key Laboratory for Advanced Materials and Joint International Research Laboratory of Precision Chemistry and Molecular Engineering, Feringa Nobel Prize Scientist Joint Research Center, School of Chemistry and Molecular Engineering, East China University of Science \& Technology, 130 Meilong Road, Shanghai, 200237, China.

E-mail: qujp@dlut.edu.cn; yifengchen@ecust.edu.cn 


\section{Table of Contents}

General Information.

S-3

Optimization of the Ni-catalyzed Aminocarbonylation of Alkyl Iodides.........................S-4

Synthesis and Characterization of New Substrates....................................................

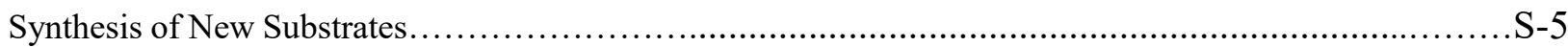

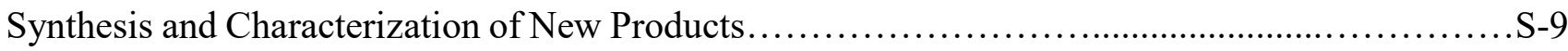

General Procedure for the Aminocarbonylation of Alkyl Iodides.......................................................

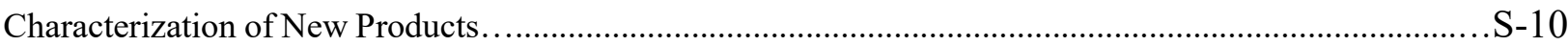

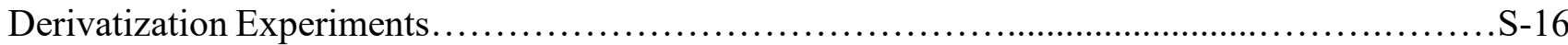

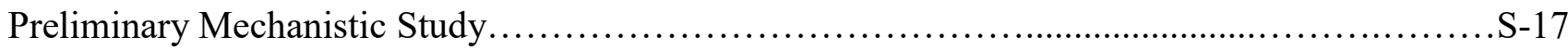

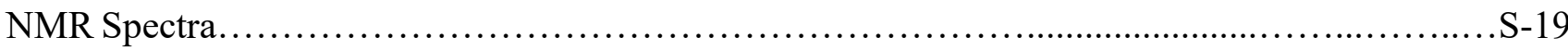

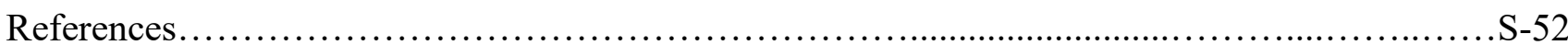




\section{General information}

All reactions were carried out under nitrogen atmosphere and anhydrous conditions unless otherwise indicated. All manipulations of air-sensitive or moisture-sensitive compounds were performed in a glovebox under an atmosphere of nitrogen. Unless otherwise noted, all catalytic reactions were run in dried glassware. Toluene and THF were distilled from sodium/benzophenone. DCM was distilled over $\mathrm{CaH}_{2}$. Ni(COD) $)_{2}$ (CAS 1295-35-8) was purchased from Sinocompound; IMes $\bullet \mathrm{HCl}$ was purchased from Energy Chemical; ${ }^{t} \mathrm{BuNC}(7188-38-7)$ was purchased from Meryer. Reactions were monitored by thin-layer chromatography (TLC) carried out on $0.20 \mathrm{~mm}$ Huanghai silica gel plates (HSGF 254) using UV light as the visualizing agent and an acidic solution of Phosphomolybdic Acid (PMA) and an acid solution of $p$-Anisaldehyde (PA) with heat as the stains. All new compounds were characterized by means of GC, ${ }^{1} \mathrm{H}-\mathrm{NMR},{ }^{13} \mathrm{C}-\mathrm{NMR}$, and HR-MS. GC analysis was performed on Agilent Technologies 7820A GC system. GC runs were performed with the following method: GC; HP-5 column; inlet temperature $250{ }^{\circ} \mathrm{C}$; column temperature $100{ }^{\circ} \mathrm{C}$ for $1 \mathrm{~min}$, then $50{ }^{\circ} \mathrm{C} / \mathrm{min}$ to $280{ }^{\circ} \mathrm{C}$, then $280{ }^{\circ} \mathrm{C}$ for $6 \mathrm{~min}$. NMR spectra were recorded using a Bruker AVANCE III $400 \mathrm{MHz}$ NMR spectrometer and can be found at the end of the paper. High-resolution mass spectra (HRMS) were recorded on a Waters GCT Premier mass spectrometer using EI-TOF (electron ionization-time of flight) or on a JEOC AccuTOF LC-plus 4G mass spectrometer using ESI (electrospray ionization). All ${ }^{1} \mathrm{H}-\mathrm{NMR}$ data are reported in $\delta$ units, parts per million (ppm), and were calibrated relative to the signals for residual chloroform $(7.26 \mathrm{ppm})$ in deuterochloroform $\left(\mathrm{CDCl}_{3}\right)$. All ${ }^{13} \mathrm{C}$-NMR data are reported in $\mathrm{ppm}$ relative to $\mathrm{CDCl}_{3}(77.16 \mathrm{ppm})$ and were obtained with ${ }^{1} \mathrm{H}$ decoupling. The following abbreviations or combinations thereof were used to explain the multiplicities: $\mathrm{s}=$ singlet, $\mathrm{d}=$ doublet, $\mathrm{t}=$ triplet, $\mathrm{q}=$ quartet, quin $=$ quintet, $\mathrm{sext}=$ sextet, $\mathrm{br}=$ broad, $\mathrm{m}=$ multiplet. 


\section{Optimization of the Ni-catalyzed Aminocarbonylation of Alkyl lodides}

Table S1. Optimization of the Ni-catalyzed Aminocarbonylation of Primary Alkyl Iodides 1a with ${ }^{t} \mathrm{BuNC}^{a}$

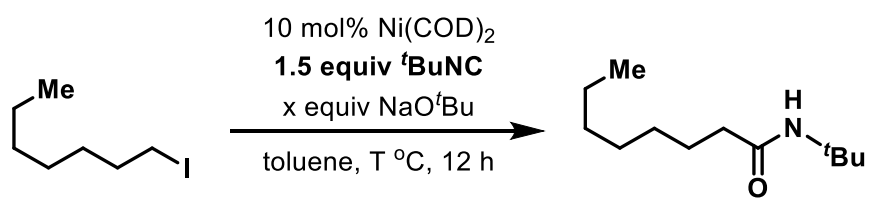

$1 \mathbf{a}$

$3 \mathbf{a}$

\begin{tabular}{lllll}
\hline Entry & $\mathbf{x}$ & Solvent & $\mathbf{T}^{\circ} \mathbf{C}$ & $\mathbf{3 a}[\%]^{b}$ \\
\hline 1 & 3.0 & toluene & 50 & 80 \\
2 & 2.0 & toluene & 50 & $83(99)^{c}$ \\
3 & 1.5 & toluene & 50 & 78 \\
4 & 3.0 & toluene & r.t. & 60 \\
5 & 3.0 & toluene & 100 & 80 \\
$6^{d}$ & 2.0 & toluene & 50 & 73 \\
7 & 2.0 & ${ }^{6} \mathrm{BuOH} / \mathrm{H}_{2} \mathrm{O}=10 / 1$ & 50 & 73 \\
\hline
\end{tabular}

${ }^{a}$ Reaction conditions: 1a $(0.1 \mathrm{mmol})$, tert-butyl isocyanide $(0.15 \mathrm{mmol}), \mathrm{Ni}(\mathrm{COD})_{2}(0.01 \mathrm{mmol}), \mathrm{NaO}{ }^{t} \mathrm{Bu}(0.2 \mathrm{mmol})$, toluene $(1.0$ $\mathrm{mL})$, at $50{ }^{\circ} \mathrm{C}, 12 \mathrm{~h}$. Then $1 \mathrm{M} \mathrm{HCl}(1.0 \mathrm{~mL})$, r.t., $5 \mathrm{~min} .{ }^{b}$ Corrected GC yield with dodecane as an internal standard. ${ }^{c}$ Isolated yield. ${ }^{d} 1.2$ equiv ${ }^{t} \mathrm{BuNC}$.

Table S2. Optimization of the Ni-catalyzed Aminocarbonylation of Secondary Alkyl Iodides 1q with ${ }^{t} \mathrm{BuNC}^{a}$

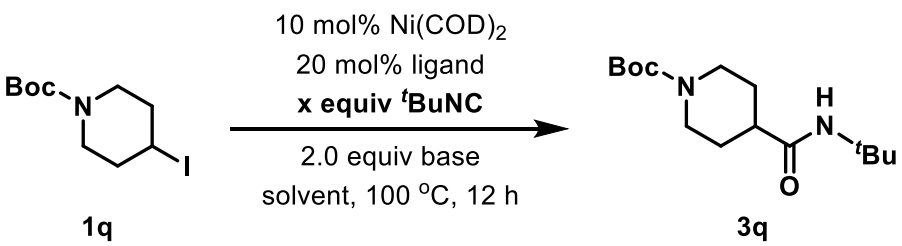

\begin{tabular}{llllll}
\hline Entry & Base & Ligand & $\mathbf{x}$ & Solvent & 3q [\%] ${ }^{\boldsymbol{b}}$ \\
\hline 1 & $\mathrm{NaO}{ }^{t} \mathrm{Bu}$ & $\mathrm{IPr} \cdot \mathrm{HCl}$ & 1.0 & toluene & 16 \\
2 & $\mathrm{KO}^{t} \mathrm{Bu}$ & $\mathrm{IPr} \cdot \mathrm{HCl}$ & 1.0 & toluene & - \\
3 & $\mathrm{LiO}^{t} \mathrm{Bu}$ & $\mathrm{IPr} \cdot \mathrm{HCl}$ & 1.0 & toluene & - \\
4 & $\mathrm{NaOMe}$ & $\mathrm{IPr} \cdot \mathrm{HCl}$ & 1.0 & toluene & 13 \\
5 & $\mathrm{Na}_{2} \mathrm{CO}_{3}$ & $\mathrm{IPr} \cdot \mathrm{HCl}$ & 1.0 & toluene & 7 \\
6 & $\mathrm{NaOEt}$ & $\mathrm{IPr} \cdot \mathrm{HCl}$ & 1.0 & toluene & 4 \\
7 & $\mathrm{NaO}{ }^{t} \mathrm{Bu}$ & $\mathrm{SIPr} \cdot \mathrm{HCl}$ & 1.0 & toluene & 26 \\
8 & $\mathrm{NaO}{ }^{t} \mathrm{Bu}$ & $\mathrm{IMes} \cdot \mathrm{HCl}$ & 1.0 & toluene & 25 \\
9 & $\mathrm{NaO}{ }^{t} \mathrm{Bu}$ & $\mathrm{SIMes} \cdot \mathrm{HCl}$ & 1.0 & toluene & 33 \\
10 & $\mathrm{NaO}{ }^{t} \mathrm{Bu}$ & $\mathrm{ICy} \cdot \mathrm{HCl}$ & 1.0 & toluene & - \\
11 & $\mathrm{NaO}{ }^{t} \mathrm{Bu}$ & $\mathrm{BPhen}$ & 1.0 & toluene & - \\
12 & $\mathrm{NaO}{ }^{t} \mathrm{Bu}$ & dppf & 1.0 & toluene & - \\
13 & $\mathrm{NaO}{ }^{t} \mathrm{Bu}$ & dppe & 1.0 & toluene & - \\
14 & $\mathrm{NaO}{ }^{t} \mathrm{Bu}$ & $\mathrm{PPh}$ & 1.0 & toluene & 51 \\
15 & $\mathrm{NaO}{ }^{t} \mathrm{Bu}$ & IMes $\cdot \mathrm{HCl}$ & 1.2 & toluene & \\
\hline
\end{tabular}




\begin{tabular}{|c|c|c|c|c|c|}
\hline Entry & Base & Ligand & $\mathbf{x}$ & Solvent & $3 q[\%]^{b}$ \\
\hline 16 & $\mathrm{NaO}^{t} \mathrm{Bu}$ & IMes'HCl & 1.5 & toluene & 26 \\
\hline 17 & $\mathrm{NaO}^{t} \mathrm{Bu}$ & dtbpy & 1.2 & toluene & 8 \\
\hline 18 & $\mathrm{NaO}{ }^{t} \mathrm{Bu}$ & IMes' $\mathrm{HCl}$ & 1.2 & o-xylene & 24 \\
\hline 19 & $\mathrm{NaO}^{t} \mathrm{Bu}$ & IMes'HCl & 1.2 & benzene & 24 \\
\hline 20 & $\mathrm{NaO}^{t} \mathrm{Bu}$ & IMes'HCl & 1.2 & dioxane & - \\
\hline 21 & $\mathrm{NaO}{ }^{t} \mathrm{Bu}$ & IMes' $\mathrm{HCl}$ & 1.2 & ${ }^{t} \mathrm{BuOH}$ & 59 \\
\hline 22 & $\mathrm{NaO}{ }^{t} \mathrm{Bu}$ & IMes'HCl & 1.2 & ${ }^{t} \mathrm{BuOH} /$ toluene $=1 / 1$ & 34 \\
\hline 23 & $\mathrm{NaO}^{t} \mathrm{Bu}$ & IMes'HCl & 1.2 & ${ }^{t} \mathrm{BuOH} /$ toluene $=2 / 1$ & 60 \\
\hline 24 & $\mathrm{NaO}^{t} \mathrm{Bu}$ & IMes' $\mathrm{HCl}$ & 1.2 & ${ }^{t} \mathrm{BuOH}\left(\right.$ add 2.0 equiv $\mathrm{H}_{2} \mathrm{O}$ ) & 69 \\
\hline 25 & $\mathrm{NaO}{ }^{t} \mathrm{Bu}$ & IMes' $\mathrm{HCl}$ & 1.2 & toluenen $/ \mathrm{H}_{2} \mathrm{O}=10 / 1$ & 47 \\
\hline 26 & $\mathrm{NaO}^{t} \mathrm{Bu}$ & IMes' $\mathrm{HCl}$ & 1.2 & ${ }^{t} \mathrm{BuOH} /$ toluene $/ \mathrm{H}_{2} \mathrm{O}=5 / 5 / 1$ & 83 \\
\hline 27 & $\mathrm{NaO}{ }^{t} \mathrm{Bu}$ & IMes $\cdot \mathrm{HCl}$ & 1.2 & ${ }^{t} \mathrm{BuOH} / \mathrm{H}_{2} \mathrm{O}=10 / 1$ & $92(92)^{c}$ \\
\hline
\end{tabular}

${ }^{a}$ Reaction conditions: 1q $(0.1 \mathrm{mmol})$, tert-butyl isocyanide $(0.12 \mathrm{mmol}), \mathrm{Ni}(\mathrm{COD}) 2(0.01 \mathrm{mmol}), \mathrm{IMes} \bullet \mathrm{HCl}(0.02 \mathrm{mmol}), \mathrm{NaO}{ }^{t} \mathrm{Bu}$ $(0.2 \mathrm{mmol}),{ }^{t} \mathrm{BuOH}(1.0 \mathrm{~mL}), \mathrm{H}_{2} \mathrm{O}(0.1 \mathrm{~mL})$ at $100{ }^{\circ} \mathrm{C}, 12 \mathrm{~h}$. Then $1 \mathrm{M} \mathrm{HCl}(1.0 \mathrm{~mL})$, r.t., $5 \mathrm{~min} .{ }^{b} \mathrm{Corrected} \mathrm{GC}$ yield with dodecane as an internal standard. ${ }^{c}$ Isolated yield.

Table S3. Optimization of the Ni-catalyzed Aminocarbonylation of Primary Alkyl Iodides 1a with PhNC ${ }^{a}$

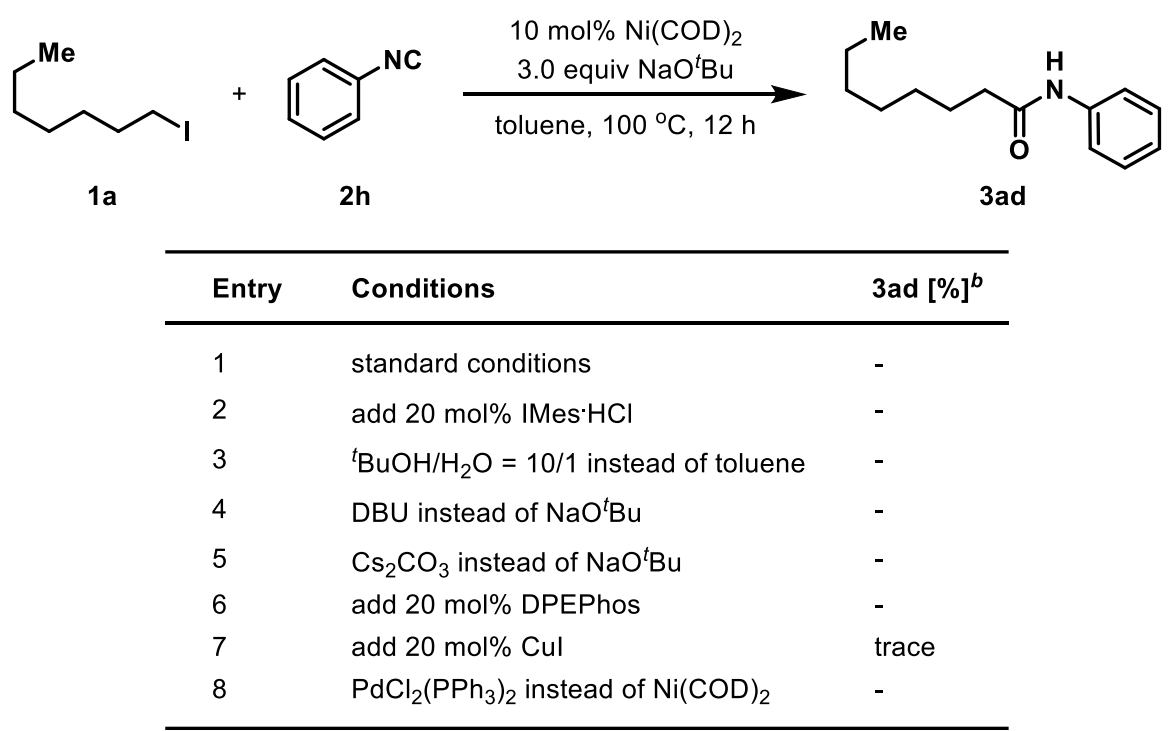

${ }^{a}$ Reaction conditions: 1a $(0.1 \mathrm{mmol})$, phenyl isocyanide $(0.15 \mathrm{mmol}), \mathrm{Ni}(\mathrm{COD})_{2}(0.01 \mathrm{mmol}), \mathrm{NaO}^{t} \mathrm{Bu}(0.3 \mathrm{mmol})$, toluene $(1.0 \mathrm{~mL})$, at $100{ }^{\circ} \mathrm{C}, 12 \mathrm{~h}$. Then $1 \mathrm{M} \mathrm{HCl}(1.0 \mathrm{~mL})$, r.t., $5 \mathrm{~min} .{ }^{b}$ Corrected GC yield with dodecane as an internal standard.

\section{Synthesis and Characterization of New Substrates}

\section{Synthesis of New Substrates}

Compounds $\mathbf{1 b},{ }^{[1]} \mathbf{1 c},{ }^{[2]} \mathbf{1 d},{ }^{[3]} \mathbf{1 e},{ }^{[4]} \mathbf{1 f},{ }^{[5]} \mathbf{1 g},{ }^{[6]} \mathbf{1 h},{ }^{[7]} \mathbf{1 i},{ }^{[8]} \mathbf{1 j},{ }^{[9]} \mathbf{1 q},{ }^{[10]} \mathbf{1 r},{ }^{[10]} \mathbf{1 s},{ }^{[5]} \mathbf{1 u},{ }^{[11]} \mathbf{1 v},{ }^{[10]}$ $\mathbf{2 b},{ }^{[12]} \mathbf{2} \mathbf{c},{ }^{[13]} \mathbf{2 d},{ }^{[14]} \mathbf{2 e},{ }^{[15]} \mathbf{2 f},{ }^{[16]} \mathbf{2 g},{ }^{[17]} \mathbf{2 h}^{[18]}$ were synthesized according to the published procedures. Substrate scope of isocyanides 


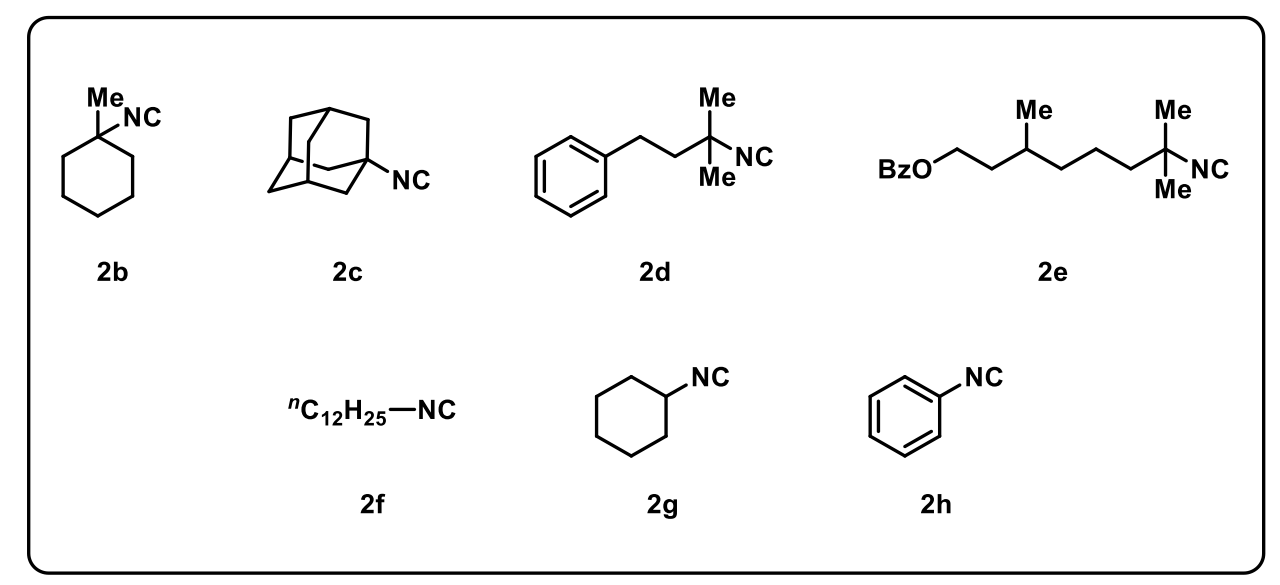

\section{General procedure A for substituted alkyl iodides synthesis.}

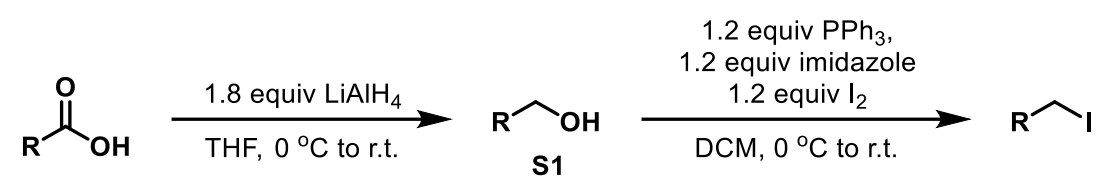

To a solution of the required acid (1.0 equiv) dissolved in THF $(0.33 \mathrm{M})$ was added $\mathrm{LiAlH}_{4}(1.8$ equiv) in small portions at $0^{\circ} \mathrm{C}$. The reaction mixture was then stirred at room temperature overnight. The reaction was quenched with $1 \mathrm{M}$ aq. $\mathrm{NaOH}$. After stirring for $15 \mathrm{~min}$, the reaction mixture was diluted with ethyl acetate, filtered through a celite plug and the solids washed thoroughly with ethyl acetate. The combined organic layer was washed with brine, dried over $\mathrm{Na}_{2} \mathrm{SO}_{4}$, and then filtered. The filtrate was concentrated in vacuo and the residue was purified by flash column chromatography on silica gel to afford the corresponding alkyl alcohol S1.

To a solution of $\mathrm{PPh}_{3}$ (1.2 equiv) and imidazole (1.2 equiv) in DCM (0.2 M) was added S1 (1.0 equiv) at $0{ }^{\circ} \mathrm{C}$, then $\mathrm{I}_{2}$ (1.2 equiv) was added in small portions. The mixture was slowly warmed to room temperature. The reaction mixture was stirred at room temperature until complete by TLC analysis. When the starting material was consumed, the reaction was quenched with the addition of sat. aq. $\mathrm{Na}_{2} \mathrm{~S}_{2} \mathrm{O}_{3}$ and extracted three times with DCM. The combined organic layer was dried over $\mathrm{Na}_{2} \mathrm{SO}_{4}$, and then filtered. The filtrate was concentrated in vacuo and the residue was purified by flash column chromatography on silica gel to afford the corresponding alkyl iodide.

Procedure for the synthesis of the alkyl iodide 1n

$(8 R, 9 S, 13 S, 14 S)-3-(4-i o d o b u t o x y)-13-m e t h y l-6,7,8,9,11,12,13,14,15,16-d e c a h y d r o-17 H-$ cyclopenta[a]phenanthren-17-one (1n)

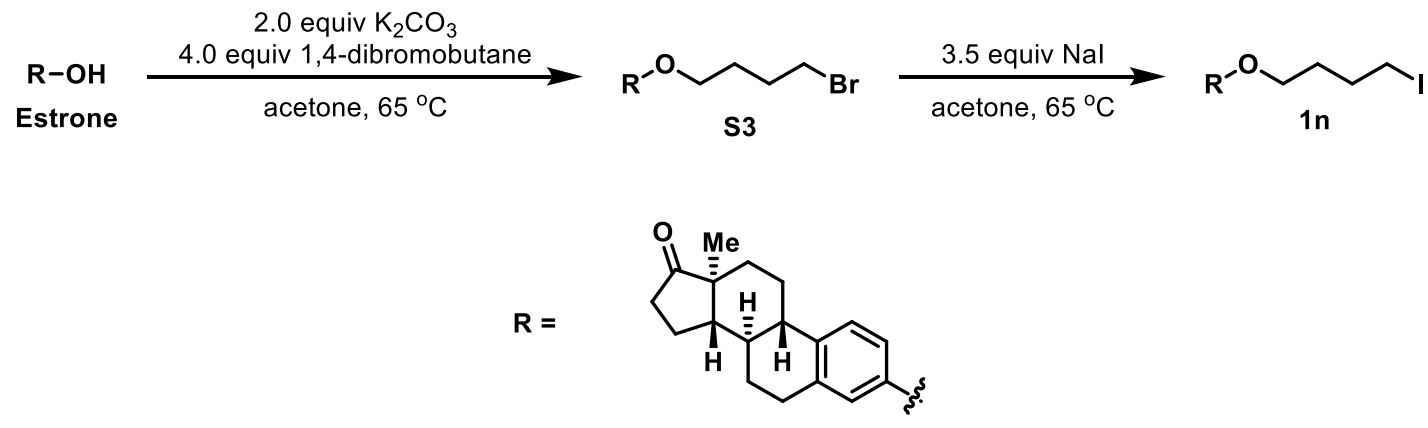


To a solution of Estrone (1.0 equiv, $10 \mathrm{mmol}, 2.7 \mathrm{~g}$ ) and potassium carbonate ( 2.0 equiv, $20 \mathrm{mmol}$, $2.8 \mathrm{~g}$ ) in anhydrous acetone $(0.25 \mathrm{M}, 40 \mathrm{~mL}$ ) was added 1,4-dibromobutane (4.0 equiv, $40 \mathrm{mmol}, 4.8$ $\mathrm{mL}$ ). The reaction mixture was stirred at a $65^{\circ} \mathrm{C}$ oil bath overnight. Then potassium carbonate was removed by filtration and the filtrate was concentrated in vacuo to afford $\mathbf{S 3}$.

To a solution of $\mathrm{NaI}$ (3.5 equiv, $35 \mathrm{mmol}, 5.2 \mathrm{~g})$ in acetone $(0.25 \mathrm{M}, 40 \mathrm{~mL})$ was added $\mathbf{S 3}(1.0$ equiv), and then the reaction mixture was stirred at a $65{ }^{\circ} \mathrm{C}$ oil bath overnight. After cooling at room temperature, the mixture was diluted with water and extracted three times with ethyl acetate. The combined organic layer was washed with sat. aq. $\mathrm{Na}_{2} \mathrm{~S}_{2} \mathrm{O}_{3}$ and brine, dried over $\mathrm{Na}_{2} \mathrm{SO}_{4}$ and then filtered. The filtrate was concentrated in vacuo and the residue was purified by flash column chromatography on silica gel to afford $\mathbf{1 n}$ as a white solid (over two steps, $2.7 \mathrm{~g}, 60 \%$ ), $\mathbf{R}_{\mathbf{f}}=0.52$ $(\mathrm{PE} / \mathrm{EtOAc}=10 / 1) ;{ }^{1} \mathbf{H}$ NMR $\left(400 \mathrm{MHz}, \mathrm{CDCl}_{3}\right): \delta 7.20(\mathrm{~d}, J=8.4 \mathrm{~Hz}, 1 \mathrm{H}) ; 6.70(\mathrm{dd}, J=8.8,2.8 \mathrm{~Hz}$, $1 \mathrm{H}), 6.64(\mathrm{~d}, J=2.4 \mathrm{~Hz}, 1 \mathrm{H}), 3.96(\mathrm{t}, J=6.0 \mathrm{~Hz}, 2 \mathrm{H}), 3.26(\mathrm{t}, J=6.8 \mathrm{~Hz}, 2 \mathrm{H}), 2.92-2.88(\mathrm{~m}, 2 \mathrm{H})$, $2.50(\mathrm{dd}, J=18.4,8.8 \mathrm{~Hz}, 1 \mathrm{H}), 2.42-2.37(\mathrm{~m}, 1 \mathrm{H}), 2.27-2.22(\mathrm{~m}, 1 \mathrm{H}), 2.19-1.98(\mathrm{~m}, 5 \mathrm{H}), 1.97-1.93$ (m, 1H), 1.92-1.85 (m, 2H), 1.68-1.38 (m, 6H), $0.91(\mathrm{~s}, 3 \mathrm{H}) ;{ }^{13} \mathbf{C} \mathbf{N M R}\left(100 \mathrm{MHz}, \mathrm{CDCl}_{3}\right): 220.1$, $156.8,137.7,132.0,126.3,114.4,112.0,66.4,50.3,47.9,43.9,38.3,35.8,31.5,30.1,29.6,26.5,25.9$, $21.5,13.8,6.5$. The spectral data are consistent with those reported in the literature. ${ }^{[19]}$

\section{Procedure for the synthesis of the alkyl iodide 10} $(3 R, 5 R, 6 S, 8 S, 9 S, 10 R, 13 R, 14 S, 17 S)-17-((R)-5$-iodopentan-2-yl)-3,6-dimethoxy-10,13dimethylhexadecahydro- $1 H$-cyclopenta[a]phenanthrene (10)

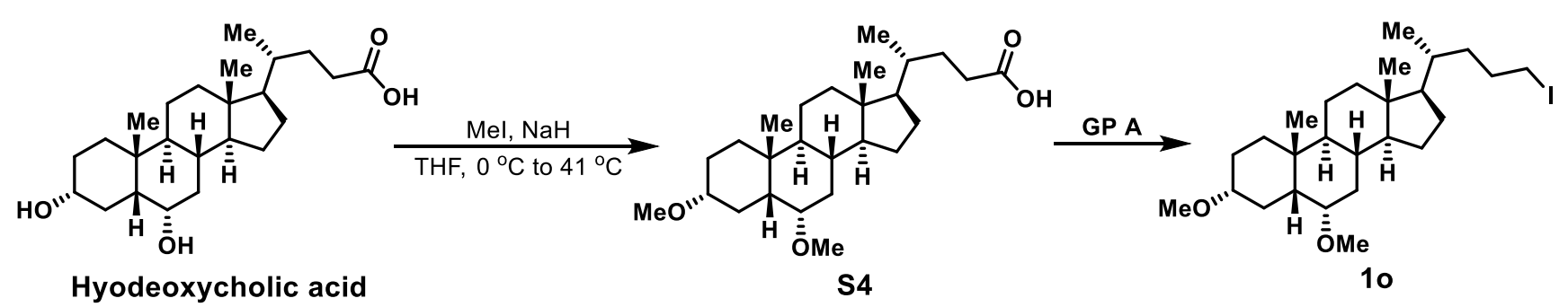

To a solution of hyodeoxycholic acid (1.0 equiv, $10 \mathrm{mmol}, 3.9 \mathrm{~g})$ in THF $(0.1 \mathrm{M}, 100 \mathrm{~mL})$ at $0{ }^{\circ} \mathrm{C}$ was added $\mathrm{NaH}$ (6.0 equiv, $60 \%$ dispersion in mineral oil, $60 \mathrm{mmol}, 2.4 \mathrm{~g}$ ) in small portions. The reaction mixture was allowed to stir for $1 \mathrm{~h}$ at room temperature, then it was cooled to $0{ }^{\circ} \mathrm{C}$. MeI $(10$ equiv, $100 \mathrm{mmol}, 6.4 \mathrm{~mL}$ ) was slowly added to the mixture and it was allowed to stir at $41{ }^{\circ} \mathrm{C}$ for 26 hours by TLC monitor. A second portion of $\mathrm{NaH}$ (6.0 equiv, $60 \%$ dispersion in mineral oil, $60 \mathrm{mmol}$, $2.4 \mathrm{~g}$ ) and $\mathrm{MeI}$ (4.7 equiv, $47 \mathrm{mmol}, 3.0 \mathrm{~mL}$ ) were added to the reaction mixture and the reaction was allowed to stir at $41{ }^{\circ} \mathrm{C}$ for by TLC monitor. A third portion of $\mathrm{NaH}$ (6.0 equiv, $60 \%$ dispersion in mineral oil, $60 \mathrm{mmol}, 2.4 \mathrm{~g}$ ) and $\mathrm{MeI}(4.7$ equiv, $47 \mathrm{mmol}, 3.0 \mathrm{~mL}$ ) were added to the reaction mixture and the reaction was allowed to stir at a $41{ }^{\circ} \mathrm{C}$ oil bath for another $24 \mathrm{~h}$. The reaction was quenched with sat. aq. $\mathrm{NH}_{4} \mathrm{Cl}$ at $0{ }^{\circ} \mathrm{C}$ and extracted three times with ethyl acetate The combined organic layer was washed with brine, dried over $\mathrm{Na}_{2} \mathrm{SO}_{4}$, filtered and concentrated in vacuo. The resulting residue was purified by flash column chromatography on silica gel afforded $\mathbf{S 4}$ as a yellow oil $(3.7 \mathrm{~g}, 88 \%)$, $\mathbf{R}_{\mathbf{f}}=0.65(\mathrm{PE} / \mathrm{EtOAc}=20 / 1)$.

General procedure A was followed on $1.25 \mathrm{mmol}$ and purification by flash column chromatography on silica gel afforded 10 as a yellow oil (over two steps, $336.5 \mathrm{mg}, 52 \%$ ). $\mathbf{R}_{\mathbf{f}}=0.76$ $(\mathrm{PE} / \mathrm{EtOAc}=5 / 1) ;{ }^{1} \mathbf{H}$ NMR $\left(400 \mathrm{MHz}, \mathrm{CDCl}_{3}\right): \delta 3.47(\mathrm{dt}, J=12.0,4.4 \mathrm{~Hz}, 1 \mathrm{H}), 3.31(\mathrm{~s}, 3 \mathrm{H}), 3.25$ $(\mathrm{s}, 3 \mathrm{H}), 3.17-3.05(\mathrm{~m}, 3 \mathrm{H}), 1.93-1.77(\mathrm{~m}, 5 \mathrm{H}), 1.75-1.62(\mathrm{~m}, 5 \mathrm{H}), 1.57-1.51(\mathrm{~m}, 1 \mathrm{H}), 1.47-1.34(\mathrm{~m}$, 
5H), 1.27-1.18 (m, 3H), 1.14-1.02 (m, 7H), $0.87(\mathrm{~d}, J=7.2 \mathrm{~Hz}, 3 \mathrm{H}), 0.86(\mathrm{~s}, 3 \mathrm{H}), 0.59(\mathrm{~s}, 3 \mathrm{H}) ;{ }^{13} \mathrm{C}$ NMR $\left(100 \mathrm{MHz}, \mathrm{CDCl}_{3}\right): \delta 80.1,77.1,56.2,56.0,55.7,55.6,44.9,42.8,40.0,39.9,36.8,36.0,35.7$, 35.1, 34.7, 31.7, 30.4, 28.3, 27.0, 25.5, 24.2, 23.7, 20.8, 18.7, 12.1, 7.9; HRMS (EI): Calcd for $\mathrm{C}_{26} \mathrm{H}_{45} \mathrm{IO}_{2}$ : 516.2464; found: 516.2462.

\section{Procedure for the synthesis of the alkyl iodide $1 p$}

(R)-6-(4-iodobutoxy)-2,5,7,8-tetramethyl-2-((4R,8R)-4,8,12-trimethyltridecyl) chromane (1p)
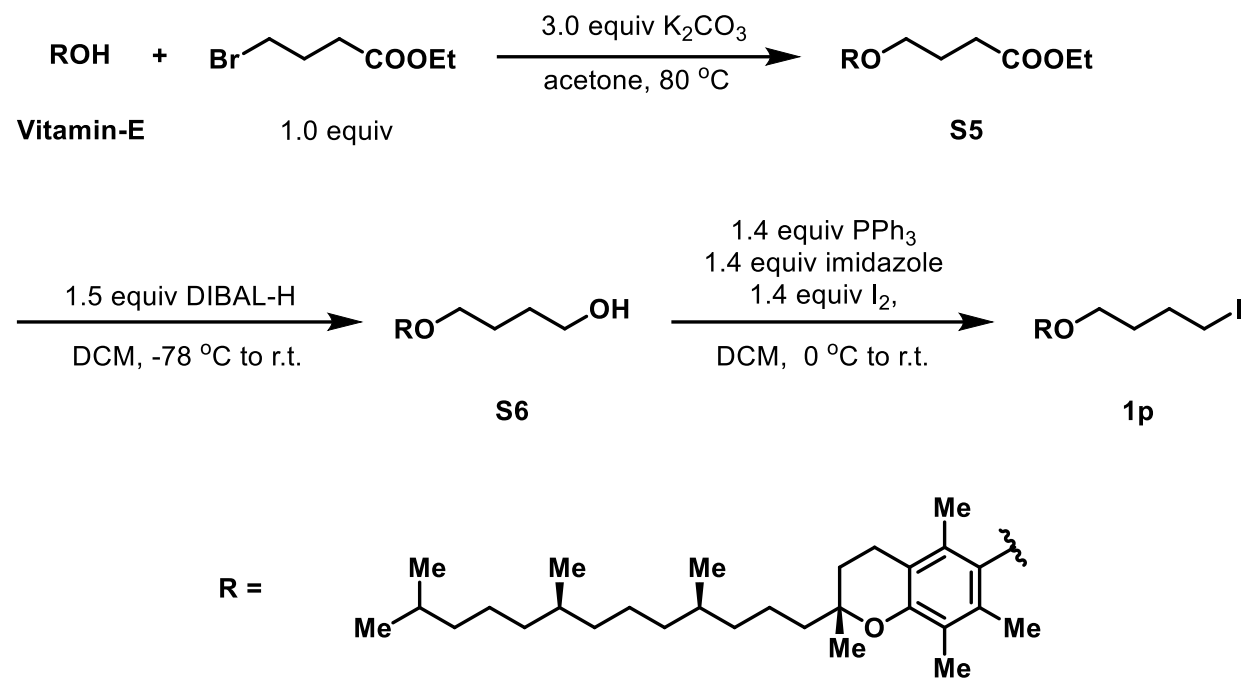

To a solution of $\mathrm{K}_{2} \mathrm{CO}_{3}(3.0$ equiv, $30 \mathrm{mmol}, 4.1 \mathrm{~g})$ in acetone $(0.5 \mathrm{M}, 20 \mathrm{~mL})$ was added VitaminE (1.0 equiv, $10 \mathrm{mmol}, 4.5 \mathrm{~mL}$ ) and ethyl 4-bromobutanoate (1.0 equiv, $10 \mathrm{mmol}, 1.4 \mathrm{~mL})$. The reaction was then allowed to stir at a $80{ }^{\circ} \mathrm{C}$ oil bath until complete by TLC analysis. The reaction was quenched with sat. aq. $\mathrm{NH}_{4} \mathrm{Cl}$ and extracted three times with ethyl acetate. The combined organic layer was washed with brine, dried over $\mathrm{Na}_{2} \mathrm{SO}_{4}$, filtered and concentrated in vacuo. The crude ester $\mathbf{S 5}$ was used in the next step without further purification.

To a solution of ester $\mathbf{S 5}$ (1.0 equiv) in DCM $(0.3 \mathrm{M}, 30 \mathrm{~mL})$ at $-78{ }^{\circ} \mathrm{C}$ was added DIBAL-H (1.5 $\mathrm{M}, 1.5$ equiv, $15 \mathrm{mmol}, 10 \mathrm{~mL}$ ) dropwise and the reaction mixture was stirred at this temperature overnight. The reaction mixture was quenched with $1.0 \mathrm{M}$ aq. $\mathrm{NaOH}$ and filtered through Celite. The aqueous layer was extracted three times with DCM and separated organic layer was washed with brine, dried over $\mathrm{Na}_{2} \mathrm{SO}_{4}$ and concentrated under reduced pressure. The resulting residue was purified by flash column chromatography on silica gel to afford S6 as a white solid $(1.1 \mathrm{~g}, 25 \%), \mathbf{R}_{\mathbf{f}}=0.44$ $(\mathrm{PE} / \mathrm{EtOAc}=5 / 2)$.

To a solution of $\mathrm{PPh}_{3}(1.4$ equiv, $3.2 \mathrm{mmol}, 844.6 \mathrm{mg}$ ) and imidazole (1.4 equiv, $3.2 \mathrm{mmol}, 217.8$ $\mathrm{mg})$ in $\operatorname{DCM}(0.2 \mathrm{M}, 10 \mathrm{~mL})$ at $0^{\circ} \mathrm{C}$ was added $\mathbf{S 6}(1.0$ equiv, $2.2 \mathrm{mmol}, 1.1 \mathrm{~g})$, and then $\mathrm{I}_{2}(1.4$ equiv, $3.2 \mathrm{mmol}, 812.2 \mathrm{mg}$ ) was added in small portions. The mixture was slowly warm to room temperature. The reaction mixture was stirred at room temperature until complete by TLC analysis. When the starting material was consumed, the reaction was quenched with the addition of sat. aq. $\mathrm{Na}_{2} \mathrm{~S}_{2} \mathrm{O}_{3}$ and extracted three times with DCM. The combined organic layer was dried over $\mathrm{Na}_{2} \mathrm{SO}_{4}$, and then filtered. The filtrate was concentrated in vacuo and the residue was purified by flash column chromatography on silica gel to afford $\mathbf{1 p}$ as a yellow oil $(1.0 \mathrm{~g}, 80 \%), \mathbf{R}_{\mathbf{f}}=0.35(\mathrm{PE} / \mathrm{EtOAc}=100 / 1) ;{ }^{1} \mathbf{H} \mathbf{~ N M R}(400$ $\left.\mathrm{MHz} \mathrm{CDCl}_{3}\right): \delta 3.56(\mathrm{t}, J=5.6 \mathrm{~Hz}, 2 \mathrm{H}), 3.20(\mathrm{t}, J=7.2 \mathrm{~Hz}, 2 \mathrm{H}), 2.47(\mathrm{t}, J=6.8 \mathrm{~Hz}, 2 \mathrm{H}), 2.06(\mathrm{~s}, 3 \mathrm{H})$, $2.02(\mathrm{~s}, 3 \mathrm{H}), 2.00(\mathrm{~s}, 3 \mathrm{H}), 1.80$ (quin, $J=6.8 \mathrm{~Hz}, 2 \mathrm{H}), 1.74-1.62(\mathrm{~m}, 2 \mathrm{H}), 1.52-1.40(\mathrm{~m}, 3 \mathrm{H}), 1.37-$ $1.14(\mathrm{~m}, 16 \mathrm{H}), 1.07-0.92(\mathrm{~m}, 7 \mathrm{H}), 0.79-0.75(\mathrm{~m}, 12 \mathrm{H}) ;{ }^{13} \mathbf{C}$ NMR (100 MHz, $\left.\mathrm{CDCl}_{3}\right): \delta 148.3,147.8$, 
127.7, 125.7, 122.9, 117.5, 74.8, 71.7, 40.2, 39.5, 37.7, 37.6, 37.5, 37.4, 32.9, 32.8, 31.4, 30.6, 28.1, 24.9, 24.6, 24.0, 22.9, 22.8, 21.1, 20.8, 19.9, 19.8, 19.7, 12.9, 12.0, 11.9, 6.7; HRMS (ESI): Calcd for $\mathrm{C}_{33} \mathrm{H}_{58} \mathrm{IO}_{2}[\mathrm{M}+\mathrm{H}]^{+}:$613.3476; found: 613.3473 .

\section{(S)-2-(1-iodopropan-2-yl)-6-methoxynaphthalene (1k)}

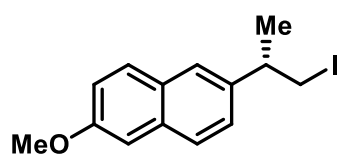

General procedure A was followed and purification by flash column chromatography on silica gel afforded $1 \mathbf{k}$ from Naproxen as a yellow solid (over two steps, $83 \%)$. $\mathbf{R}_{\mathbf{f}}=0.48(\mathrm{PE}) ;{ }^{1} \mathbf{H}$ NMR $\left(400 \mathrm{MHz}, \mathrm{CDCl}_{3}\right): \delta 7.57(\mathrm{~d}, J=8.8$ $\mathrm{Hz}, 2 \mathrm{H}), 7.43(\mathrm{~d}, J=1.6 \mathrm{~Hz}, 1 \mathrm{H}), 7.15(\mathrm{dd}, J=8.4,1.6 \mathrm{~Hz}, 1 \mathrm{H}), 7.03$ (dd, $J=$ 8.8, $2.4 \mathrm{~Hz}, 1 \mathrm{H}), 6.99(\mathrm{~d}, J=2.4 \mathrm{~Hz}, 1 \mathrm{H}), 3.76(\mathrm{~s}, 3 \mathrm{H}), 3.33(\mathrm{dd}, J=9.6,6.0 \mathrm{~Hz}, 1 \mathrm{H}), 3.24(\mathrm{dd}, J=$ 9.6, $8.0 \mathrm{~Hz}, 1 \mathrm{H}), 3.03$ (sext, $J=6.8 \mathrm{~Hz}, 1 \mathrm{H}), 1.36$ (d, $J=7.2 \mathrm{~Hz}, 3 \mathrm{H}) ;{ }^{13} \mathbf{C} \mathbf{N M R}\left(100 \mathrm{MHz}, \mathrm{CDCl}_{3}\right)$ : $\delta 157.6,139.5,133.7,129.3,129.0,127.2,125.7,125.2,119.0,105.7,55.4,42.3,21.7,15.3$; HRMS (EI): Calcd for $\mathrm{C}_{14} \mathrm{H}_{15} \mathrm{IO}$ : 326.0168 ; found: 326.0164 .

\section{Synthesis and Characterization of New Products}

\section{General Procedure for the Aminocarbonylation of Alkyl Halides}

\section{General procedure A for Ni-catalyzed aminocarbonylation of primary alkyl iodides.}

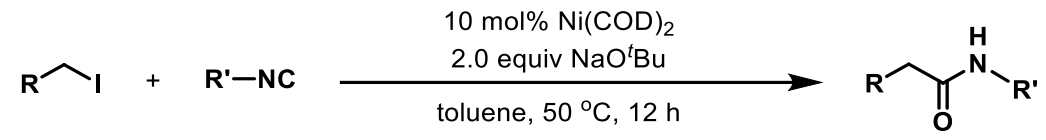

To a solution of $\mathrm{Ni}(\mathrm{COD})_{2}(10 \mathrm{~mol} \%)$ and sodium tert-butoxide $(2.0$ equiv $)$ in toluene $(0.1 \mathrm{M})$ was added tert-butyl isocyanide (1.5 equiv) and alkyl iodide (1.0 equiv) in turn. The reaction mixture was stirred at a $50{ }^{\circ} \mathrm{C}$ oil bath until complete by TLC analysis. When the starting material was consumed, the reaction was quenched with $1 \mathrm{M}$ aq. $\mathrm{HCl}$ and extracted three times with ethyl acetate. The combined organic layer was dried over $\mathrm{Na}_{2} \mathrm{SO}_{4}$, and then filtered. The filtrate was concentrated in vacuo and the residue was purified by flash column chromatography.

\section{General procedure B for Ni-catalyzed aminocarbonylation of secondary alkyl iodides.}

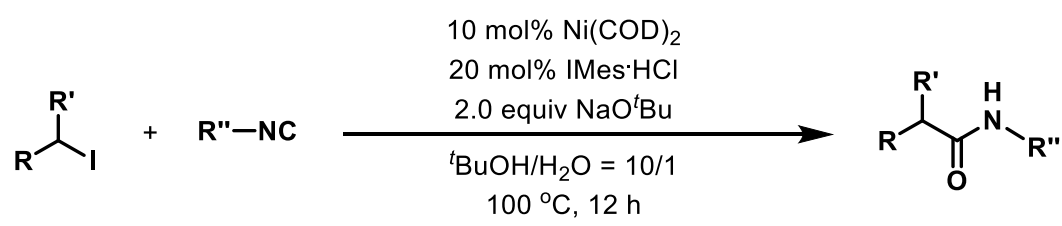

To a solution of $\mathrm{Ni}(\mathrm{COD})_{2}(10 \mathrm{~mol} \%)$, IMes $\bullet \mathrm{HCl}(20 \mathrm{~mol} \%)$ and sodium tert-butoxide (2.0 equiv) in tert-butanol $/ \mathrm{H}_{2} \mathrm{O}(10 / 1 ; 0.1 \mathrm{M})$ was added tert-butyl isocyanide (1.5 equiv) and alkyl iodide (1.0 equiv) in turn. The reaction mixture was stirred at a $100{ }^{\circ} \mathrm{C}$ oil bath until complete by TLC analysis. When the starting material was consumed, the reaction was quenched with $1 \mathrm{M}$ aq. $\mathrm{HCl}$ and extracted three times with ethyl acetate. The combined organic layer was dried over $\mathrm{Na}_{2} \mathrm{SO}_{4}$, and then filtered. 
The filtrate was concentrated in vacuo and the residue was purified by flash column chromatography.

\section{Procedure for the Synthesis of the amide 3a on a 1.0 mmol-scale}

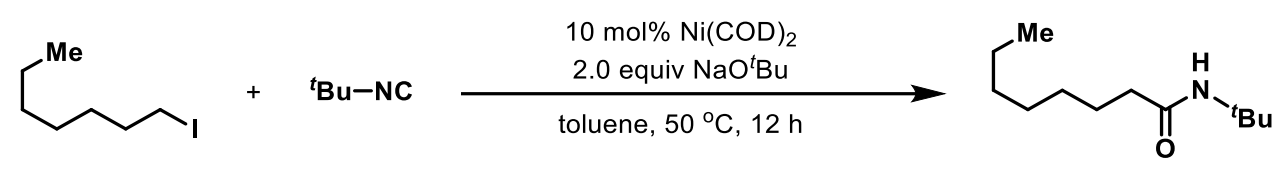

To a solution of $\mathrm{Ni}(\mathrm{COD})_{2}(10 \mathrm{~mol} \%, 0.1 \mathrm{mmol}, 27.5 \mathrm{mg})$ and sodium tert-butoxide (2.0 equiv, $2.0 \mathrm{mmol}, 192.2 \mathrm{mg})$ in toluene $(0.1 \mathrm{M}, 10 \mathrm{~mL})$ was added tert-butyl isocyanide (1.5 equiv, $1.5 \mathrm{mmol}$, $0.17 \mathrm{~mL}$ ) and alkyl iodide (1.0 equiv, $1.0 \mathrm{mmol}, 0.16 \mathrm{~mL})$ in turn. The reaction mixture was stirred at a $50{ }^{\circ} \mathrm{C}$ oil bath until complete by TLC analysis. When the starting material was consumed, the reaction was quenched with $1 \mathrm{M}$ aq. $\mathrm{HCl}$ and extracted three times with ethyl acetate. The combined organic layer was dried over $\mathrm{Na}_{2} \mathrm{SO}_{4}$, and then filtered. The filtrate was concentrated in vacuo and the residue was purified by flash column chromatography $(\mathrm{PE} / \mathrm{EtOAc}=5 / 1)$ to afforded 3a as a yellow oil (199 $\mathrm{mg}, 99 \%) . \mathbf{R} \mathbf{f}=0.37(\mathrm{PE} / \mathrm{EtOAc}=5 / 1) ;{ }^{1} \mathbf{H}$ NMR $\left(400 \mathrm{MHz}, \mathrm{CDCl}_{3}\right): \delta 5.28(\mathrm{bs}, 1 \mathrm{H}), 2.07(\mathrm{t}, J=7.6$ $\mathrm{Hz}, 2 \mathrm{H}), 1.58$ (quin, $J=7.2 \mathrm{~Hz}, 2 \mathrm{H}), 1.33(\mathrm{~s}, 9 \mathrm{H}), 1.28-1.25(\mathrm{~m}, 8 \mathrm{H}), 0.86(\mathrm{t}, J=6.8 \mathrm{~Hz}, 3 \mathrm{H}) ;{ }^{13} \mathrm{C}$ NMR (100 MHz, $\left.\mathrm{CDCl}_{3}\right): \delta 172.7,51.2,37.9,31.8,29.3,29.2,29.0,25.9,22.7,14.2$. The spectral data are consistent with those reported in the literature. ${ }^{[20]}$

\section{Characterization of New Products}

\section{$N$-(tert-butyl)-4-phenylbutanamide (3b)}<smiles>O=C(CCCc1ccccc1)NC(=O)CCc1ccccc1</smiles>

General procedure A was followed on $0.3 \mathrm{mmol}$ scale and purification by flash column chromatography on silica gel $(\mathrm{PE} / \mathrm{EtOAc}=5 / 1)$ afforded $\mathbf{3 b}$ as a yellow oil $(50.0 \mathrm{mg}, 76 \%) . \mathbf{R}_{\mathbf{f}}=0.42(\mathrm{PE} / \mathrm{EtOAc}=5 / 1) ;{ }^{1} \mathbf{H} \mathbf{~ N M R}\left(400 \mathrm{MHz}, \mathrm{CDCl}_{3}\right)$ : $\delta 7.22-7.18(\mathrm{~m}, 2 \mathrm{H}), 7.12-7.09(\mathrm{~m}, 3 \mathrm{H}), 5.24(\mathrm{bs}, 1 \mathrm{H}), 2.56(\mathrm{t}, J=7.2 \mathrm{~Hz}, 2 \mathrm{H}), 2.01$ (t, $J=7.2 \mathrm{~Hz}$, $2 \mathrm{H}), 1.90-1.83(\mathrm{~m}, 2 \mathrm{H}), 1.26(\mathrm{~s}, 9 \mathrm{H}) ;{ }^{13} \mathbf{C} \mathbf{N M R}\left(100 \mathrm{MHz}, \mathrm{CDCl}_{3}\right): \delta 172.1,141.7,128.6,128.4$, $126.0,51.2,36.8,35.2,28.9,27.2$. The spectral data are consistent with those reported in the literature. ${ }^{[21]}$

\section{$N$-(tert-butyl)-4,8-dimethylnon-7-enamide (3c)}

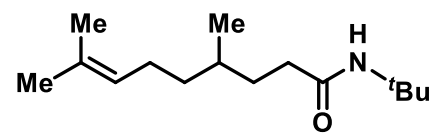

General procedure A was followed on $0.3 \mathrm{mmol}$ scale and purification by flash column chromatography on silica gel $(\mathrm{PE} /$ EtOAc $=5 / 1)$ afforded $3 \mathbf{c}$ as a yellow oil $(63.1 \mathrm{mg}, 89 \%) . \mathbf{R}_{\mathbf{f}}=0.50(\mathrm{PE} /$ EtOAc $=5 / 1) ;{ }^{1} \mathbf{H} \mathbf{~ N M R}$ $\left(400 \mathrm{MHz}, \mathrm{CDCl}_{3}\right): \delta 5.33(\mathrm{bs}, 1 \mathrm{H}), 5.05(\mathrm{t}, J=7.2 \mathrm{~Hz}, 1 \mathrm{H}), 2.13-1.98(\mathrm{~m}, 2 \mathrm{H}), 1.96-1.86(\mathrm{~m}, 2 \mathrm{H})$, $1.64(\mathrm{~s}, 3 \mathrm{H}), 1.62-1.59(\mathrm{~m}, 1 \mathrm{H}), 1.56(\mathrm{~s}, 3 \mathrm{H}), 1.42-1.37(\mathrm{~m}, 2 \mathrm{H}), 1.31(\mathrm{~s}, 9 \mathrm{H}), 1.27-1.22(\mathrm{~m}, 1 \mathrm{H})$, $1.16-1.07(\mathrm{~m}, 1 \mathrm{H}), 0.85(\mathrm{~d}, J=6.4 \mathrm{~Hz}, 3 \mathrm{H}) ;{ }^{13} \mathbf{C} \mathbf{N M R}\left(100 \mathrm{MHz}, \mathrm{CDCl}_{3}\right): \delta 172.8,131.2,124.8,51.0$, 36.9, 35.4, 32.8, 32.2 , 28.9, 25.8, 25.5, 19.4, 17.7; HRMS (EI): Calcd for $\mathrm{C}_{15} \mathrm{H}_{29} \mathrm{NO}$ : 239.2249; found: 239.2246 . 


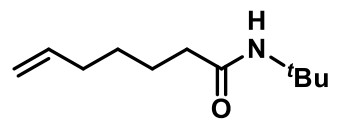

General procedure A was followed on $0.3 \mathrm{mmol}$ scale and purification by flash column chromatography on silica gel $(\mathrm{PE} / \mathrm{EtOAc}=5 / 1)$ afforded $\mathbf{3 d}$ as a yellow oil $(39.3 \mathrm{mg}, 72 \%)$. When the 6-bromohex-1-ene was utilized as the starting mate-rial, the isolated yield of amide 3d was 70\%. $\mathbf{R}_{\mathbf{f}}=0.43(\mathrm{PE} / \mathrm{EtOAc}=5 / 1) ;{ }^{1} \mathbf{H} \mathbf{~ N M R}(400 \mathrm{MHz}$, $\left.\mathrm{CDCl}_{3}\right): \delta 5.75$ (ddt, $\left.J=16.8,10.4,6.8 \mathrm{~Hz}, 1 \mathrm{H}\right), 5.35(\mathrm{bs}, 1 \mathrm{H}), 4.97(\mathrm{dd}, J=17.2,1.6 \mathrm{~Hz}, 1 \mathrm{H}), 4.91$ (d, $J=10.0 \mathrm{~Hz}, 1 \mathrm{H}), 2.08-2.08(\mathrm{~m}, 4 \mathrm{H}), 1.62-1.55(\mathrm{~m}, 2 \mathrm{H}), 1.41-1.35(\mathrm{~m}, 2 \mathrm{H}), 1.31(\mathrm{~s}, 9 \mathrm{H}) ;{ }^{13} \mathrm{C}$ NMR (100 MHz, $\left.\mathrm{CDCl}_{3}\right): \delta 172.5,138.7,114.7,51.1,37.6,33.6,28.9,28.5,25.3$. The spectral data are consistent with those reported in the literature. ${ }^{[22]}$

\section{$N$-(tert-butyl)-7-phenylhept-6-ynamide (3e)}

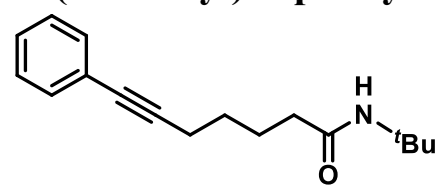

General procedure A was followed on $0.3 \mathrm{mmol}$ scale and purification by flash column chromatography on silica gel $(\mathrm{PE} / \mathrm{EtOAc}=5 / 1)$ afforded $\mathbf{3 e}$ as a yellow solid $(51.7 \mathrm{mg}, 67 \%) . \mathbf{R}_{\mathbf{f}}=0.14(\mathrm{PE} / \mathrm{EtOAc}=5 / 1) ;{ }^{1} \mathbf{H} \mathbf{~ N M R}$ $\left(400 \mathrm{MHz}, \mathrm{CDCl}_{3}\right): \delta 7.32-7.30(\mathrm{~m}, 2 \mathrm{H}), 7.20-7.18(\mathrm{~m}, 3 \mathrm{H}), 5.29(\mathrm{bs}, 1 \mathrm{H})$, 2.35 (t, $J=7.2 \mathrm{~Hz}, 2 \mathrm{H}$ ), 2.07 (t, $J=7.2 \mathrm{~Hz}, 2 \mathrm{H}$ ), 1.70 (quin, $J=7.2 \mathrm{~Hz}, 2 \mathrm{H}$ ), 1.55 (quin, $J=6.8 \mathrm{~Hz}$, 2H), 1.26 (s, 9H); ${ }^{13} \mathbf{C}$ NMR (100 MHz, $\left.\mathrm{CDCl}_{3}\right): \delta 172.2,131.6,128.3,127.7,124.0,90.0,81.0,51.2$, 37.2, 28.9, 28.3, 25.1, 19.3; HRMS (EI): Calcd for $\mathrm{C}_{17} \mathrm{H}_{23} \mathrm{NO}$ : 257.1780; found: 257.1776.

\section{$N$-(tert-butyl)-4-((tert-butyldimethylsilyl)oxy)butanamide (3f)}

$\mathrm{N}_{\mathrm{N}}$
$\mathrm{t}_{\mathrm{Bu}}$ column chromatography on silica gel $(\mathrm{PE} / \mathrm{EtOAc}=10 / 1)$ afforded $\mathbf{3 f}$ as a yellow solid $(80.3 \mathrm{mg}, 98 \%) . \mathbf{R}_{\mathbf{f}}=0.52(\mathrm{PE} / \mathrm{EtOAc}=10 / 1) ;{ }^{1} \mathbf{H}$ NMR $(400$

$\left.\mathrm{MHz}, \mathrm{CDCl}_{3}\right): \delta 5.45(\mathrm{bs}, 1 \mathrm{H}), 3.59(\mathrm{t}, J=6.0 \mathrm{~Hz}, 2 \mathrm{H}), 2.14(\mathrm{t}, J=7.2 \mathrm{~Hz}, 2 \mathrm{H}), 1.80-1.73(\mathrm{~m}, 2 \mathrm{H})$, $1.30(\mathrm{~s}, 9 \mathrm{H}), 0.85$ (s, 9H), 0.01 (s, $6 \mathrm{H}) ;{ }^{13} \mathbf{C}$ NMR $\left(100 \mathrm{MHz}, \mathrm{CDCl}_{3}\right): \delta 172.3,62.1,51.1,33.9,28.9$, 28.7, 26.0, 18.4, 5.2; HRMS (EI): Calcd for $\mathrm{C}_{14} \mathrm{H}_{31} \mathrm{NO}_{2} \mathrm{Si}$ : 273.2124; found: 273.2125 .

\section{$N$-(tert-butyl)-7-hydroxyheptanamide (3g)}<smiles>C[13CH2]NC(=O)CCCCO</smiles>

General procedure A was followed on $0.3 \mathrm{mmol}$ scale and purification by flash column chromatography on silica gel $(\mathrm{PE} / \mathrm{EtOAc}=1 / 1)$ afforded $\mathbf{3 g}$ as a yellow oil (25.5 mg, 42\%). $\mathbf{R}_{\mathbf{f}}=0.19(\mathrm{PE} / \mathrm{EtOAc}=1 / 1) ;{ }^{1} \mathbf{H} \mathbf{~ N M R}\left(400 \mathrm{MHz}, \mathrm{CDCl}_{3}\right): \delta$ $5.43(\mathrm{bs}, 1 \mathrm{H}), 3.64(\mathrm{t}, J=6.4 \mathrm{~Hz}, 2 \mathrm{H}), 2.13(\mathrm{t}, J=6.8 \mathrm{~Hz}, 2 \mathrm{H}), 1.89(\mathrm{bs}, 1 \mathrm{H}), 1.64-1.54(\mathrm{~m}, 4 \mathrm{H}), 1.42$ 1.31 (m, 13H); ${ }^{13} \mathbf{C}$ NMR $\left(100 \mathrm{MHz}, \mathrm{CDCl}_{3}\right): \delta 172.7,62.8,51.2,37.6,32.6,28.9,25.8,25.5$; HRMS (ESI): Calcd for $\mathrm{C}_{11} \mathrm{H}_{23} \mathrm{NO}_{2} \mathrm{Na}[\mathrm{M}+\mathrm{Na}]^{+}:$224.1626; found: 224.1621 .

\section{$N$-(tert-butyl)-6-(4,4,5,5-tetramethyl-1,3,2-dioxaborolan-2-yl)hexanamide (3h)}

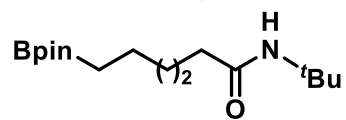

General procedure A was followed on $0.3 \mathrm{mmol}$ scale and purification by flash column chromatography on silica gel $(\mathrm{PE} / \mathrm{EtOAc}=5 / 1)$ afforded $\mathbf{3 h}$ as a yellow oil (43.2 mg, 48\%). $\mathbf{R}_{\mathbf{f}}=0.21(\mathrm{PE} / \mathrm{EtOAc}=5 / 1) ;{ }^{1} \mathbf{H} \mathbf{~ N M R}\left(400 \mathrm{MHz}, \mathrm{CDCl}_{3}\right)$ : $\delta 5.33$ (bs, $1 \mathrm{H}), 2.04$ (t, $J=7.6 \mathrm{~Hz}, 2 \mathrm{H}), 1.56$ (quin, $J=7.6 \mathrm{~Hz}, 2 \mathrm{H}), 1.42-1.34(\mathrm{~m}, 2 \mathrm{H}), 1.30$ (s, 9H), $1.27-1.22(\mathrm{~m}, 2 \mathrm{H}), 1.20(\mathrm{~s}, 12 \mathrm{H}), 0.73(\mathrm{t}, J=7.6 \mathrm{~Hz}, 2 \mathrm{H}) ;{ }^{13} \mathbf{C} \mathbf{~ N M R}\left(100 \mathrm{MHz}, \mathrm{CDCl}_{3}\right): \delta 172.7,83.0$, 51.1, 37.7, 32.0, 28.9, 25.7, 24. 9, 23.8; HRMS (EI): Calcd for $\mathrm{C}_{16} \mathrm{H}_{32} \mathrm{BNO}_{3}$ : 297.2475; found: 297.2478. 


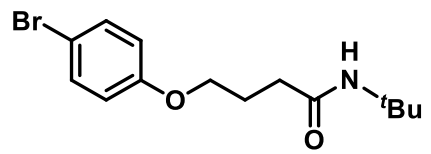

General procedure A was followed on $0.3 \mathrm{mmol}$ scale and purification by flash column chromatography on silica gel $(\mathrm{PE} / \mathrm{EtOAc}=5 / 1)$ afforded $\mathbf{3 i}$ as a yellow solid $(78.7 \mathrm{mg}, 84 \%) . \mathbf{R}_{\mathbf{f}}=0.19(\mathrm{PE} / \mathrm{EtOAc}=5 / 1) ;{ }^{1} \mathbf{H} \mathbf{~ N M R}$ (400 MHz, $\left.\mathrm{CDCl}_{3}\right): \delta 7.35-7.31(\mathrm{~m}, 2 \mathrm{H}), 6.76-6.72(\mathrm{~m}, 2 \mathrm{H}), 5.42(\mathrm{bs}, 1 \mathrm{H})$, $3.93(\mathrm{t}, J=6.0 \mathrm{~Hz}, 2 \mathrm{H}), 2.62(\mathrm{t}, J=7.2 \mathrm{~Hz}, 2 \mathrm{H}), 2.09-2.02(\mathrm{~m}, 2 \mathrm{H}), 1.30(\mathrm{~s}, 9 \mathrm{H}) ;{ }^{13} \mathbf{C} \mathbf{N M R}(100 \mathrm{MHz}$, $\left.\mathrm{CDCl}_{3}\right): \delta 171.5,158.1,132.3,116.3,112.8,67.2,51.2,33.7,28.9,25.2$. The spectral data are consistent with those reported in the literature.

\section{$N$-(tert-butyl)-7-(4-iodophenoxy)heptanamide (3j)}<smiles>O=C(CCOc1ccc(I)cc1)NC(=O)c1ccccc1</smiles>

General procedure A was followed on $0.3 \mathrm{mmol}$ scale and purification by flash column chromatography on silica gel $(\mathrm{PE} / \mathrm{EtOAc}=5 / 1)$ afforded $\mathbf{3} \mathbf{j}$ as a white solid (95.5 mg, 81\%). $\mathbf{R}=0.24(\mathrm{PE} / \mathrm{EtOAc}=5 / 1) ;{ }^{1} \mathbf{H}$ NMR $(400 \mathrm{MHz}$, $\left.\mathrm{CDCl}_{3}\right): \delta 7.52-7.49(\mathrm{~m}, 2 \mathrm{H}), 6.65-6.62(\mathrm{~m}, 2 \mathrm{H}), 5.32(\mathrm{bs}, 1 \mathrm{H}), 3.87(\mathrm{t}, J=6.4 \mathrm{~Hz}, 2 \mathrm{H}), 2.07(\mathrm{t}, J=$ $7.2 \mathrm{~Hz}, 2 \mathrm{H}), 1.78-1.71(\mathrm{~m}, 2 \mathrm{H}), 1.61$ (quin, $J=7.6 \mathrm{~Hz}, 2 \mathrm{H}), 1.48-1.40(\mathrm{~m}, 2 \mathrm{H}), 1.38-1.33(\mathrm{~m}, 2 \mathrm{H})$, $1.32(\mathrm{~s}, 9 \mathrm{H}) ;{ }^{13} \mathbf{C}$ NMR $\left(100 \mathrm{MHz}, \mathrm{CDCl}_{3}\right): \delta 172.4,159.0,138.2,117.0,82.5,68.0,51.1,37.6,29.0$, 29.0, 28.9, 25.8, 25.7; HRMS (EI): Calcd for $\mathrm{C}_{17} \mathrm{H}_{26} \mathrm{INO}_{2}$ : 403.1008; found: 403.0998 .

\section{(R)-N-(tert-butyl)-3-(6-methoxynaphthalen-2-yl)butanamide (3k)}

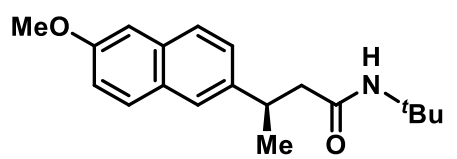

General procedure A was followed on $0.3 \mathrm{mmol}$ scale and purification by flash column chromatography on silica gel $(\mathrm{PE} / \mathrm{EtOAc}=5 / 1)$ afforded $\mathbf{3 k}$ as a light yellow solid $(55.6 \mathrm{mg}, 62 \%) . \mathbf{R}_{\mathbf{f}}=0.21(\mathrm{PE} / \mathrm{EtOAc}=5 / 1) ;{ }^{1} \mathbf{H}$ NMR $\left(400 \mathrm{MHz}, \mathrm{CDCl}_{3}\right): \delta 7.68(\mathrm{t}, J=8.4 \mathrm{~Hz}, 2 \mathrm{H}), 7.57(\mathrm{~d}, J=0.8 \mathrm{~Hz}$, $1 \mathrm{H}), 7.34(\mathrm{dd}, J=8.4,1.6 \mathrm{~Hz}, 1 \mathrm{H}), 7.14-7.10(\mathrm{~m}, 2 \mathrm{H}), 5.11(\mathrm{bs}, 1 \mathrm{H}), 3.90(\mathrm{~s}, 3 \mathrm{H}), 3.40$ (sext, $J=7.2$ $\mathrm{Hz}, 1 \mathrm{H}), 2.44-2.35$ (m, 2H), 1.38 (d, $J=7.2 \mathrm{~Hz}, 3 \mathrm{H}), 1.16$ (s, 9H); ${ }^{13} \mathbf{C}$ NMR $\left(100 \mathrm{MHz}, \mathrm{CDCl}_{3}\right): \delta$ 171.3, 157.4, 141.1, 133.4, 129.2, 129.1, 127.2, 126.0, 125.2, 118.9, 105.7, 55.4, 51.2, 47.1, 37.2, 28.8, 28.7, 21.6; HRMS (EI): Calcd for $\mathrm{C}_{19} \mathrm{H}_{25} \mathrm{NO}_{2}: 299.1885$; found: 299.1884 .

\section{$N$-((3s,5s,7s)-adamantan-1-yl)octanamide (3l)}

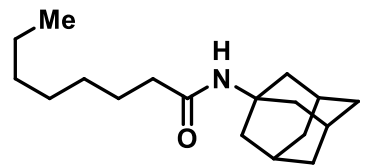

General procedure A was followed on $0.3 \mathrm{mmol}$ scale and purification by flash column chromatography on silica gel $(\mathrm{PE} / \mathrm{EtOAc}=20 / 1)$ afforded $3 \mathbf{I}$ as a yellow solid $(83.5 \mathrm{mg}, 99 \%) . \mathbf{R} \mathbf{f}=0.19(\mathrm{PE} / \mathrm{EtOAc}=20 / 1) ;{ }^{1} \mathbf{H}$ NMR $(400$ $\left.\mathrm{MHz}, \mathrm{CDCl}_{3}\right): \delta 5.25(\mathrm{bs}, 1 \mathrm{H}), 2.06-2.02(\mathrm{~m}, 5 \mathrm{H}), 1.96(\mathrm{~d}, J=2.8 \mathrm{~Hz}, 6 \mathrm{H})$, $1.63(\mathrm{t}, J=3.2 \mathrm{~Hz}, 6 \mathrm{H}), 1.55(\mathrm{t}, J=7.2 \mathrm{~Hz}, 2 \mathrm{H}), 1.27-1.22(\mathrm{~m}, 8 \mathrm{H}), 0.84(\mathrm{t}, J=6.8 \mathrm{~Hz}, 3 \mathrm{H}) ;{ }^{13} \mathbf{C}$ NMR $\left(100 \mathrm{MHz}, \mathrm{CDCl}_{3}\right): \delta 172.5,51.8,41.7,37.8,36.4,31.8,29.5,29.2,29.1,25.9,22.7,14.1$. The spectral data are consistent with those reported in the literature. ${ }^{[23]}$

\section{$\mathrm{N}$-(2,4,4-trimethylpentan-2-yl)octanamide (3m)}

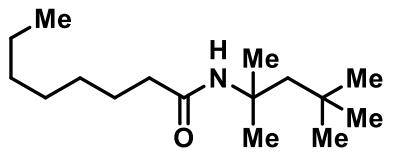

General procedure A was followed on $0.1 \mathrm{mmol}$ scale and purification by flash column chromatography on silica gel $(\mathrm{PE} / \mathrm{EtOAc}=5 / 1)$ afforded $3 \mathrm{~m}$ as a yellow oil $(23.3 \mathrm{mg}, 91 \%) . \mathbf{R} \mathbf{f}=0.52(\mathrm{PE} / \mathrm{EtOAc}=5 / 1) ;{ }^{1} \mathbf{H}$ NMR $(400$ $\left.\mathrm{MHz}, \mathrm{CDCl}_{3}\right): \delta 5.28(\mathrm{bs}, 1 \mathrm{H}), 2.06(\mathrm{t}, J=7.2 \mathrm{~Hz}, 2 \mathrm{H}), 1.73(\mathrm{~s}, 2 \mathrm{H}), 1.61-1.54(\mathrm{~m}, 2 \mathrm{H}), 1.38(\mathrm{~s}, 6 \mathrm{H})$, $1.32-1.26(\mathrm{~m}, 8 \mathrm{H}), 0.99(\mathrm{~s}, 9 \mathrm{H}), 0.89-0.83(\mathrm{~m}, 3 \mathrm{H}) ;{ }^{13} \mathbf{C}$ NMR $\left(100 \mathrm{MHz}, \mathrm{CDCl}_{3}\right): \delta 172.3,55.2,51.7$, $38.1,31.8,31.8,31.6,29.4,29.4,29.2,25.7,22.7,14.2$. The spectral data are consistent with those reported in the literature. ${ }^{[24]}$ 
$N$-(tert-butyl)-5-(((8R,9S,13S,14S)-13-methyl-17-oxo-7,8,9,11,12,13,14,15,16,17-decahydro-6Hcyclopenta[a]phenanthren-3-yl)oxy)pentanamide (3n)

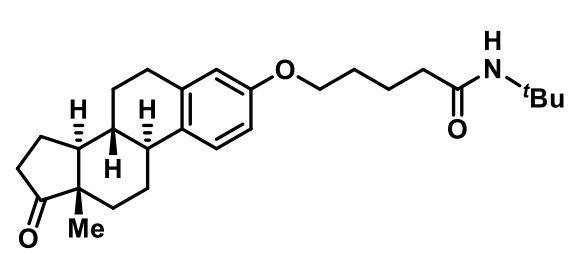

General procedure A was followed on $0.3 \mathrm{mmol}$ scale and purification by flash column chromatography on silica gel $(\mathrm{PE} / \mathrm{EtOAc}=1 / 1)$ afforded $\mathbf{3 n}$ as a white solid $(60.2 \mathrm{mg}, 47 \%)$. $\mathbf{R}_{\mathbf{f}}$ $=0.48(\mathrm{PE} / \mathrm{EtOAc}=1 / 1) ;{ }^{1} \mathbf{H}$ NMR $\left(400 \mathrm{MHz}, \mathrm{CDCl}_{3}\right): \delta 7.17(\mathrm{~d}$, $J=8.4 \mathrm{~Hz}, 1 \mathrm{H}), 6.69(\mathrm{dd}, J=8.4,2.8 \mathrm{~Hz}, 1 \mathrm{H}), 6.62(\mathrm{~d}, J=2.4 \mathrm{~Hz}$, 1H), 5.45 (bs, 1H), 3.94 (t, $J=5,2 \mathrm{~Hz}, 2 \mathrm{H}), 2.90-2.86$ (m, 2H), 2.49 (dd, $J=18.8,8.8 \mathrm{~Hz}, 1 \mathrm{H}), 2.40$ $2.36(\mathrm{~m}, 1 \mathrm{H}), 2.25-1.92(\mathrm{~m}, 7 \mathrm{H}), 1.80-1.76(\mathrm{~m}, 4 \mathrm{H}), 1.66-1.39(\mathrm{~m}, 6 \mathrm{H}), 1.34(\mathrm{~s}, 9 \mathrm{H}), 0.89(\mathrm{~s}, 3 \mathrm{H})$; ${ }^{13} \mathrm{C}$ NMR $\left(100 \mathrm{MHz}, \mathrm{CDCl}_{3}\right): \delta 221.1,172.2,157.0,137.8,132.0,126.4,114.5,112.1,67.6,51.2$, 50.4, 48.1, 44.0, 38.4, 37.2, 35.9, 31.6, 29.7, 28.9, 28.7, 26.6, 26.0, 22.6, 21.6, 13.9; HRMS (EI): Calcd for $\mathrm{C}_{27} \mathrm{H}_{39} \mathrm{NO}_{3}$ : 425.2930; found: 425.2931 .

trimethylhexadecahydro-1H-cyclopenta[a]phenanthren-17-yl)hexanamide (3o)

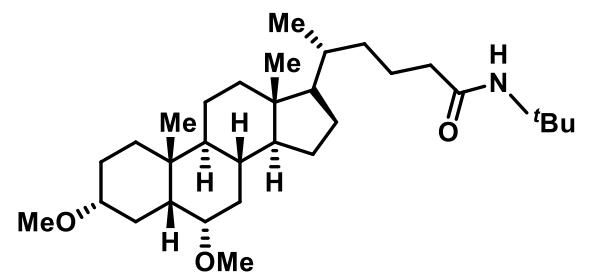

General procedure A was followed on $0.2 \mathrm{mmol}$ scale and purification by flash column chromatography on silica gel $(\mathrm{PE} / \mathrm{EtOAc}=5 / 1)$ afforded 30 as a yellow solid $(94.4 \mathrm{mg}, 99 \%)$. $\mathbf{R}_{\mathbf{f}}=0.14(\mathrm{PE} / \mathrm{EtOAc}=5 / 1) ;{ }^{1} \mathbf{H} \mathbf{N M R}\left(400 \mathrm{MHz}, \mathrm{CDCl}_{3}\right): \delta 5.40$ (bs, $1 \mathrm{H}), 3.47(\mathrm{dt}, J=12.0,4.4 \mathrm{~Hz}, 1 \mathrm{H}), 3.31(\mathrm{~s}, 3 \mathrm{H}), 3.25(\mathrm{~s}, 3 \mathrm{H})$, 3.13-3.05 (m, 1H), 2.07-1.96 (m, 2H), 1.95-1.85 (m, 2H), 1.82$1.61(\mathrm{~m}, 6 \mathrm{H}), 1.57-1.49(\mathrm{~m}, 1 \mathrm{H}), 1.46-1.40(\mathrm{~m}, 1 \mathrm{H}), 1.36-1.32(\mathrm{~m}, 5 \mathrm{H}), 1.30(\mathrm{~s}, 9 \mathrm{H}), 1.25-1.12(\mathrm{~m}$, 4H), $1.09-0.97(\mathrm{~m}, 7 \mathrm{H}), 0.87(\mathrm{~d}, J=7.6 \mathrm{~Hz}, 3 \mathrm{H}), 0.86(\mathrm{~s}, 3 \mathrm{H}), 0.58(\mathrm{~s}, 3 \mathrm{H}) ;{ }^{13} \mathbf{C} \mathbf{N M R}(100 \mathrm{MHz}$, $\left.\mathrm{CDCl}_{3}\right): \delta 172.7,80.2,77.2,56.2,56.1,55.7,55.6,51.1,44.9,42.8,40.1,40.0,38.1,36.0,35.7,35.7$, 35.4, 34.8, 31.8, 28.9, 28.3, 27.0, 25.6, 24.3, 23.7, 22.5, 20.8, 18.6, 12.1; HRMS (EI): Calcd for $\mathrm{C}_{31} \mathrm{H}_{55} \mathrm{NO}_{3}$ : 489.4182; found: 489.4181.

$N$-(tert-butyl)-4-(((R)-2,5,7,8-tetramethyl-2-((4R,8R)-4,8,12-trimethyltridecyl)chroman-6yl)oxy)butanamide (3p)

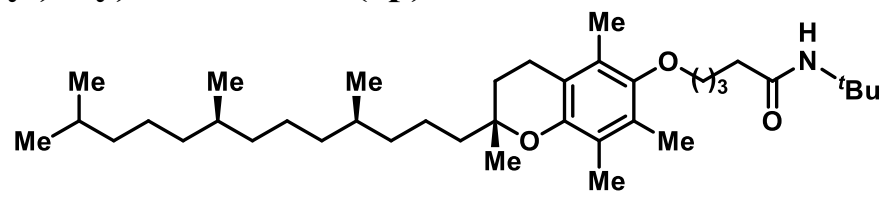

General procedure A was followed on 0.2 mmol scale and purification by flash column chromatography on silica gel $(\mathrm{PE} / \mathrm{EtOAc}=5 / 1)$ afforded $\mathbf{3 p}$ as a yellow oil (113.5 $\mathrm{mg}, 99 \%)$. $\mathbf{R}_{\mathbf{f}}$ $=0.36(\mathrm{PE} / \mathrm{EtOAc}=5 / 1) ;{ }^{1} \mathbf{H}$ NMR $\left(400 \mathrm{MHz}, \mathrm{CDCl}_{3}\right): \delta 5.49(\mathrm{bs}, 1 \mathrm{H}), 3.65(\mathrm{t}, J=6.0 \mathrm{~Hz}, 2 \mathrm{H}), 2.57$ $(\mathrm{t}, J=6.4 \mathrm{~Hz}, 2 \mathrm{H}), 2.22(\mathrm{t}, J=6.8 \mathrm{~Hz}, 2 \mathrm{H}), 2.16(\mathrm{~s}, 3 \mathrm{H}), 2.12(\mathrm{~s}, 3 \mathrm{H}), 2.08(\mathrm{~s}, 3 \mathrm{H}), 1.85-1.71(\mathrm{~m}, 6 \mathrm{H})$, $1.58-1.50(\mathrm{~m}, 3 \mathrm{H}), 1.48-1.39(\mathrm{~m}, 5 \mathrm{H}), 1.36(\mathrm{~s}, 9 \mathrm{H}), 1.29-1.20(\mathrm{~m}, 12 \mathrm{H}), 1.17-1.02(\mathrm{~m}, 7 \mathrm{H}), 0.88-$ $0.84(\mathrm{~m}, 12 \mathrm{H}) ;{ }^{13} \mathbf{C}$ NMR $\left(100 \mathrm{MHz}, \mathrm{CDCl}_{3}\right): \delta 172.4,148.3,147.8,127.8,125.8,122.9,117.6,74.9$, 72. 9, 51.2, 40.2, 39.5, 37.7, 37.6, 37.5, 37.4, 32.9, 32.8, 31.4, 29.7, 29.0, 28.1, 24.9, 24.5, 24.0, 22.9, 22.8, 22.7, 21.1, 20.8, 19.9, 19.8, 12.9, 12.0, 11.9; HRMS (EI): Calcd for $\mathrm{C}_{38} \mathrm{H}_{67} \mathrm{NO}_{3}$ : 585.5121; found: 585.5120 .

tert-butyl 4-(tert-butylcarbamoyl)piperidine-1-carboxylate (3q) 


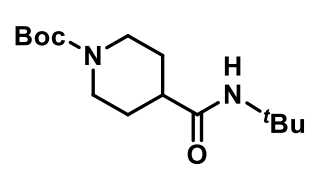

General procedure B was followed on $0.3 \mathrm{mmol}$ scale and purification by flash column chromatography on silica gel $(\mathrm{PE} / \mathrm{EtOAc}=2 / 1)$ afforded $\mathbf{3 q}$ as a yellow solid $(78.1 \mathrm{mg}, 92 \%) . \mathbf{R}_{\mathbf{f}}=0.27(\mathrm{PE} / \mathrm{EtOAc}=2 / 1) ;{ }^{1} \mathbf{H} \mathbf{~ N M R}\left(400 \mathrm{MHz}, \mathrm{CDCl}_{3}\right)$ : $\delta 5.29(\mathrm{bs}, 1 \mathrm{H}), 4.13-4.09(\mathrm{~m}, 2 \mathrm{H}), 2.70(\mathrm{t}, J=12.4 \mathrm{~Hz}, 2 \mathrm{H}), 2.09(\mathrm{t}, J=11.6 \mathrm{~Hz}$, $1 \mathrm{H}), 1.75(\mathrm{~d}, J=12.4 \mathrm{~Hz}, 2 \mathrm{H}), 1.63-1.53(\mathrm{~m}, 2 \mathrm{H}), 1.43$ (s, 9H), 1.32 (s, 9H); ${ }^{13} \mathrm{C}$ NMR $(100 \mathrm{MHz}$, $\left.\mathrm{CDCl}_{3}\right): \delta 173.9,154.8,79.7,51.2,44.2,43.4,28.9,28.8,28.5$. The spectral data are consistent with those reported in the literature.

\section{$N$-(tert-butyl)-1-tosylpiperidine-4-carboxamide (3r)}

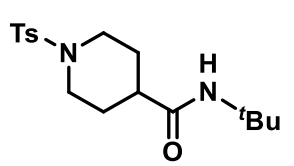

General procedure B was followed on $0.3 \mathrm{mmol}$ scale and purification by flash column chromatography on silica gel $(\mathrm{PE} / \mathrm{EtOAc}=3 / 1)$ afforded $\mathbf{3 r}$ as a white solid $(96.3 \mathrm{mg}, 95 \%)$. $\mathbf{R}_{\mathbf{f}}=0.24(\mathrm{PE} / \mathrm{EtOAc}=3 / 1) ;{ }^{1} \mathbf{H} \mathbf{~ N M R}\left(400 \mathrm{MHz}, \mathrm{CDCl}_{3}\right)$ : $\delta 7.60(\mathrm{~d}, J=8.0 \mathrm{~Hz}, 2 \mathrm{H}), 7.30(\mathrm{~d}, J=8.4 \mathrm{~Hz}, 2 \mathrm{H}), 5.29(\mathrm{bs}, 1 \mathrm{H}), 3.72(\mathrm{dt}, J=$ $11.6,3.2 \mathrm{~Hz}, 2 \mathrm{H}), 2.41(\mathrm{~s}, 3 \mathrm{H}), 2.31(\mathrm{td}, J=11.2,2.8 \mathrm{~Hz}, 2 \mathrm{H}), 1.93-1.86(\mathrm{~m}, 1 \mathrm{H}), 1.84-1.69(\mathrm{~m}$, 4H), 1.28 (s, 9H); $\left.{ }^{13} \mathbf{C ~ N M R ~ ( 1 0 0 ~ M H z , ~} \mathrm{CDCl}_{3}\right): \delta 173.2,143.7,133.2,129.7,127.7,51.2,45.6$, 43.0, 28.8, 28.3, 21.6. The spectral data are consistent with those reported in the literature.

\section{benzyl 4-(tert-butylcarbamoyl)piperidine-1-carboxylate (3s)}

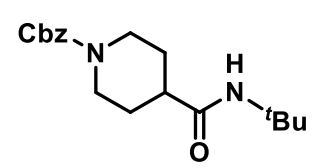

General procedure B was followed on $0.3 \mathrm{mmol}$ scale and purification by flash column chromatography on silica gel $(\mathrm{PE} / \mathrm{EtOAc}=5 / 1)$ afforded 3s as a light yellow solid $(72.7 \mathrm{mg}, 76 \%)$. Rf $=0.07(\mathrm{PE} / \mathrm{EtOAc}=5 / 1) ;{ }^{1} \mathbf{H}$ NMR $(400 \mathrm{MHz}$, $\left.\mathrm{CDCl}_{3}\right): \delta 7.30-7.20(\mathrm{~m}, 5 \mathrm{H}), 5.36(\mathrm{br} \mathrm{s}, 1 \mathrm{H}), 5.04(\mathrm{~s}, 2 \mathrm{H}), 4.12(\mathrm{bs}, 2 \mathrm{H}), 2.72(\mathrm{bs}$, $2 \mathrm{H}), 2.06(\mathrm{tt}, J=11.2,3.6 \mathrm{~Hz}, 1 \mathrm{H}), 1.70(\mathrm{~d}, J=11.2 \mathrm{~Hz}, 2 \mathrm{H}), 1.55(\mathrm{qd}, J=12.0,3.6 \mathrm{~Hz}, 2 \mathrm{H}), 1.25(\mathrm{~s}$, 9H); ${ }^{13}$ C NMR (100 MHz, $\left.\mathrm{CDCl}_{3}\right): \delta 173.6,155.2,136.8,128.5,128.0,127.9,67.1,51.1,43.8,43.5$, 28.8, 28.7. The spectral data are consistent with those reported in the literature. ${ }^{[25]}$

\section{$\mathbf{N}$-(tert-butyl)cyclopentanecarboxamide (3t)}

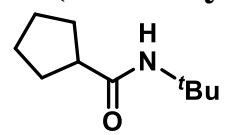

General procedure B was followed on $0.3 \mathrm{mmol}$ scale and purification by flash

column chromatography on silica gel $(\mathrm{PE} / \mathrm{EtOAc}=5 / 1)$ afforded $\mathbf{3 t}$ as a white solid $(39.3 \mathrm{mg}, 78 \%) . \mathbf{R}_{\mathbf{f}}=0.50(\mathrm{PE} / \mathrm{EtOAc}=5 / 1) ;{ }^{1} \mathbf{H} \mathbf{~ N M R}\left(400 \mathrm{MHz}, \mathrm{CDCl}_{3}\right): \delta 5.32(\mathrm{br}$ s, $1 \mathrm{H}$ ), 2.40 (quin, $J=8.0 \mathrm{~Hz}, 1 \mathrm{H}), 1.82-1.70(\mathrm{~m}, 6 \mathrm{H}), 1.57-1.47(\mathrm{~m}, 2 \mathrm{H}), 1.32(\mathrm{~s}, 9 \mathrm{H}) ;{ }^{13} \mathbf{C}$ NMR $\left(100 \mathrm{MHz}, \mathrm{CDCl}_{3}\right): \delta 175.8,51.0,46.6,30.5,29.0,26.1$. The spectral data are consistent with those reported in the literature. ${ }^{[26]}$

\section{$\mathrm{N}$-(tert-butyl)tetrahydro-2H-pyran-4-carboxamide (3u)}

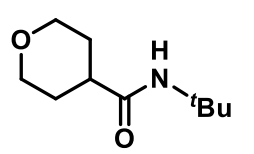

General procedure B was followed on $0.3 \mathrm{mmol}$ scale and purification by flash column chromatography on silica gel $(\mathrm{PE} / \mathrm{EtOAc}=1 / 1)$ afforded $\mathbf{3 u}$ as a white solid $(56.2 \mathrm{mg}, 98 \%) . \mathbf{R}_{\mathbf{f}}=0.37(\mathrm{PE} / \mathrm{EtOAc}=1 / 1) ;{ }^{1} \mathbf{H} \mathbf{N M R}\left(400 \mathrm{MHz}, \mathrm{CDCl}_{3}\right): \delta 5.29$ (bs, $1 \mathrm{H}), 4.00$ (d, $J=11.2 \mathrm{~Hz}, 2 \mathrm{H}), 3.38(\mathrm{dt}, J=11.2,2.4 \mathrm{~Hz}, 2 \mathrm{H}), 2.25-2.18(\mathrm{~m}, 1 \mathrm{H})$, $1.81-1.68(\mathrm{~m}, 4 \mathrm{H}), 1.33$ (s, 9H); ${ }^{13} \mathbf{C}$ NMR (100 MHz, $\left.\mathrm{CDCl}_{3}\right): \delta 173.8,67.4,51.2,43.1,29.5,29.0$. The spectral data are consistent with those reported in the literature. ${ }^{[22]}$ 


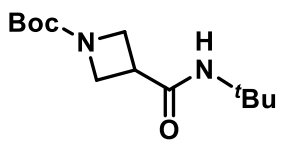

General procedure B was followed on $0.3 \mathrm{mmol}$ scale and purification by flash column chromatography on silica gel $(\mathrm{PE} / \mathrm{EtOAc}=3 / 1)$ afforded $\mathbf{3 v}$ as a yellow solid $(75.8 \mathrm{mg}, 99 \%) . \mathbf{R}_{\mathbf{f}}=0.26(\mathrm{PE} / \mathrm{EtOAc}=3 / 1) ;{ }^{1} \mathbf{H} \mathbf{~ N M R}\left(400 \mathrm{MHz}, \mathrm{CDCl}_{3}\right): \delta$ 5.75 (bs, $1 \mathrm{H}), 4.00(\mathrm{dd}, J=7.6,6.4 \mathrm{~Hz}, 2 \mathrm{H}), 3.92(\mathrm{t}, J=8.4 \mathrm{~Hz}, 2 \mathrm{H}), 3.11-3.04(\mathrm{~m}, 1 \mathrm{H}), 1.37$ (s, 9H), 1.29 (s, 9H); ${ }^{13} \mathbf{C}$ NMR (100 MHz, $\left.\mathrm{CDCl}_{3}\right): \delta 171.0,156.3,79.6,51.7,51.4,33.8,28.8,28.4$; HRMS (EI): Calcd for $\mathrm{C}_{13} \mathrm{H}_{24} \mathrm{~N}_{2} \mathrm{O}_{3}: 256.1787$; found: 256.1786 .

\section{$N$-(tert-butyl)-2-methylbutanamide (3w)}

General procedure B was followed on $0.3 \mathrm{mmol}$ scale and purification by flash $\underbrace{M e}_{0} N_{t_{B u}}^{H}$ column chromatography on silica gel $(\mathrm{PE} / \mathrm{EtOAc}=5 / 1)$ afforded $\mathbf{3} \mathbf{w}$ as a light yellow solid (36.8 mg, 78\%). $\mathbf{R}_{\mathbf{f}}=0.40(\mathrm{PE} / \mathrm{EtOAc}=5 / 1) ;{ }^{1} \mathbf{H} \mathbf{~ N M R}\left(400 \mathrm{MHz}, \mathrm{CDCl}_{3}\right): \delta$ 5.29 (bs, 1H), 1.96 (sext, $J=6.8 \mathrm{~Hz}, 1 \mathrm{H}), 1.66-1.55(\mathrm{~m}, 1 \mathrm{H}), 1.44-1.37(\mathrm{~m}, 1 \mathrm{H})$, $1.34(\mathrm{~s}, 9 \mathrm{H}), 1.08(\mathrm{~d}, J=6.8 \mathrm{~Hz}, 3 \mathrm{H}), 0.88(\mathrm{t}, J=7.2 \mathrm{~Hz}, 3 \mathrm{H}) ;{ }^{13} \mathbf{C} \mathbf{N M R}\left(100 \mathrm{MHz}, \mathrm{CDCl}_{3}\right): \delta 176.1$, $51.1,44.1,29.0,27.6,17.8,12.1$. The spectral data are consistent with those reported in the literature. ${ }^{[27]}$

\section{tert-butyl 4-((2-methyl-4-phenylbutan-2-yl)carbamoyl)piperidine-1-carboxylate (3x)}

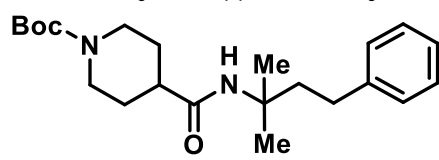

General procedure B was followed on $0.3 \mathrm{mmol}$ scale and purification by flash column chromatography on silica gel $(\mathrm{PE} / \mathrm{EtOAc}=3 / 1)$ afforded $\mathbf{3 x}$ as a yellow solid (103.3 mg, 92\%). $\mathbf{R}_{\mathbf{f}}=0.37(\mathrm{PE} /$ EtOAc $=3 / 1) ;{ }^{1} \mathbf{H}$ NMR $\left(400 \mathrm{MHz}, \mathrm{CDCl}_{3}\right): \delta 7.20-7.16(\mathrm{~m}, 2 \mathrm{H}), 7.10-7.06(\mathrm{~m}, 3 \mathrm{H}), 5.33(\mathrm{bs}$, $1 \mathrm{H}), 4.06-4.02(\mathrm{~m}, 2 \mathrm{H}), 2.61(\mathrm{t}, J=12.4 \mathrm{~Hz}, 2 \mathrm{H}), 2.49-2.45(\mathrm{~m}, 2 \mathrm{H}), 2.05-1.94(\mathrm{~m}, 3 \mathrm{H}), 1.64(\mathrm{~d}, J=$ $11.2 \mathrm{~Hz}, 2 \mathrm{H}), 1.50$ (ddt, $J=12.8,12.0,4.4 \mathrm{~Hz}, 2 \mathrm{H}), 1.37$ (s, 9H), $1.28(\mathrm{~s}, 6 \mathrm{H}) ;{ }^{13} \mathrm{C} \mathrm{NMR}(100 \mathrm{MHz}$, $\left.\mathrm{CDCl}_{3}\right): \delta 173.8,154.7,142.2,128.4,128.4,125.8,79.6,53.6,44.0,43.2,41.6,30.8,28.8,28.4,27.2$; HRMS (EI): Calcd for $\mathrm{C}_{22} \mathrm{H}_{34} \mathrm{~N}_{2} \mathrm{O}_{3}: 374.2569$; found: 374.2568 .

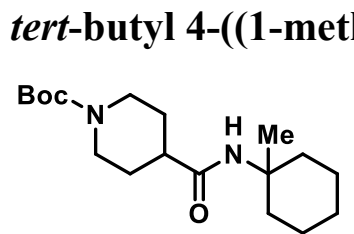

\section{(3y)}

General procedure B was followed on $0.24 \mathrm{mmol}$ scale and purification by flash column chromatography on silica gel $(\mathrm{PE} / \mathrm{EtOAc}=5 / 1)$ afforded $\mathbf{3 y}$ as a white solid (51.5 mg, 66\%). Rf $=0.23(\mathrm{PE} / \mathrm{EtOAc}=5 / 1) ;{ }^{1} \mathbf{H}$ NMR $\left(400 \mathrm{MHz}, \mathrm{CDCl}_{3}\right)$ : $\delta 5.22(\mathrm{bs}, 1 \mathrm{H}), 4.09(\mathrm{~d}, J=12.8 \mathrm{~Hz}, 2 \mathrm{H}), 2.69(\mathrm{t}, J=12.0 \mathrm{~Hz}, 2 \mathrm{H}), 2.14(\mathrm{tt}, J=$ 11.6, 3.2 Hz, 1H), 2.01-1.96 (m, 2H), $1.75(\mathrm{~d}, J=12.4 \mathrm{~Hz}, 2 \mathrm{H}), 1.57$ (ddd, $J=24.4,12.4,4.0 \mathrm{~Hz}, 2 \mathrm{H})$ $1.48(\mathrm{t}, J=6.4 \mathrm{~Hz}, 2 \mathrm{H}), 1.41(\mathrm{~s}, 9 \mathrm{H}), 1.37-1.31(\mathrm{~m}, 4 \mathrm{H}), 1.30(\mathrm{~s}, 3 \mathrm{H}), 1.25-1.22(\mathrm{~m}, 2 \mathrm{H}) ;{ }^{13} \mathbf{C} \mathbf{N M R}$ $\left(100 \mathrm{MHz} \mathrm{CDCl}_{3}\right): \delta 173.8,154.8,79.6,53.2,44.2,43.4,36.7,28.9,28.5,26.6,25.6,22.1$; HRMS (EI): Calcd for $\mathrm{C}_{18} \mathrm{H}_{32} \mathrm{~N}_{2} \mathrm{O}_{3}: 324.2413$; found: 324.2412 .

\section{tert-butyl 4-(((3s,5s,7s)-adamantan-1-yl)carbamoyl)piperidine-1-carboxylate (3z)}

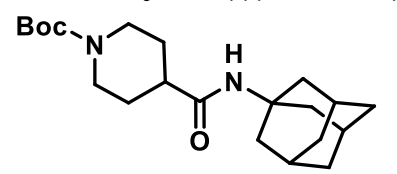

General procedure $\mathrm{B}$ was followed on $0.3 \mathrm{mmol}$ scale and purification by flash column chromatography on silica gel $(\mathrm{PE} / \mathrm{EtOAc}=2 / 1)$ afforded $\mathbf{3 z}$ as a yellow solid $(81.5 \mathrm{mg}, 75 \%) . \mathbf{R} \mathbf{f}=0.62(\mathrm{PE} /$ EtOAc $=2 / 1) ;{ }^{1} \mathbf{H}$ NMR $(400$ $\left.\mathrm{MHz}, \mathrm{CDCl}_{3}\right): \delta 5.23(\mathrm{bs}, 1 \mathrm{H}), 4.08-4.05(\mathrm{~m}, 2 \mathrm{H}), 2.67(\mathrm{t}, J=12.0 \mathrm{~Hz}, 2 \mathrm{H})$, 2.11-2.05 (m, 1H), 2.04-1.99 (m, 3H), $1.93(\mathrm{~d}, J=2.8 \mathrm{~Hz}, 6 \mathrm{H}), 1.71(\mathrm{dd}, J=12.8,2.0 \mathrm{~Hz}, 2 \mathrm{H}), 1.62$ $(\mathrm{t}, J=2.8 \mathrm{~Hz}, 6 \mathrm{H}) 1.59-1.49(\mathrm{~m}, 2 \mathrm{H}), 1.40(\mathrm{~s}, 9 \mathrm{H}) ;{ }^{13} \mathbf{C} \mathbf{N M R}\left(100 \mathrm{MHz}, \mathrm{CDCl}_{3}\right): \delta 173.7,154.7,79.5$, 
$51.8,44.0,43.3,41.7,36.4,29.4,28.8,28.5$. The spectral data are consistent with those reported in the literature.

tert-butyl 4-((8-(benzoyloxy)-2,6-dimethyloctan-2-yl)carbamoyl)piperidine-1-carboxylate (3aa)

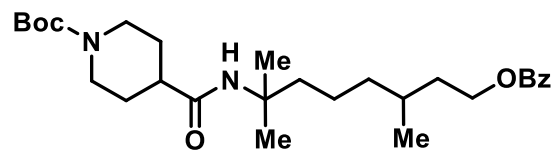

General procedure $\mathrm{B}$ was followed on $0.3 \mathrm{mmol}$ scale and purification by flash column chromatography on silica gel $(\mathrm{PE} / \mathrm{EtOAc}=3 / 1)$ afforded $3 \mathbf{a a}$ as a yellow oil $(77.5 \mathrm{mg}, 53 \%)$. $\mathbf{R}_{\mathbf{f}}$ $=0.72(\mathrm{PE} / \mathrm{EtOAc}=1 / 1) ;{ }^{1} \mathbf{H}$ NMR $\left(400 \mathrm{MHz}, \mathrm{CDCl}_{3}\right): \delta 8.01-$ $7.99(\mathrm{~m}, 2 \mathrm{H}), 7.52(\mathrm{tt}, J=7.2,1.2 \mathrm{~Hz}, 1 \mathrm{H}), 7.40(\mathrm{t}, J=7.6 \mathrm{~Hz}, 2 \mathrm{H}), 5.26(\mathrm{bs}, 1 \mathrm{H}), 4.37-4.27(\mathrm{~m}, 2 \mathrm{H})$, $4.11-4.05(\mathrm{~m}, 2 \mathrm{H}), 2.67(\mathrm{t}, J=12.0 \mathrm{~Hz}, 2 \mathrm{H}), 2.10(\mathrm{tt}, J=11.6,3.2 \mathrm{~Hz}, 1 \mathrm{H}), 1.80-1.51(\mathrm{~m}, 9 \mathrm{H}), 1.41$ $(\mathrm{s}, 9 \mathrm{H}), 1.25(\mathrm{~s}, 6 \mathrm{H}), 1.24-1.20(\mathrm{~m}, 4 \mathrm{H}), 0.92(\mathrm{~d}, J=6.4 \mathrm{~Hz}, 3 \mathrm{H}) ;{ }^{13} \mathbf{C}$ NMR $\left(100 \mathrm{MHz}, \mathrm{CDCl}_{3}\right): \delta$ $173.8,166.7,154.7,132.9,130.5,129.5,128.4,79.6,63.5,60.4,53.6,44.1,43.3,40.2,37.2,35.6,29.9$, 28.8, 28.5, 27.1, 21.4, 21.1, 19.6, 14.2; HRMS (EI): Calcd for $\mathrm{C}_{28} \mathrm{H}_{44} \mathrm{~N}_{2} \mathrm{O}_{5}$ : 488.3250; found: 488.3253 .

\section{Derivatization Experiments}

\section{Direct formation of primary amide from alkyl iodide 1a}
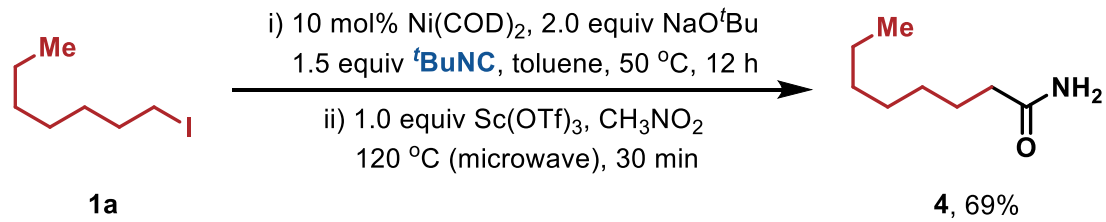

To a solution of $\mathrm{Ni}(\mathrm{COD})_{2}(10 \mathrm{~mol} \%, 0.03 \mathrm{mmol}, 8.3 \mathrm{mg})$ and sodium tert-butoxide (2.0 equiv, $0.6 \mathrm{mmol}, 57.7 \mathrm{mg})$ in toluene $(0.1 \mathrm{M}, 3.0 \mathrm{~mL})$ was added tert-butyl isocyanide (1.5 equiv, $0.45 \mathrm{mmol}$, $50.9 \mu \mathrm{L})$ and alkyl iodide $1 \mathrm{a}(1.0$ equiv, $0.3 \mathrm{mmol}, 49.2 \mu \mathrm{L})$ in turn. The reaction mixture was stirred at a $50{ }^{\circ} \mathrm{C}$ oil bath until complete by TLC analysis. When the starting material was consumed, the reaction was filtered through silica gel. The filtrate was concentrated in vacuo and the residue was diluted with $\mathrm{CH}_{3} \mathrm{NO}_{2}(0.3 \mathrm{M}, 1.0 \mathrm{~mL})$ in a microwave vial containing a stirring bar. Then $\mathrm{Sc}(\mathrm{OTf})_{3}$ (1.0 equiv. $0.3 \mathrm{mmol}, 147.6 \mathrm{mg}$ ) was added, and the resulting solution was heated at $120{ }^{\circ} \mathrm{C}$ for $30 \mathrm{~min}$ using a Biotage Microwave Reactor. The reaction mixture was cooled to room temperature and was concentrated in vacuo. The residue was dissolved in DCM, washed with water, dried over $\mathrm{Na}_{2} \mathrm{SO}_{4}$ and then filtered. The filtrate was concentrated in vacuo and the residue was purified by flash column chromatography on silica gel to afford 4 as a white solid $(29.6 \mathrm{mg}, 69 \%) . \mathbf{R}_{\mathbf{f}}=0.41(\mathrm{PE} / \mathrm{EtOAc}=1 / 1)$; ${ }^{1}$ H NMR $\left(400 \mathrm{MHz}, \mathrm{CDCl}_{3}\right): \delta 6.05$ (br s, 1H), 5.67 (br s, $\left.1 \mathrm{H}\right), 2.20(\mathrm{t}, J=7.6 \mathrm{~Hz}, 2 \mathrm{H}), 1.64-1.56$ (m, $2 \mathrm{H}), 1.33-1.23(\mathrm{~m}, 8 \mathrm{H}), 0.86(\mathrm{t}, J=6.4 \mathrm{~Hz}, 3 \mathrm{H})$. The spectral data are consistent with those reported in the literature. ${ }^{[28]}$

\section{Direct formation of alkyl carboxylic acid from alkyl iodide 1a}




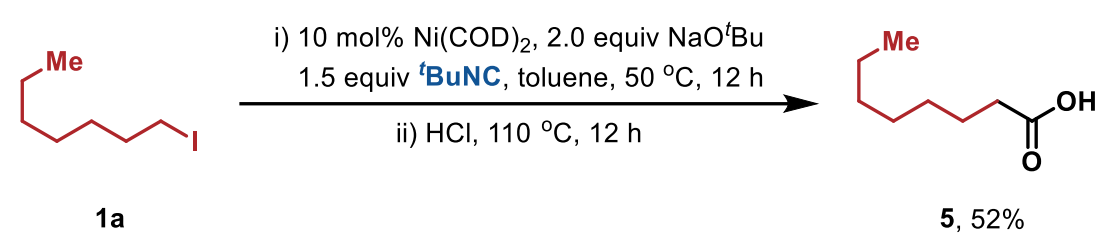

To a solution of $\mathrm{Ni}(\mathrm{COD})_{2}(10 \mathrm{~mol} \%, 0.03 \mathrm{mmol}, 8.3 \mathrm{mg})$ and sodium tert-butoxide (2.0 equiv, 0.6 mmol, $57.7 \mathrm{mg})$ in toluene $(0.1 \mathrm{M}, 3.0 \mathrm{~mL})$ was added tert-butyl isocyanide (1.5 equiv, $0.45 \mathrm{mmol}$, $50.9 \mu \mathrm{L})$ and alkyl iodide $1 \mathrm{a}(1.0$ equiv, $0.3 \mathrm{mmol}, 49.2 \mu \mathrm{L})$ in turn. The reaction mixture was stirred at a $50{ }^{\circ} \mathrm{C}$ oil bath until complete by TLC analysis. When the starting material was consumed, $12 \mathrm{M}$ aq. $\mathrm{HCl}(5.0 \mathrm{~mL})$ was added. The reaction mixture refluxed at a $110^{\circ} \mathrm{C}$ oil bath until complete by TLC analysis. Then the reaction solution was diluted with DCM, and extracted three times with DCM. The combined organic layer was dried over $\mathrm{Na}_{2} \mathrm{SO}_{4}$, and then filtered. The filtrate was concentrated in vacuo and the residue was purified by flash column chromatography on silica gel to afford $\mathbf{5}$ as a white solid (22.5 mg, 52\%). $\mathbf{R}_{\mathbf{f}}=0.40(\mathrm{PE} / \mathrm{EtOAc}=1 / 1) ;{ }^{1} \mathbf{H} \mathbf{N M R}\left(400 \mathrm{MHz}, \mathrm{CDCl}_{3}\right): \delta 2.35(\mathrm{t}, J=7.6 \mathrm{~Hz}$, $2 \mathrm{H}), 1.67-1.59(\mathrm{~m}, 2 \mathrm{H}), 1.35-1.25(\mathrm{~m}, 8 \mathrm{H}), 0.88(\mathrm{t}, J=6.8 \mathrm{~Hz}, 3 \mathrm{H}) ;{ }^{13} \mathbf{C ~ N M R}\left(100 \mathrm{MHz}, \mathrm{CDCl}_{3}\right): \delta$ $180.6,34.2,31.8,29.2,29.0,24.8,22.7,14.2$. The spectral data are consistent with those reported in the literature. ${ }^{[29]}$

\section{Preliminary Mechanistic Study}

\section{Radical rearrangement of $1 \mathrm{ab}$}

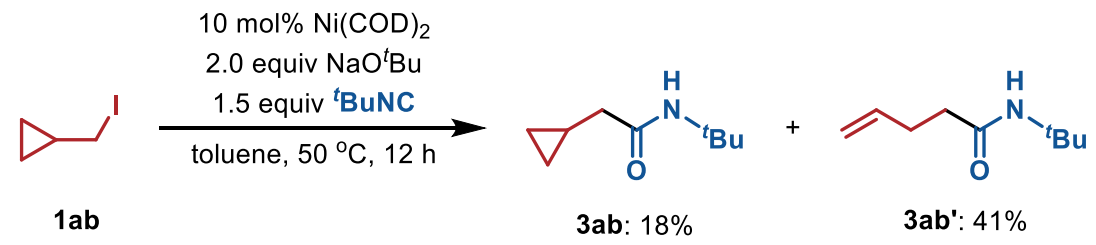

To a solution of $\mathrm{Ni}(\mathrm{COD})_{2}(10 \mathrm{~mol} \%, 0.03 \mathrm{mmol}, 8.3 \mathrm{mg})$ and sodium tert-butoxide (2.0 equiv, $0.6 \mathrm{mmol}, 57.7 \mathrm{mg})$ in toluene $(0.1 \mathrm{M}, 3.0 \mathrm{~mL})$ was added tert-butyl isocyanide (1.5 equiv, $0.45 \mathrm{mmol}$, $50.9 \mu \mathrm{L})$ and alkyl iodide $1 \mathrm{ab}(1.0$ equiv, $0.3 \mathrm{mmol}, 54.6 \mathrm{mg})$ in turn. The reaction mixture was stirred at a $50{ }^{\circ} \mathrm{C}$ oil bath until complete by TLC analysis. When the starting material was consumed, the reaction was quenched with $1 \mathrm{M}$ aq. $\mathrm{HCl}(3.0 \mathrm{~mL})$ and extracted three times with ethyl acetate. The combined organic layer was dried over $\mathrm{Na}_{2} \mathrm{SO}_{4}$, and then filtered. The filtrate was concentrated in vacuo and the residue was purified by flash column chromatography to afford 3ab with 3ab' as a yellow oil (27.5 mg, 3ab: 18\%, 3ab': 41\%). $\mathbf{R}_{\mathbf{f}}=0.43(\mathrm{PE} / \mathrm{EtOAc}=5 / 1)$.

\footnotetext{
${ }^{1} \mathrm{H}$ NMR-spectrum(400 MHz, $\left.\mathrm{CDCl}_{3}\right)$ of $3 \mathrm{ab}$ and $3 \mathrm{ab}^{\prime}$
} 


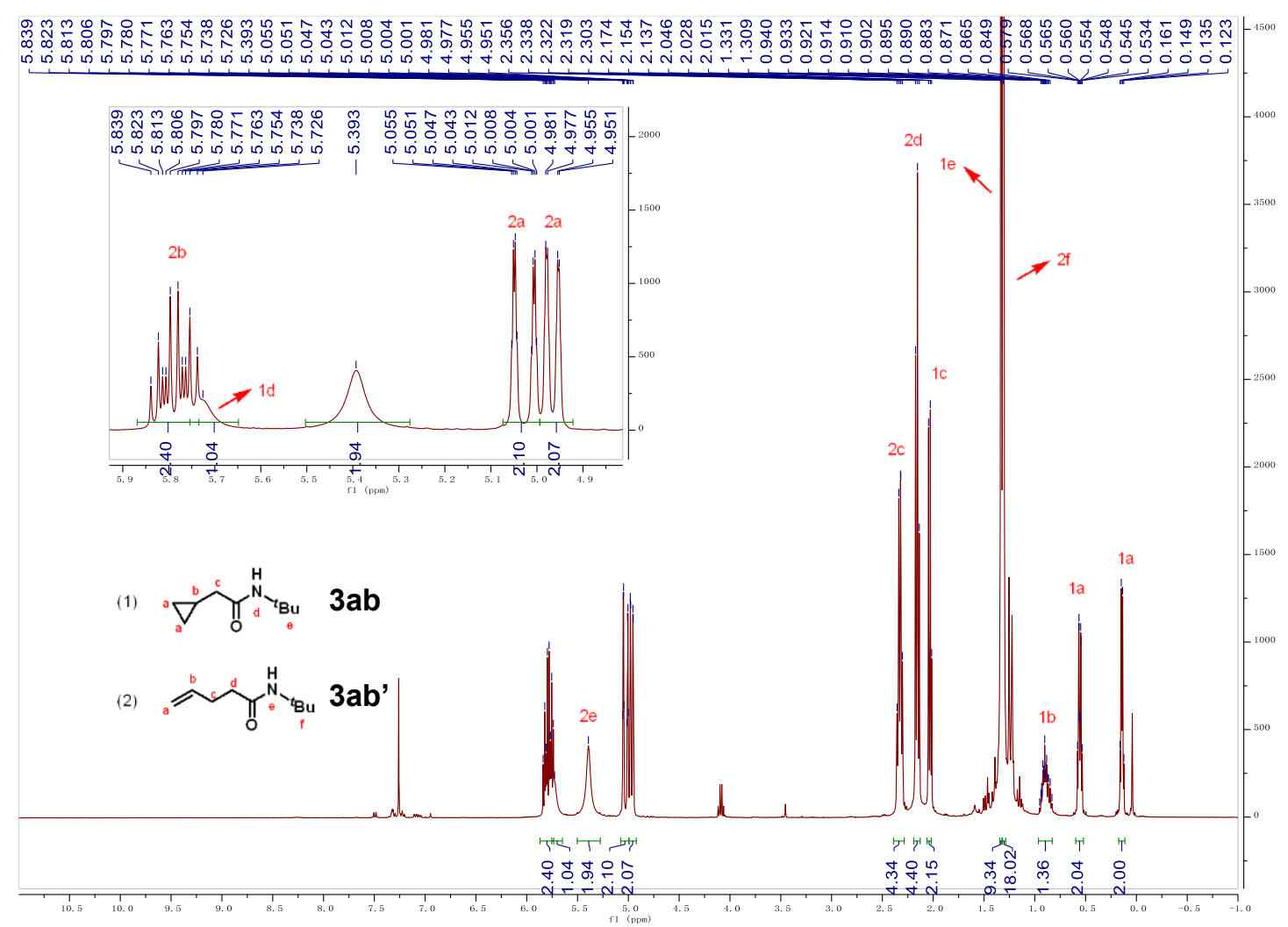


NMR Spectra

${ }^{1} \mathrm{H}$ NMR-spectrum(400 $\left.\mathrm{MHz}, \mathrm{CDCl}_{3}\right)$ of $1 \mathrm{n}$

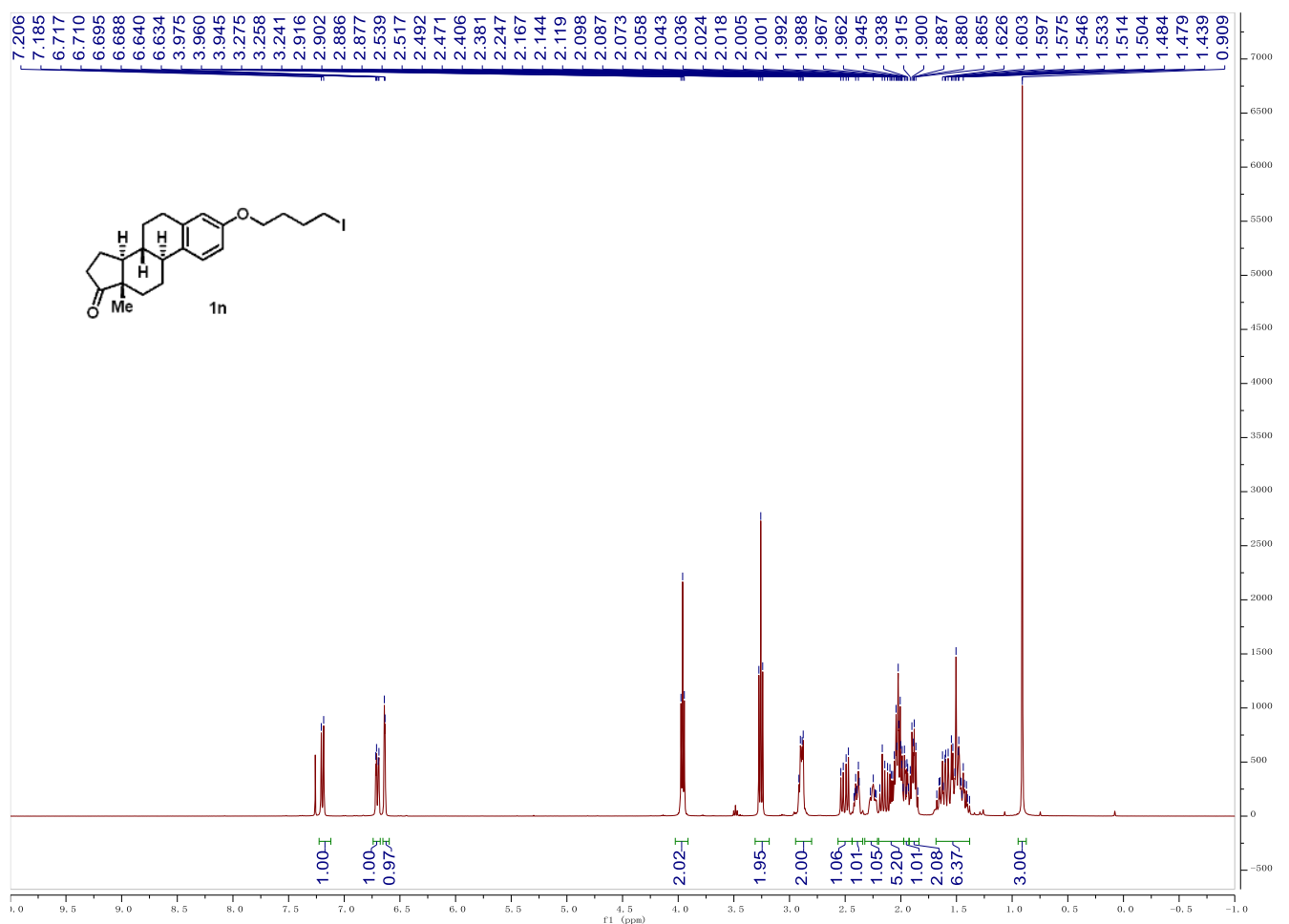

${ }^{13} \mathrm{C}$ NMR-spectrum(100 MHz, $\left.\mathrm{CDCl}_{3}\right)$ of $1 \mathrm{n}$

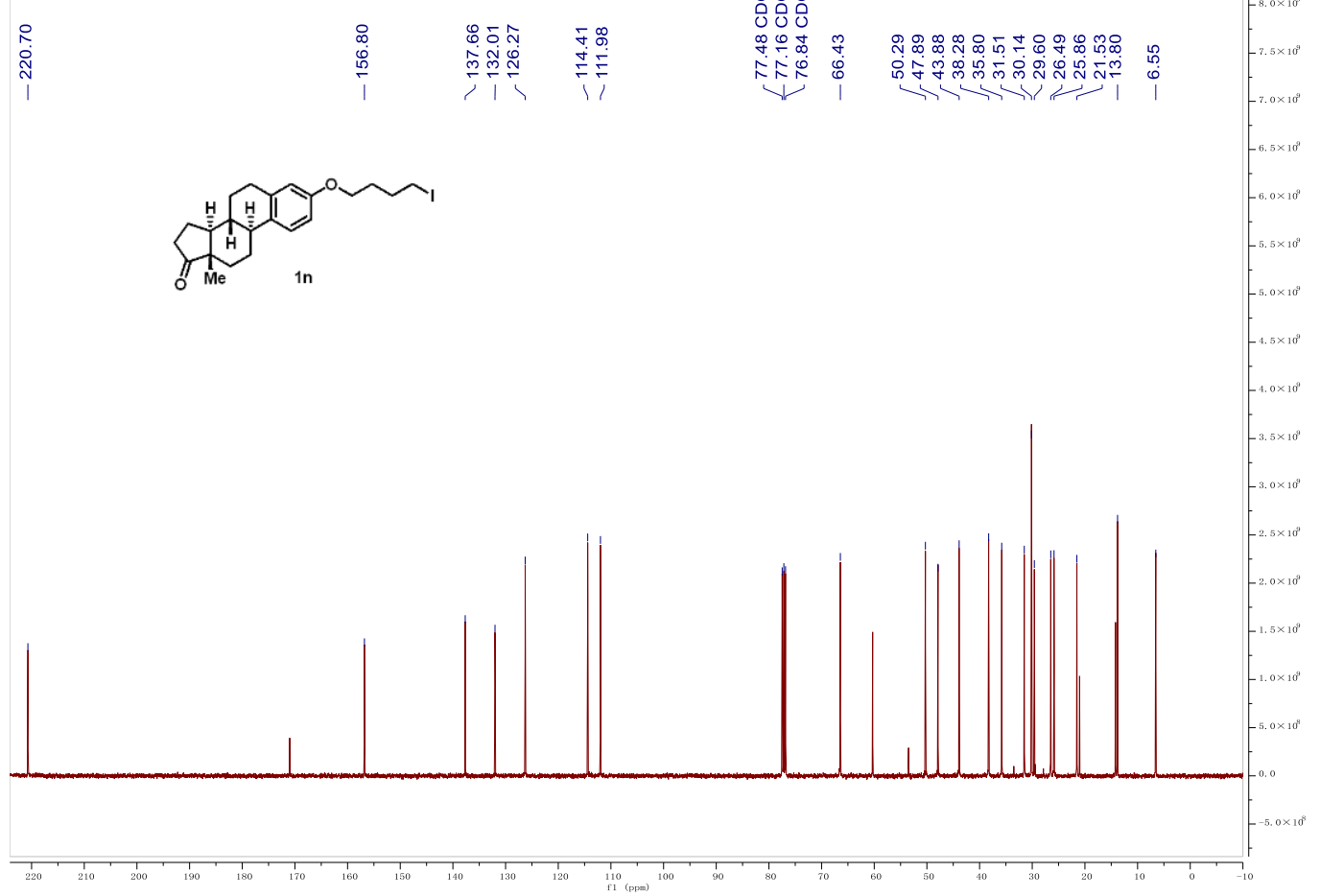


${ }^{1} \mathrm{H}$ NMR-spectrum(400 $\left.\mathrm{MHz}, \mathrm{CDCl}_{3}\right)$ of 10

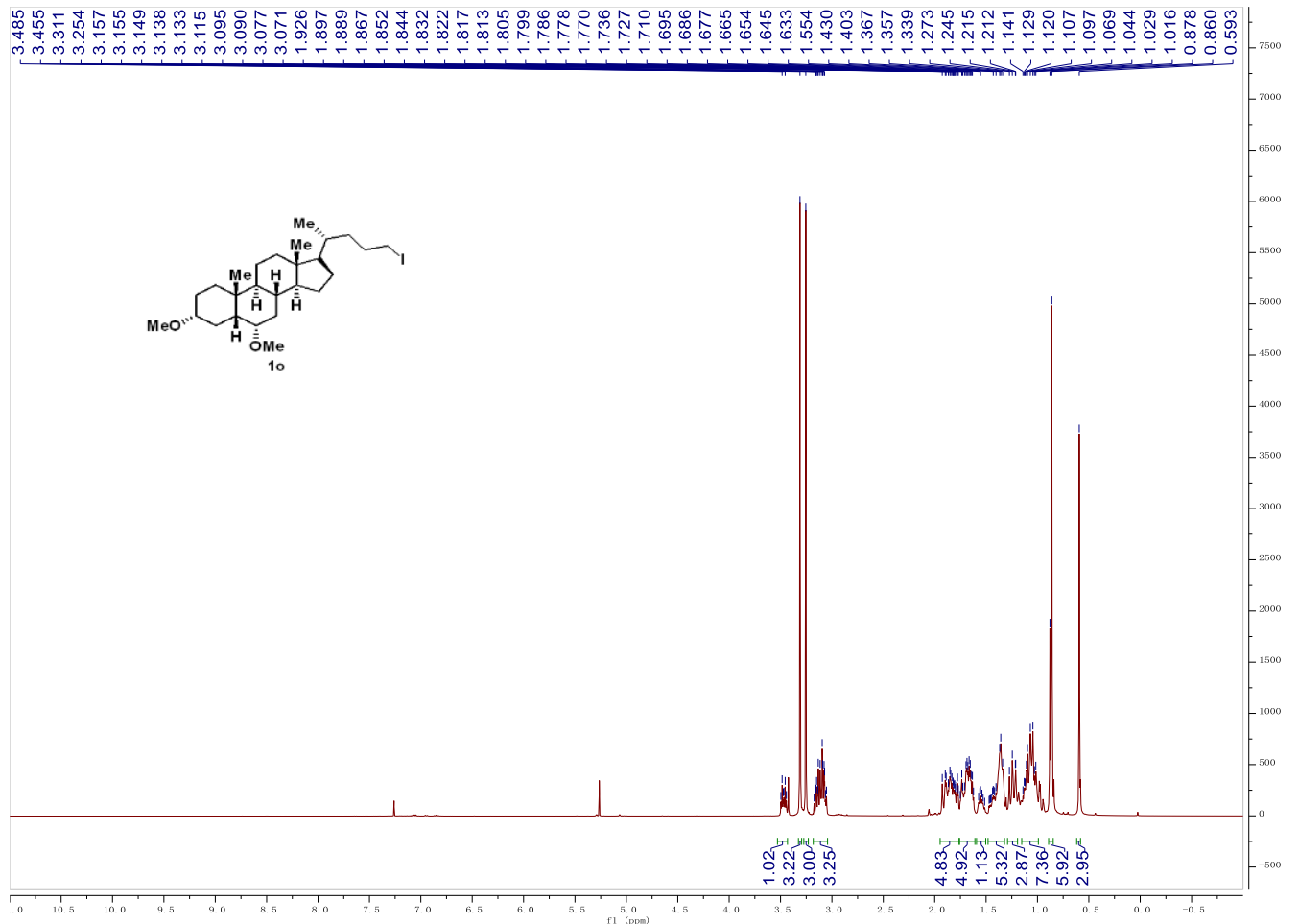

${ }^{13} \mathrm{C}$ NMR-spectrum(100 MHz, $\left.\mathrm{CDCl}_{3}\right)$ of 10

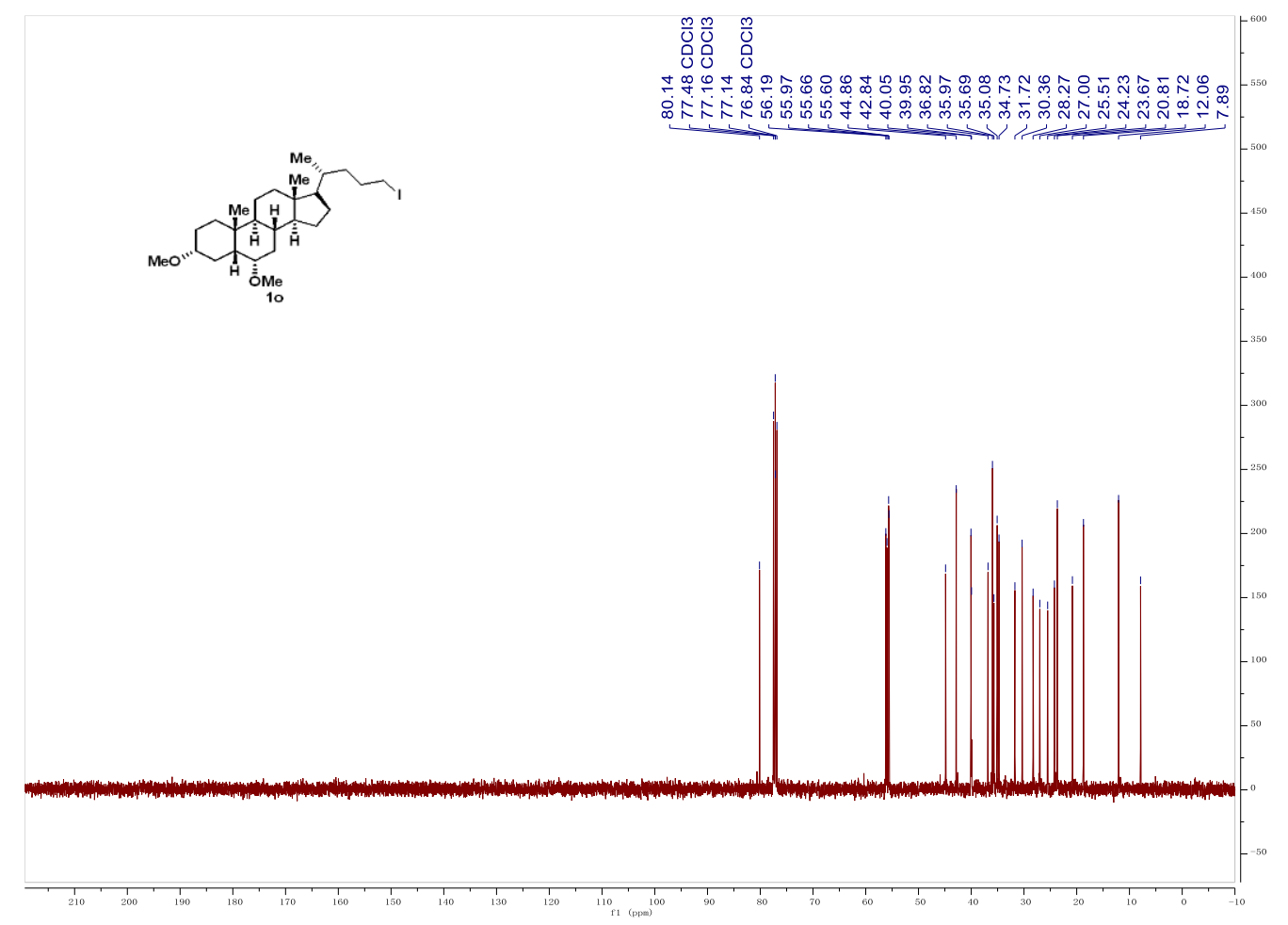


${ }^{1} \mathrm{H}$ NMR-spectrum(400 $\left.\mathrm{MHz}, \mathrm{CDCl}_{3}\right)$ of $1 \mathrm{p}$

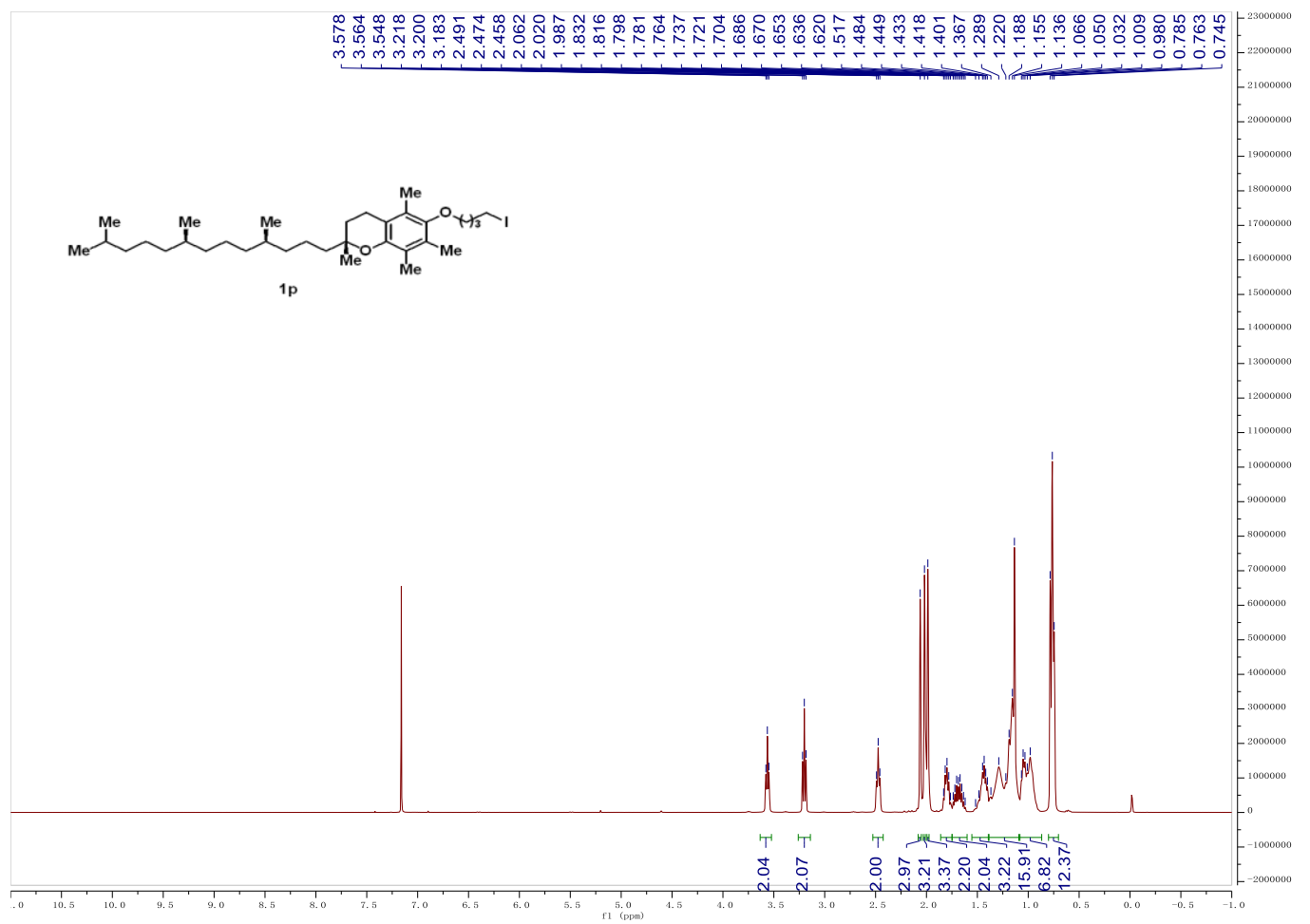

${ }^{13} \mathrm{C}$ NMR-spectrum(100 MHz, $\left.\mathrm{CDCl}_{3}\right)$ of $1 \mathrm{p}$

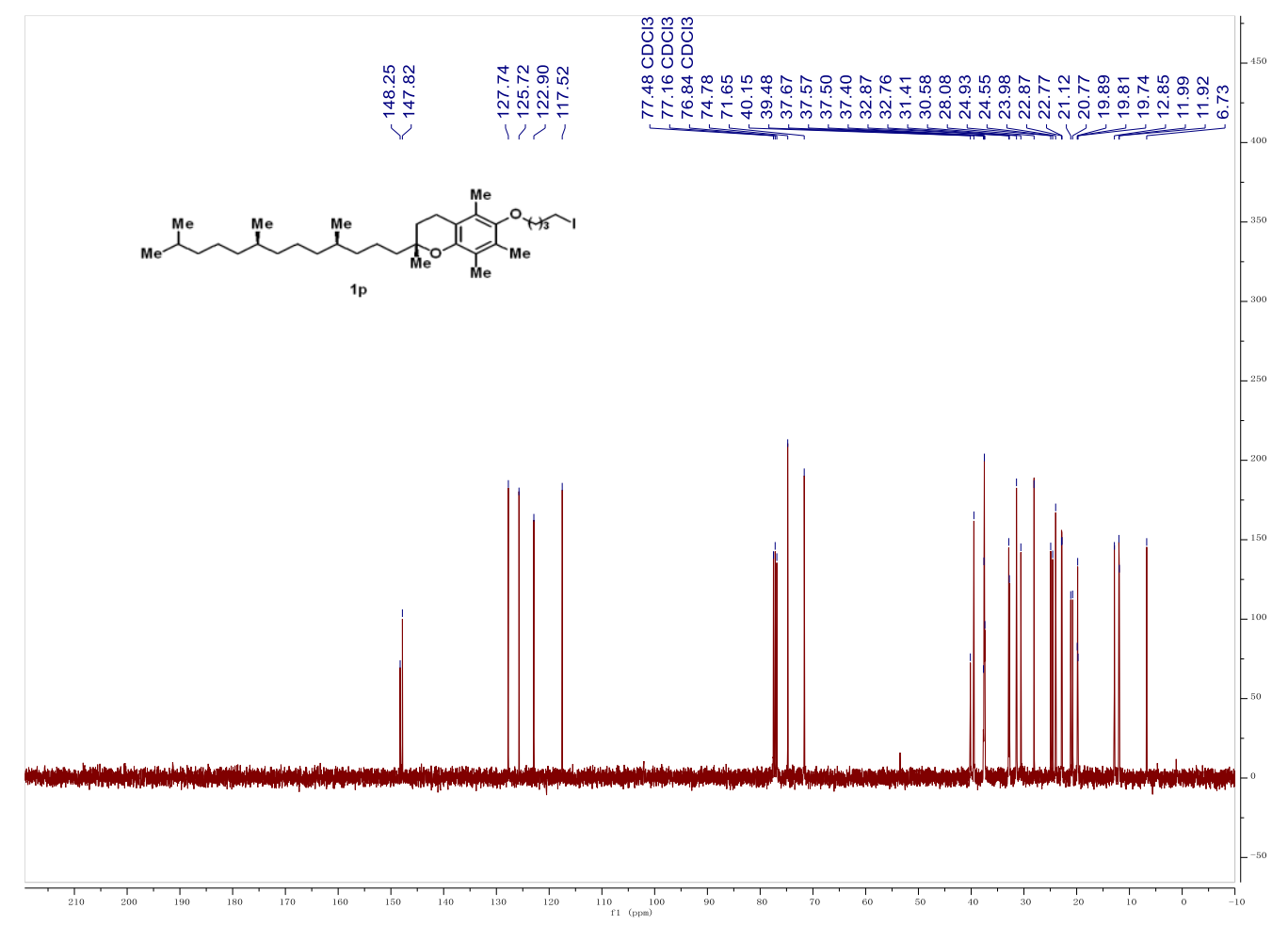


${ }^{1} \mathrm{H}$ NMR-spectrum(400 $\left.\mathrm{MHz}, \mathrm{CDCl}_{3}\right)$ of $1 \mathrm{k}$

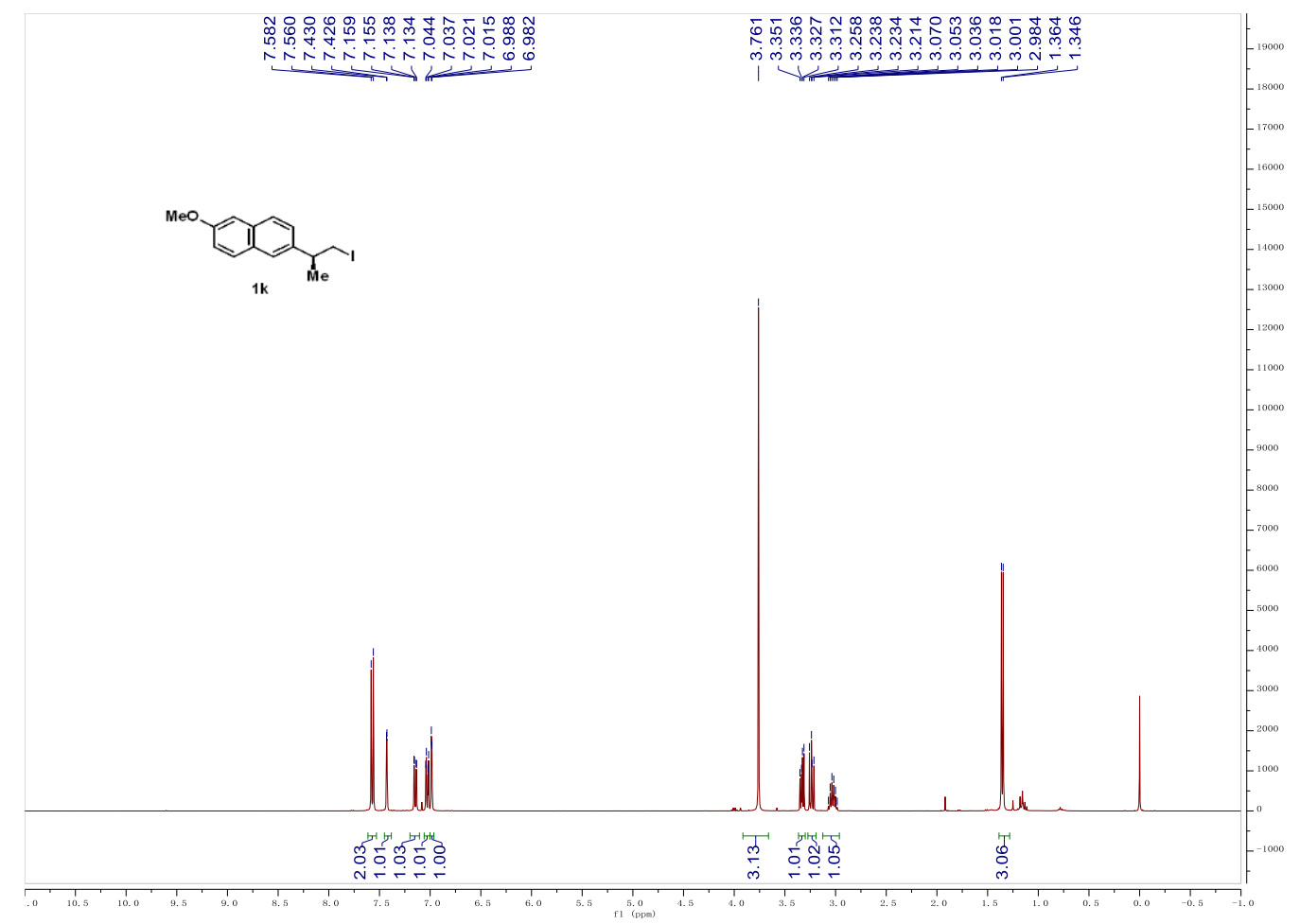

${ }^{13} \mathrm{C}$ NMR-spectrum(100 $\left.\mathrm{MHz}, \mathrm{CDCl}_{3}\right)$ of $1 \mathrm{k}$

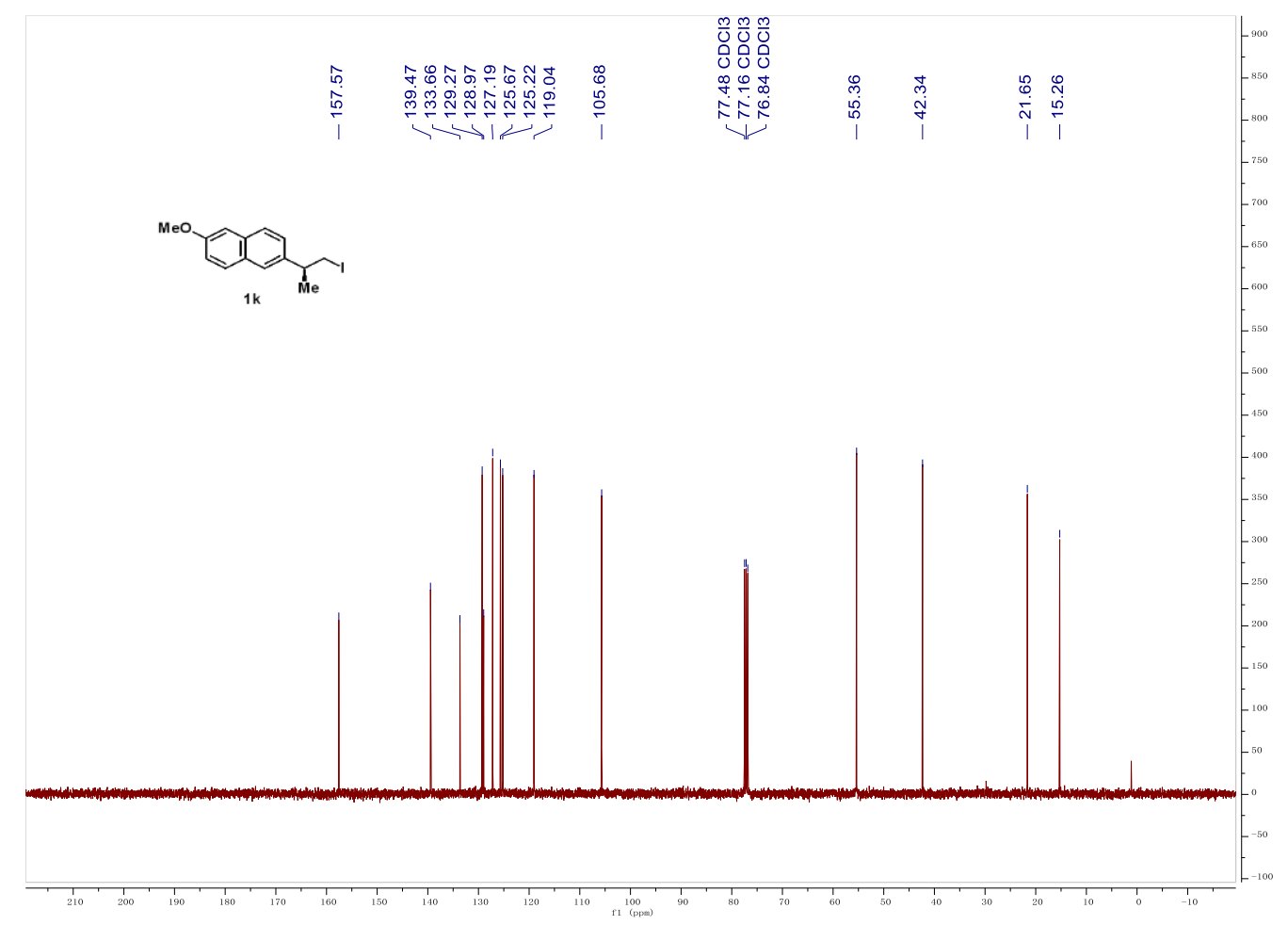


${ }^{1} \mathrm{H}$ NMR-spectrum(400 $\left.\mathrm{MHz}, \mathrm{CDCl}_{3}\right)$ of 3a

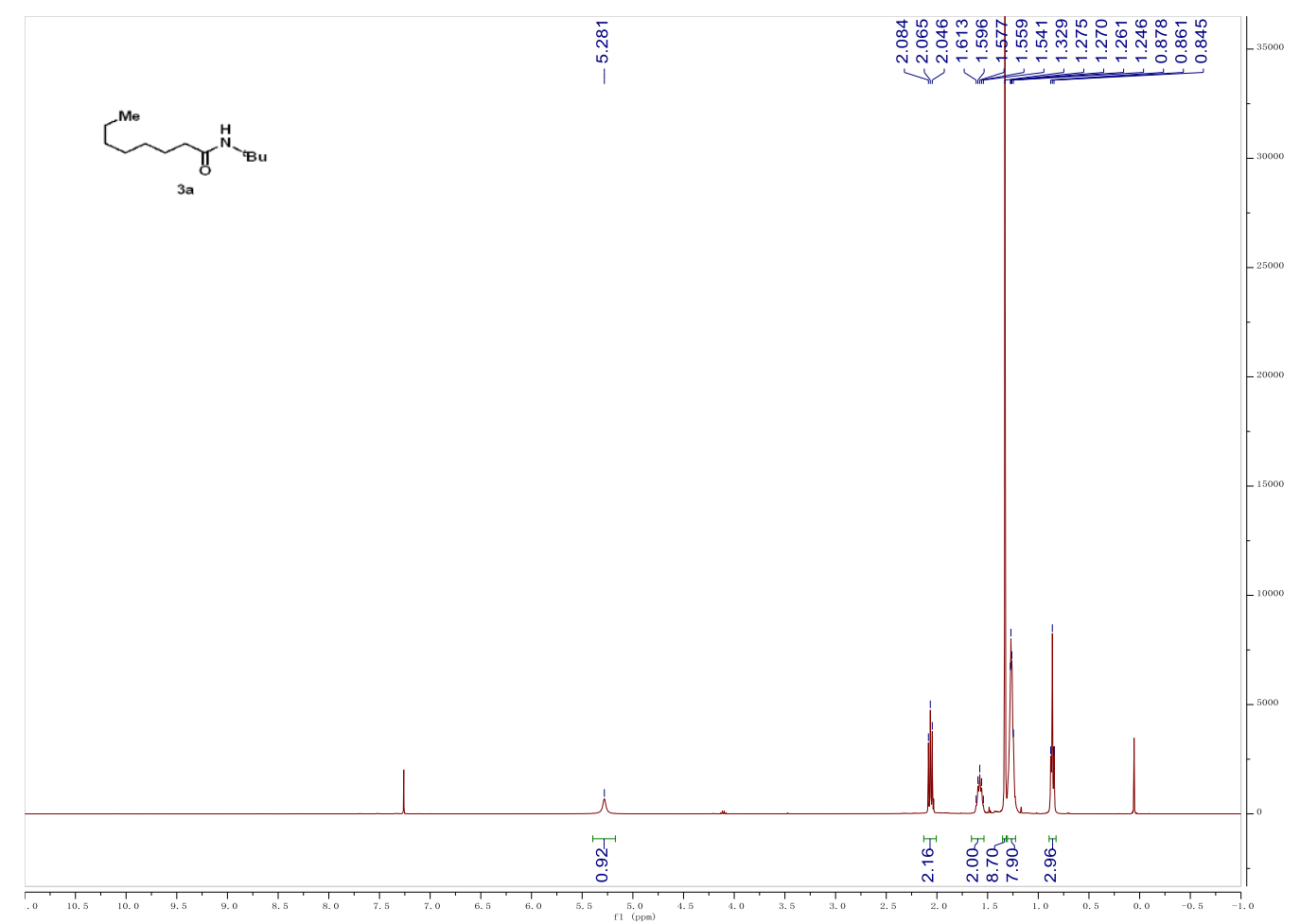

${ }^{13} \mathrm{C}$ NMR-spectrum(100 MHz, $\left.\mathrm{CDCl}_{3}\right)$ of 3a

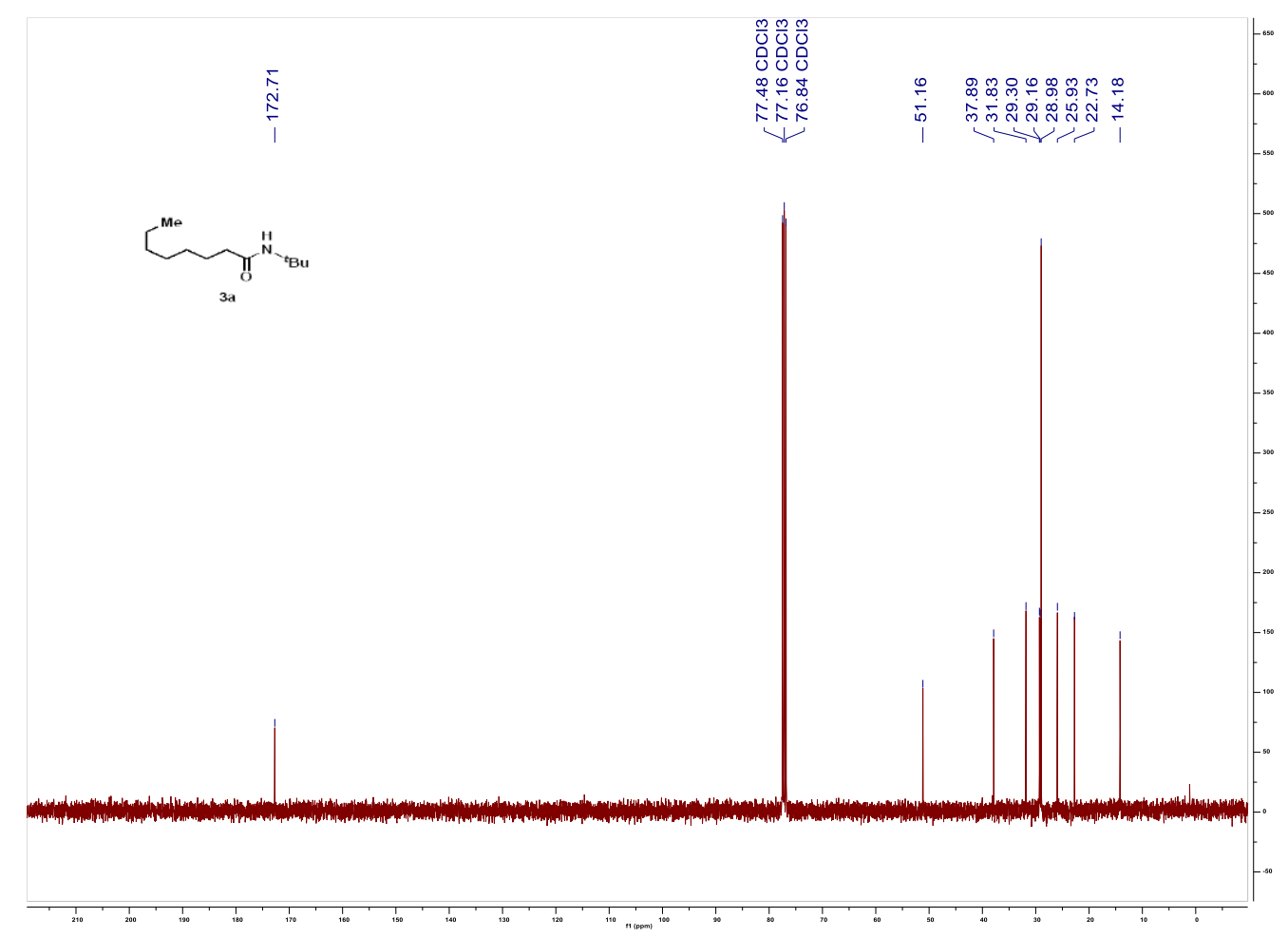


${ }^{1} \mathrm{H}$ NMR-spectrum(400 $\left.\mathrm{MHz}, \mathrm{CDCl}_{3}\right)$ of $3 \mathrm{~b}$

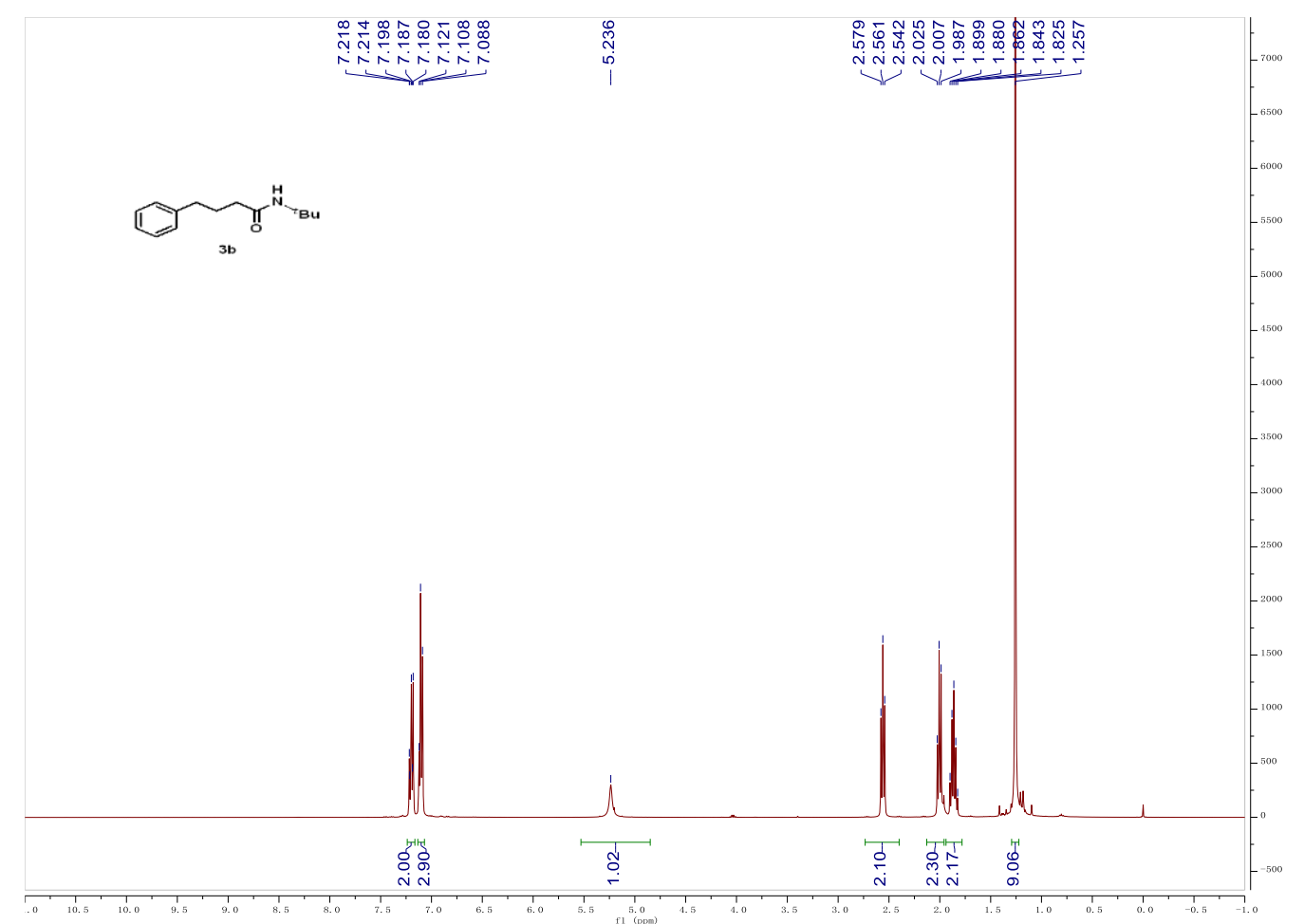

${ }^{13} \mathrm{C}$ NMR-spectrum(100 MHz, $\left.\mathrm{CDCl}_{3}\right)$ of $3 \mathrm{~b}$

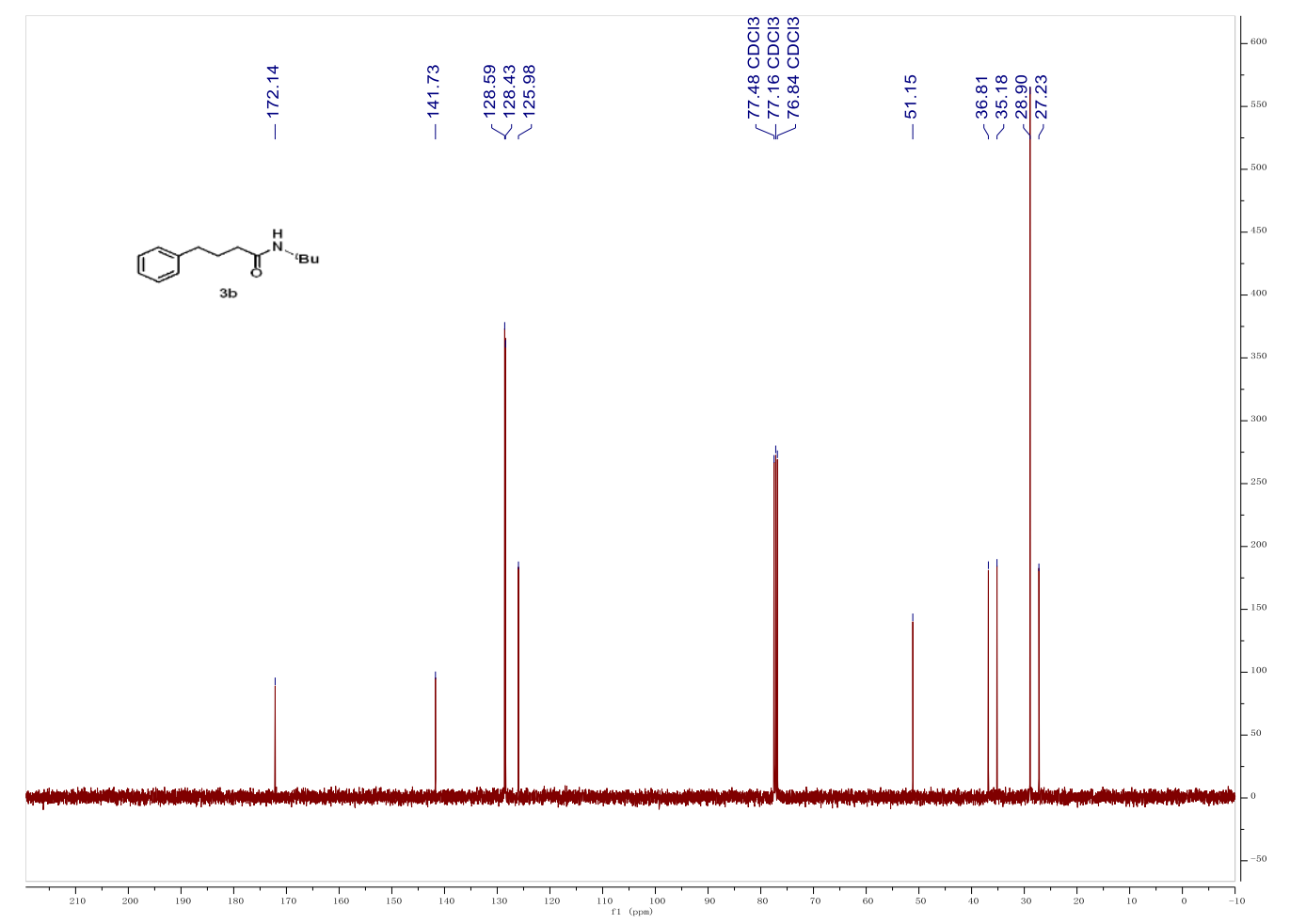


${ }^{1} \mathrm{H}$ NMR-spectrum(400 $\left.\mathrm{MHz}, \mathrm{CDCl}_{3}\right)$ of $3 \mathrm{c}$

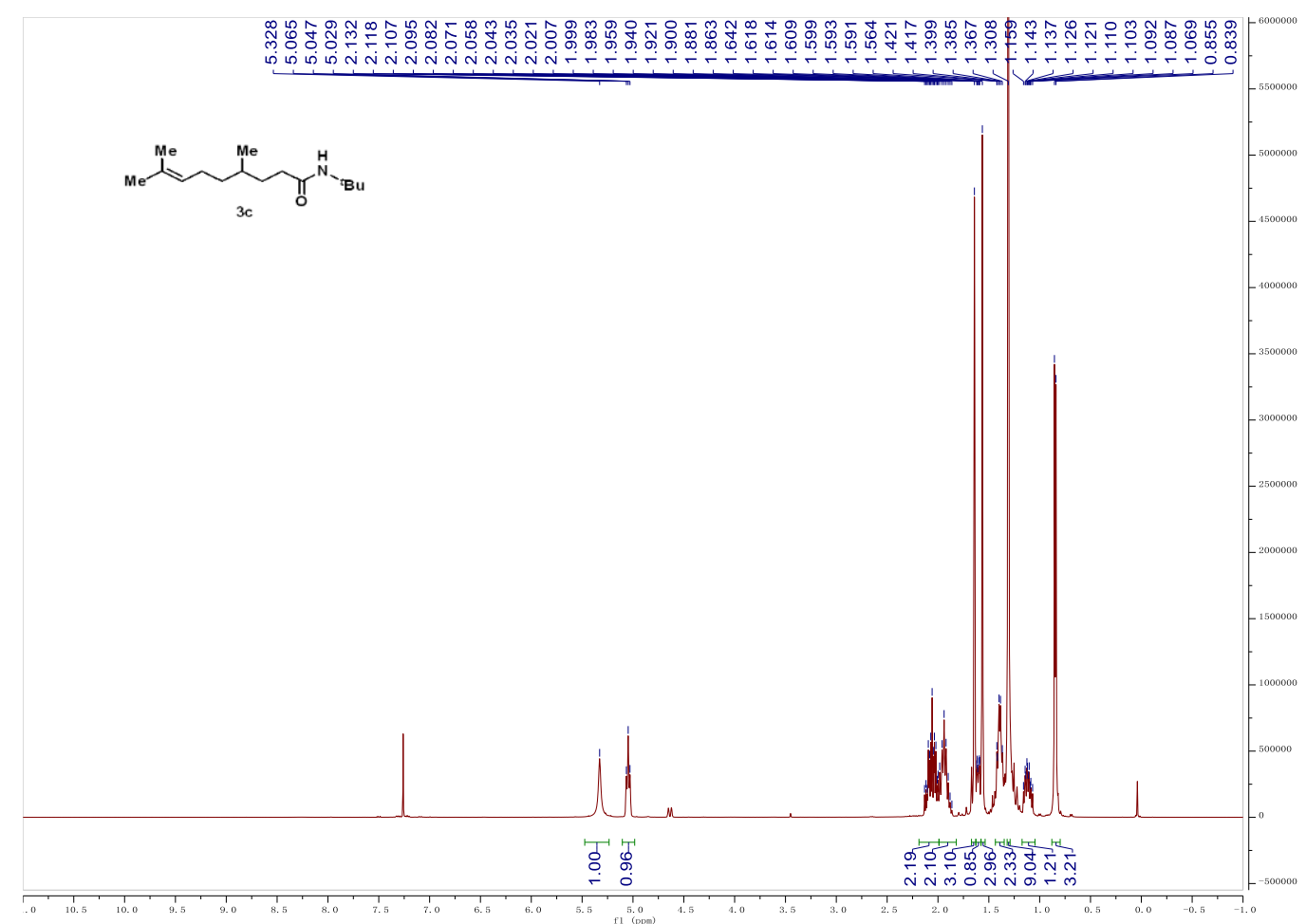

${ }^{13} \mathrm{C}$ NMR-spectrum(100 MHz, $\left.\mathrm{CDCl}_{3}\right)$ of $3 \mathrm{c}$

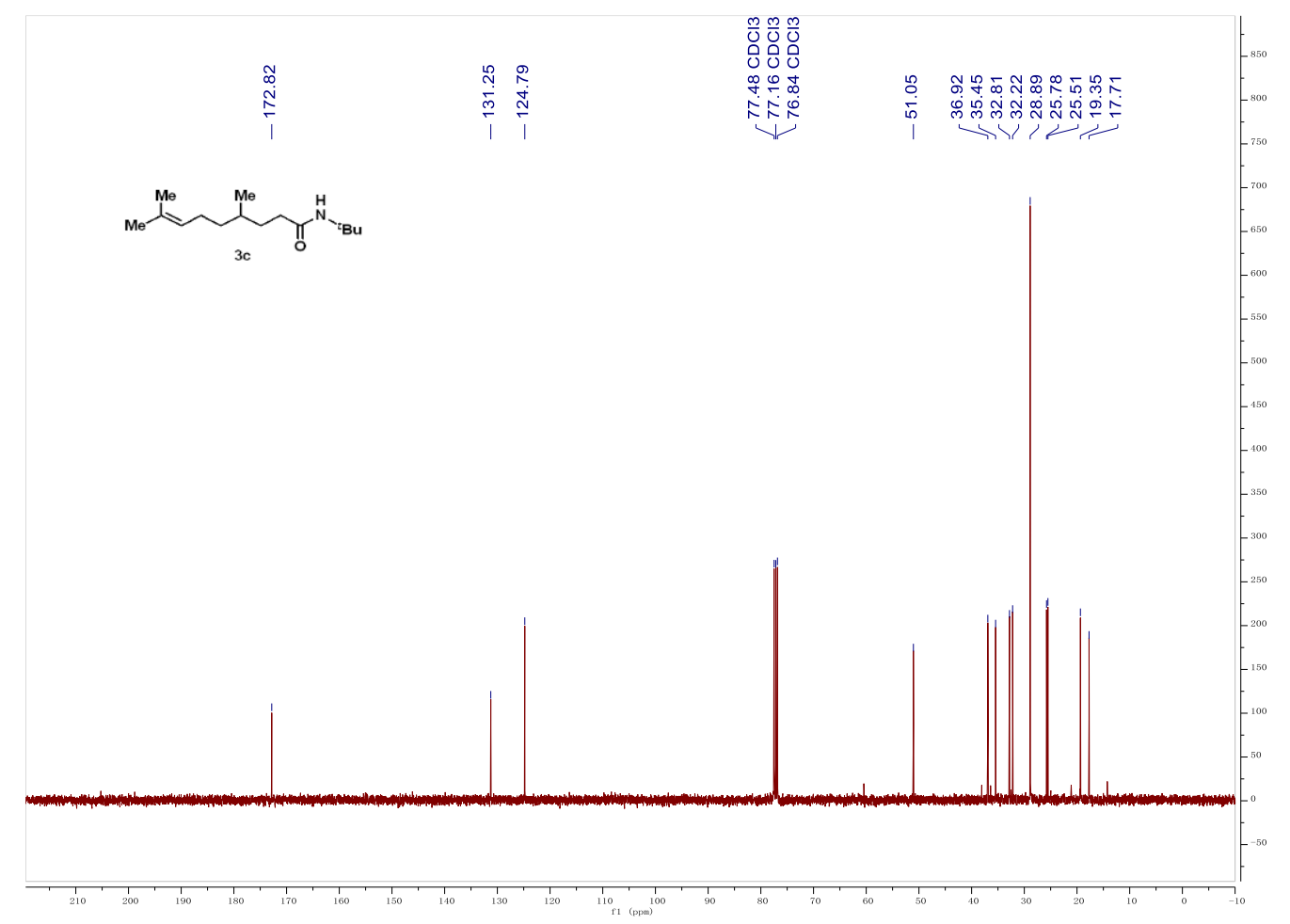


${ }^{1} \mathrm{H}$ NMR-spectrum(400 $\left.\mathrm{MHz}, \mathrm{CDCl}_{3}\right)$ of 3d

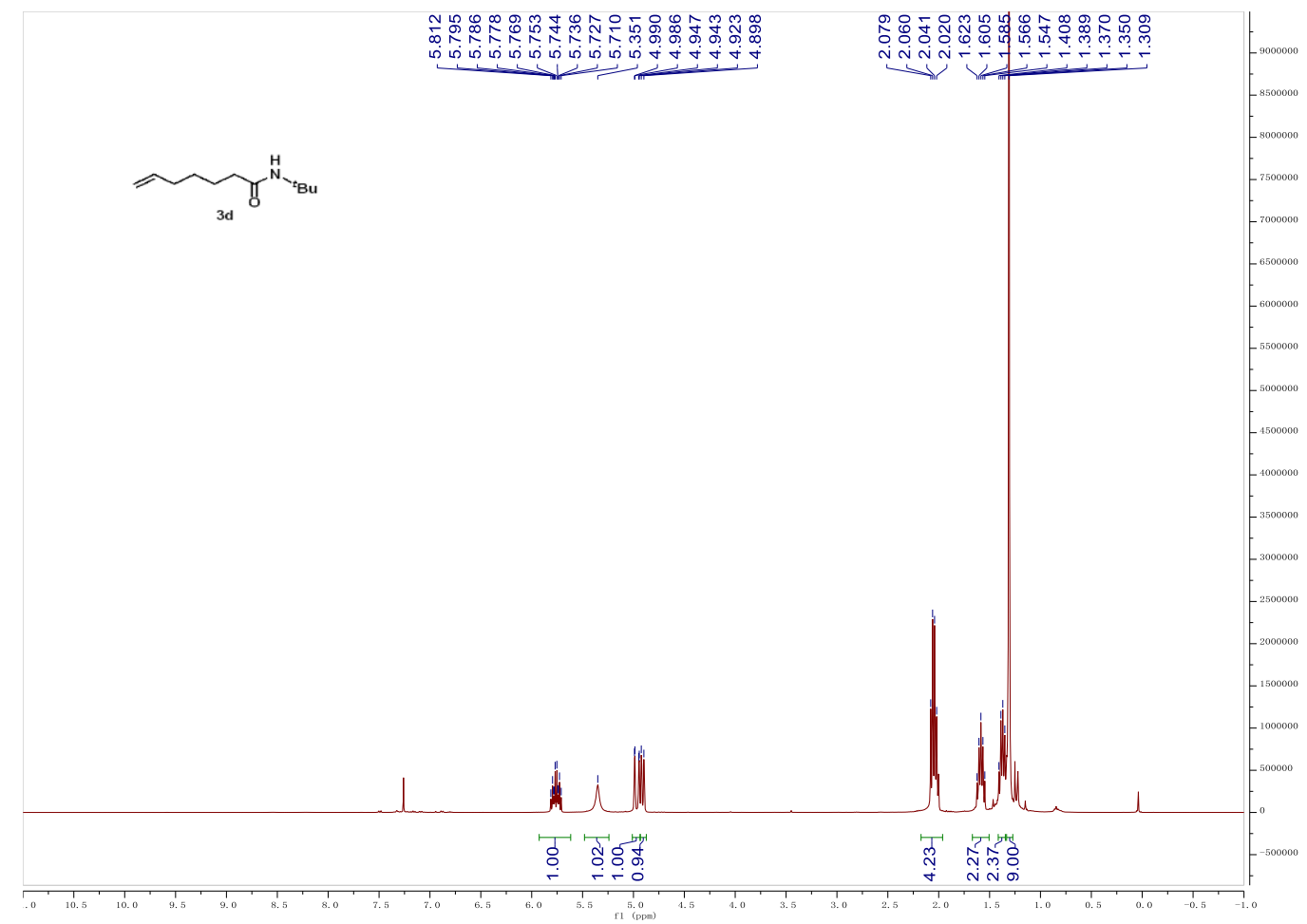

${ }^{13} \mathrm{C}$ NMR-spectrum(100 MHz, $\left.\mathrm{CDCl}_{3}\right)$ of 3d

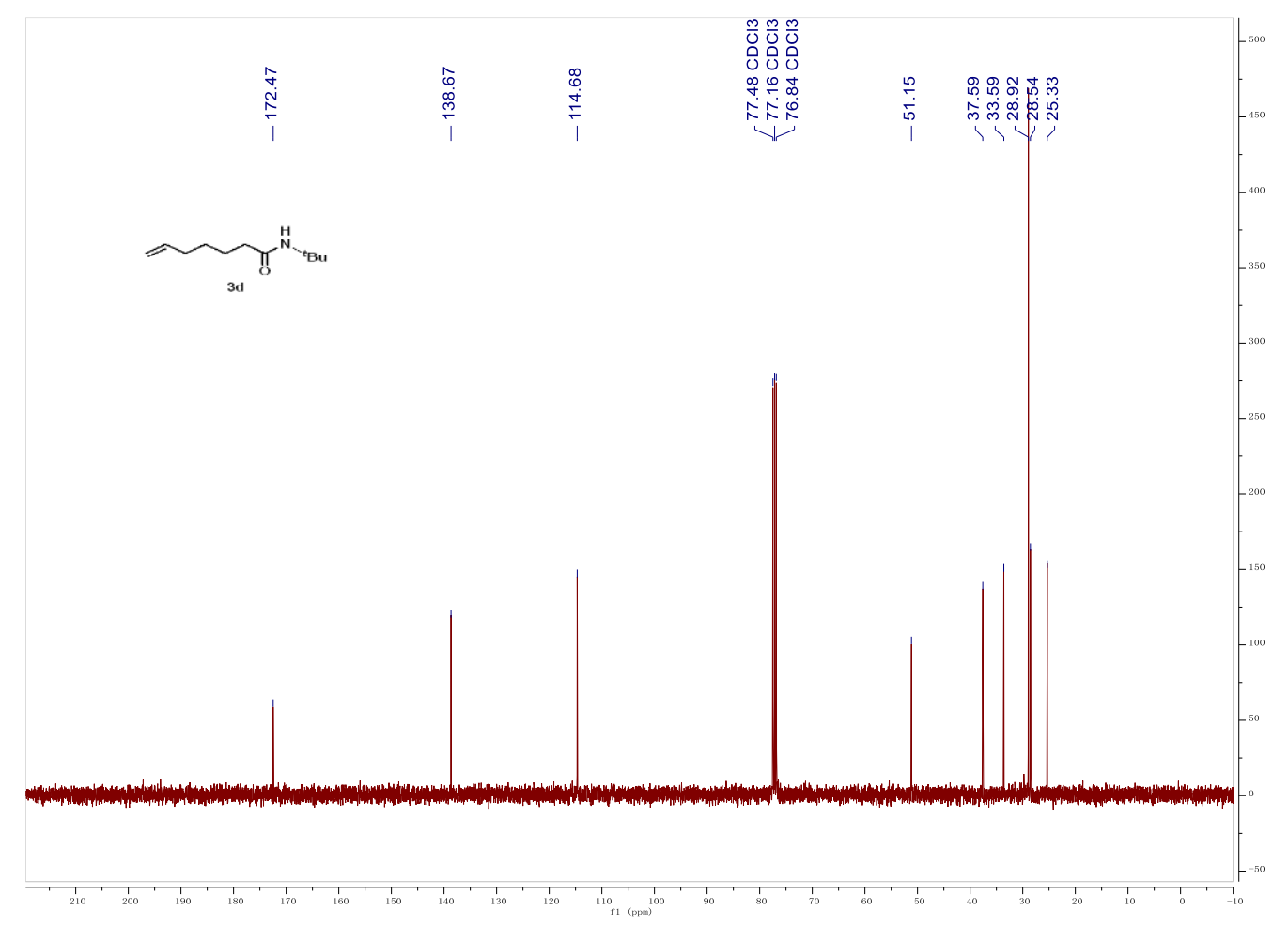


${ }^{1} \mathrm{H}$ NMR-spectrum(400 $\left.\mathrm{MHz}, \mathrm{CDCl}_{3}\right)$ of $3 \mathrm{e}$

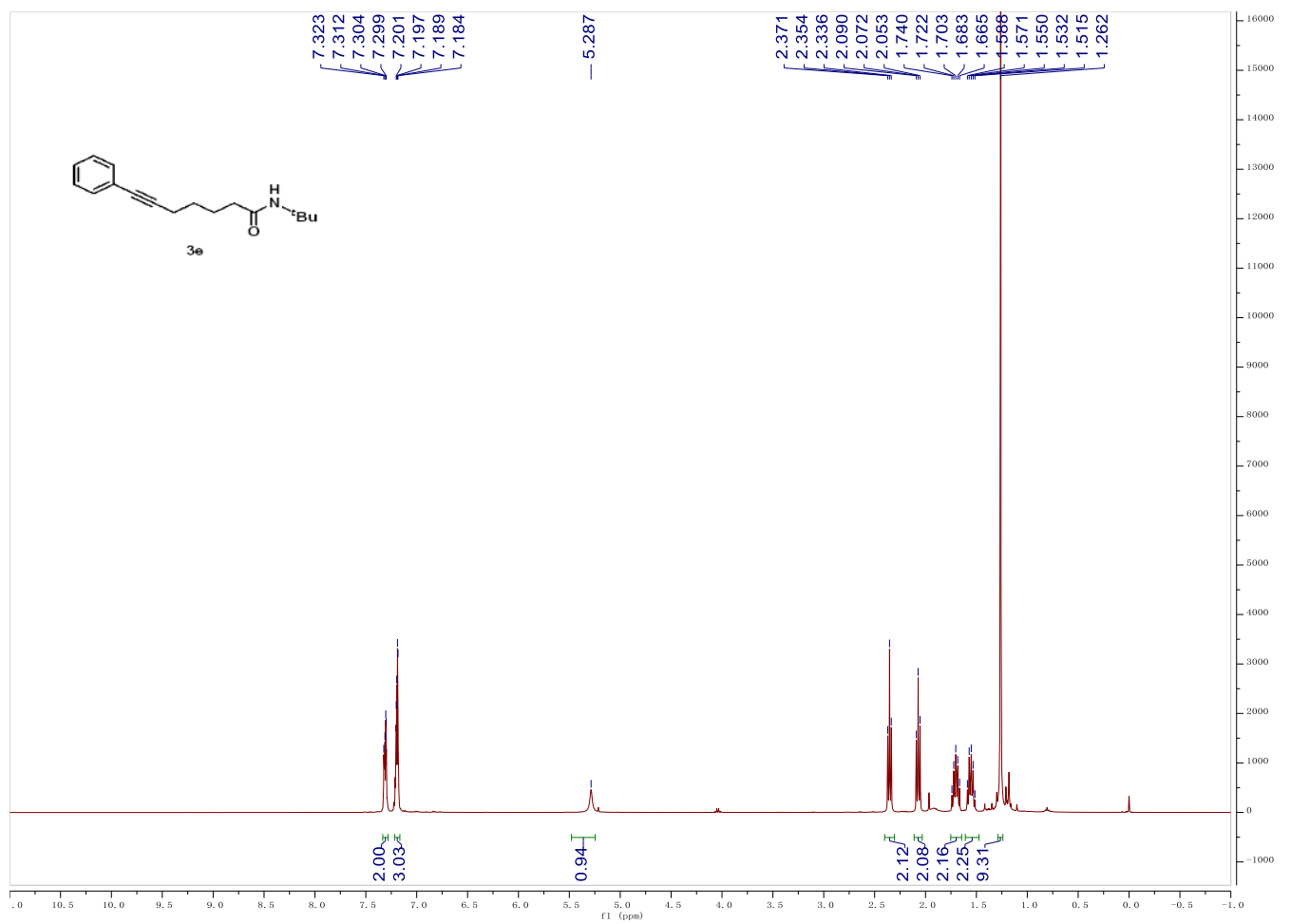

${ }^{13} \mathrm{C}$ NMR-spectrum(100 MHz, $\left.\mathrm{CDCl}_{3}\right)$ of $3 \mathrm{e}$

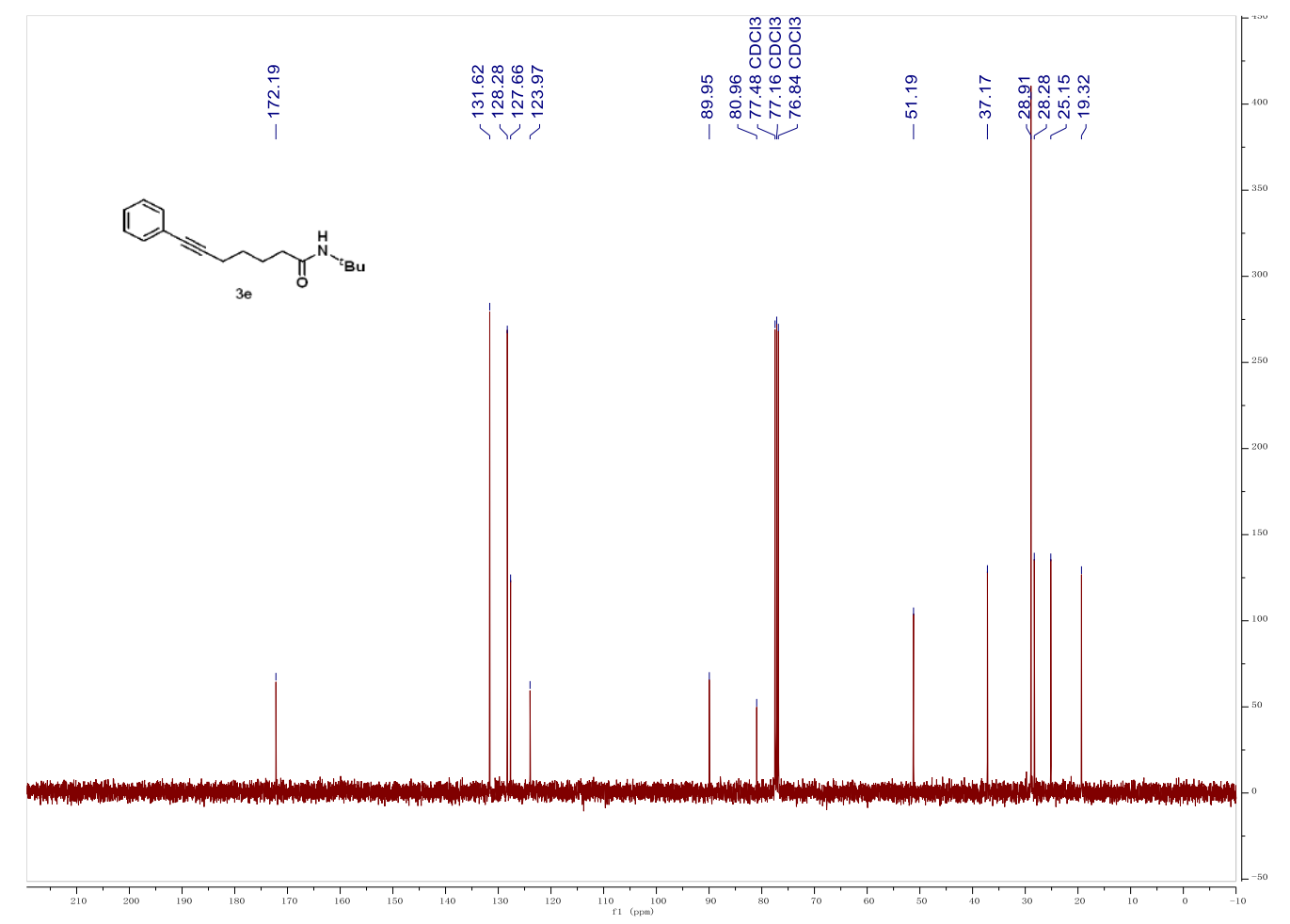


${ }^{1} \mathrm{H}$ NMR-spectrum(400 $\left.\mathrm{MHz}, \mathrm{CDCl}_{3}\right)$ of $3 \mathrm{f}$

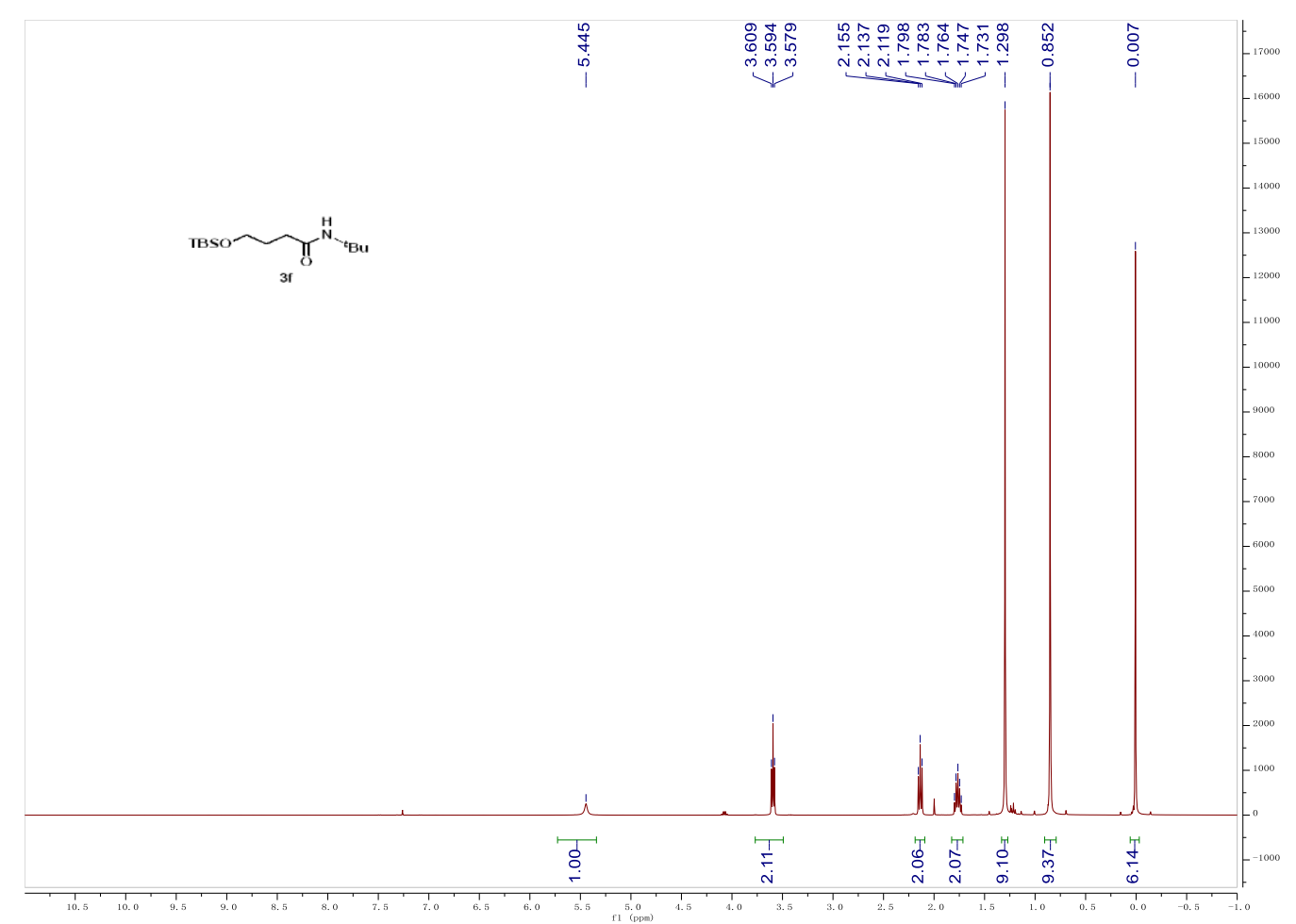

${ }^{13} \mathrm{C}$ NMR-spectrum(100 MHz, $\left.\mathrm{CDCl}_{3}\right)$ of $3 \mathrm{f}$

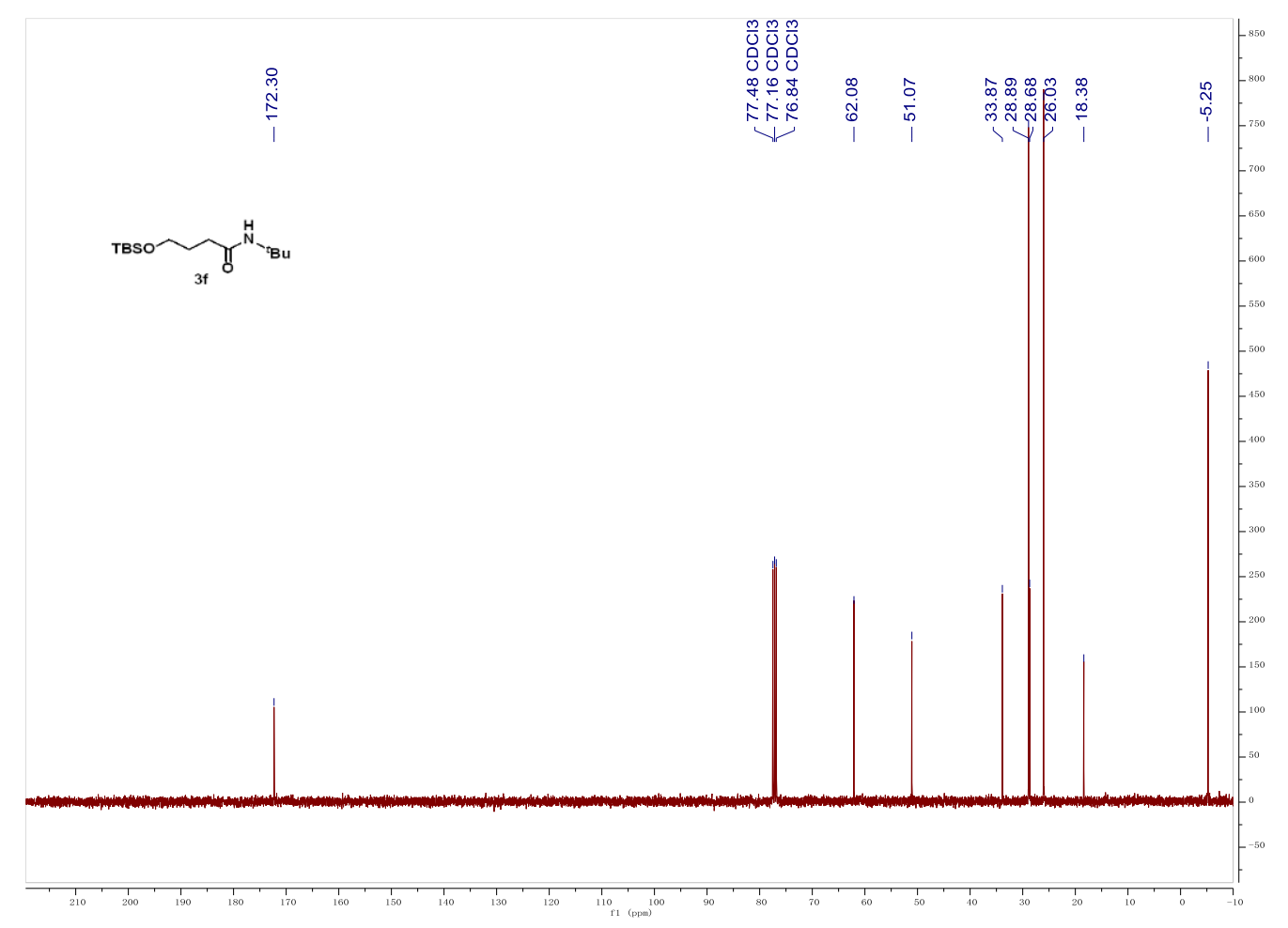


${ }^{1} \mathrm{H}$ NMR-spectrum(400 $\left.\mathrm{MHz}, \mathrm{CDCl}_{3}\right)$ of $3 \mathrm{~g}$

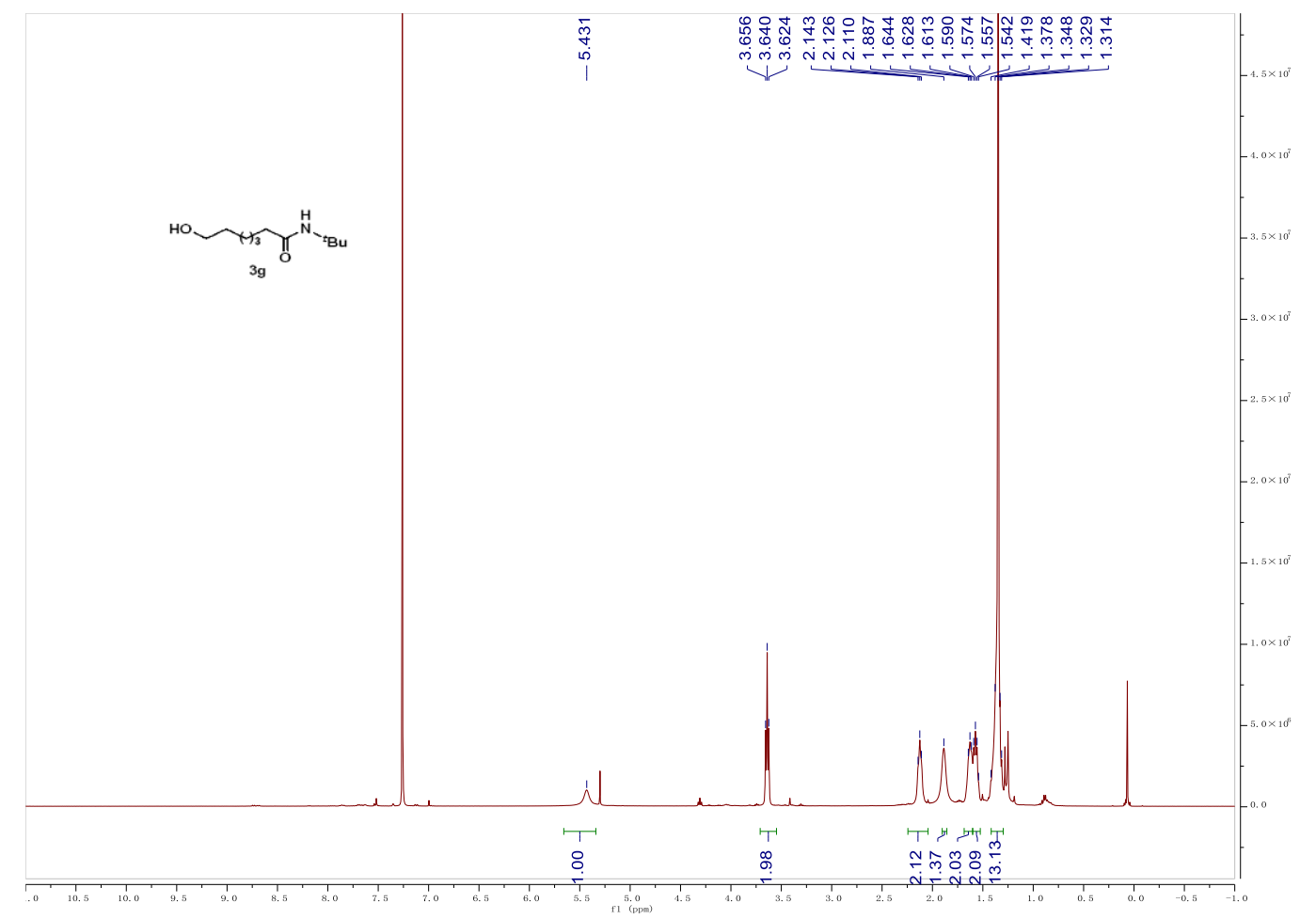

${ }^{13} \mathrm{C}$ NMR-spectrum(100 MHz, $\left.\mathrm{CDCl}_{3}\right)$ of $3 \mathrm{~g}$

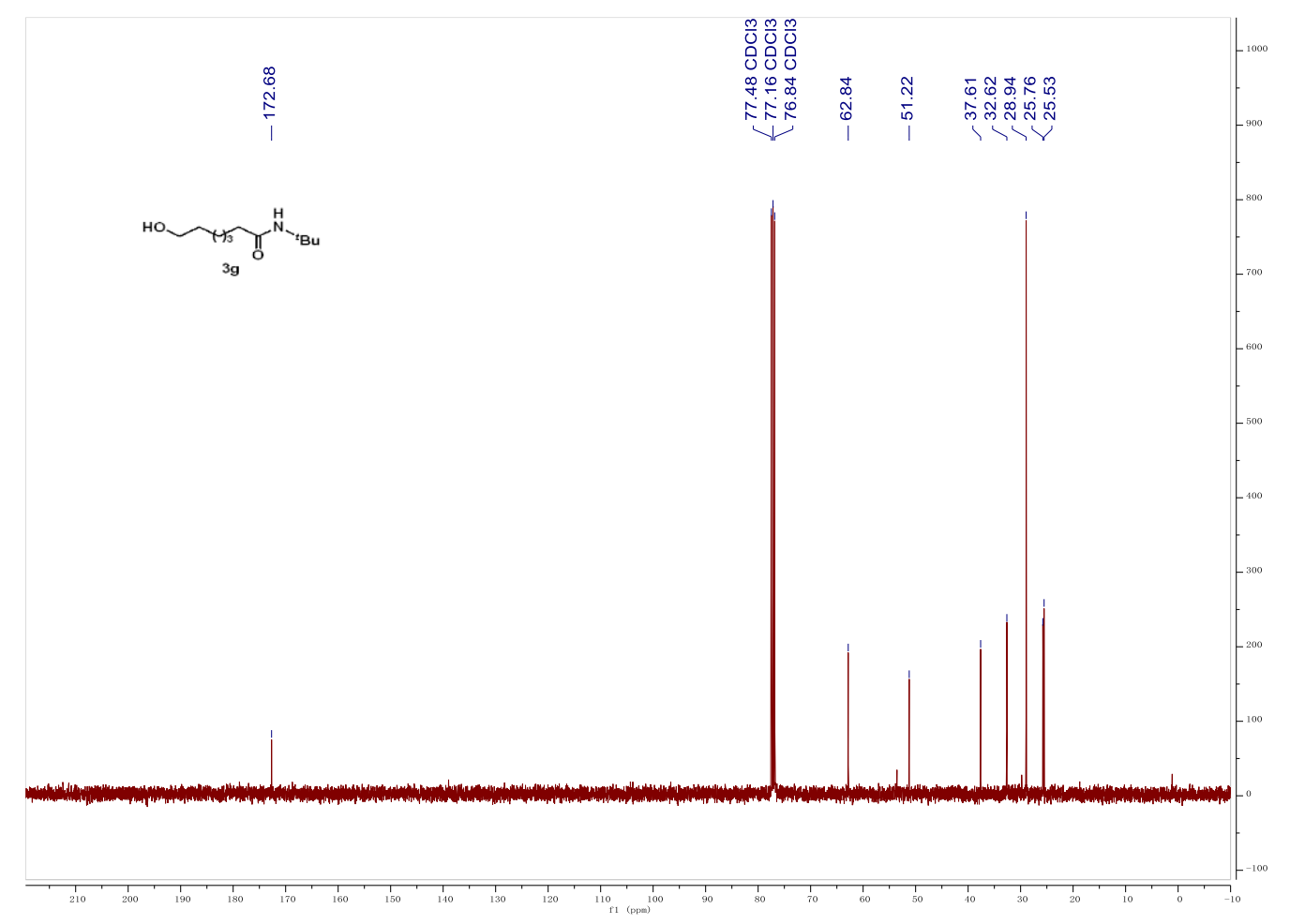


${ }^{1} \mathrm{H}$ NMR-spectrum(400 $\left.\mathrm{MHz}, \mathrm{CDCl}_{3}\right)$ of $3 \mathrm{~h}$

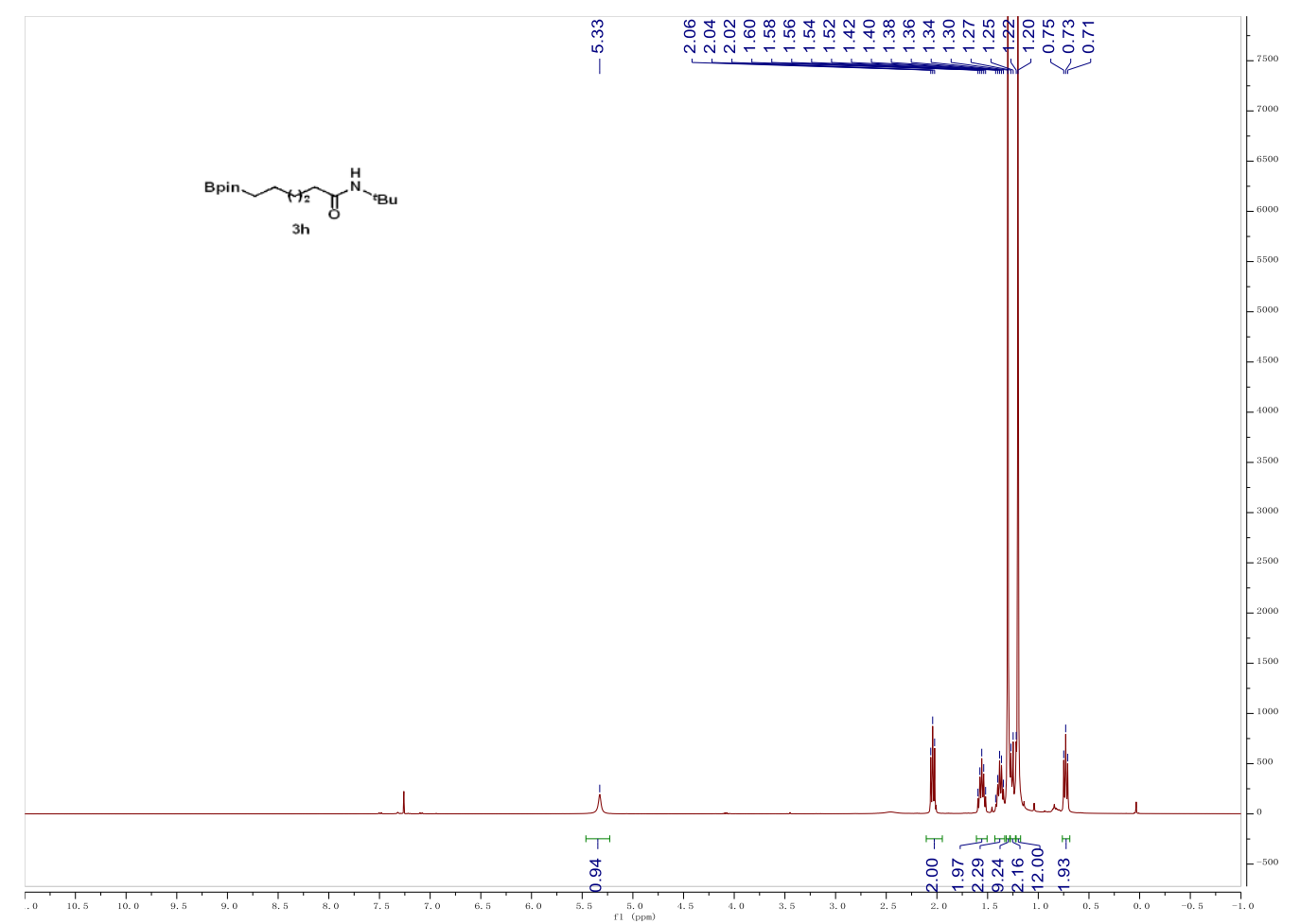

${ }^{13} \mathrm{C}$ NMR-spectrum(100 MHz, $\left.\mathrm{CDCl}_{3}\right)$ of $3 \mathrm{~h}$

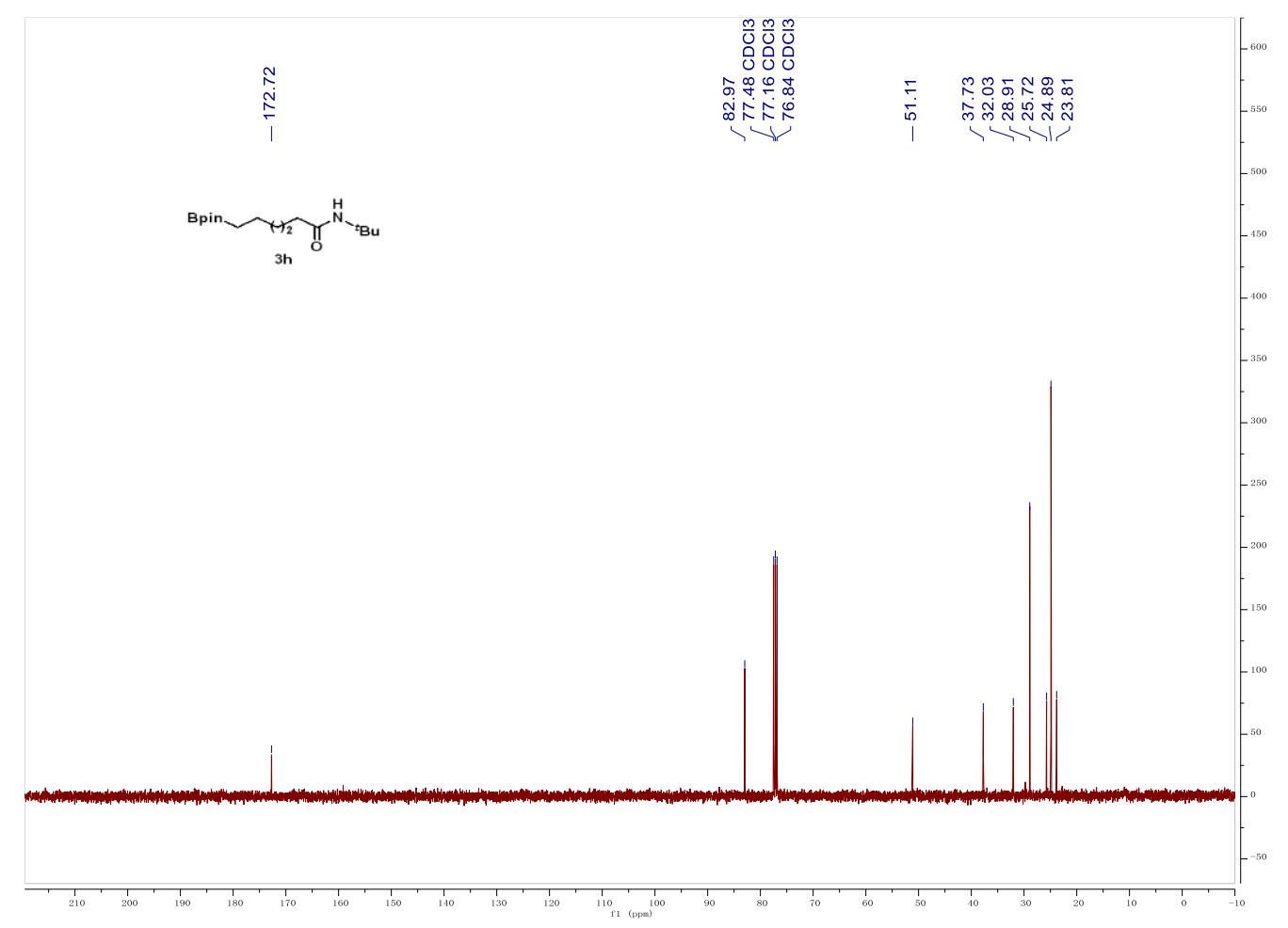


${ }^{1} \mathrm{H}$ NMR-spectrum(400 $\left.\mathrm{MHz}, \mathrm{CDCl}_{3}\right)$ of $3 \mathrm{i}$

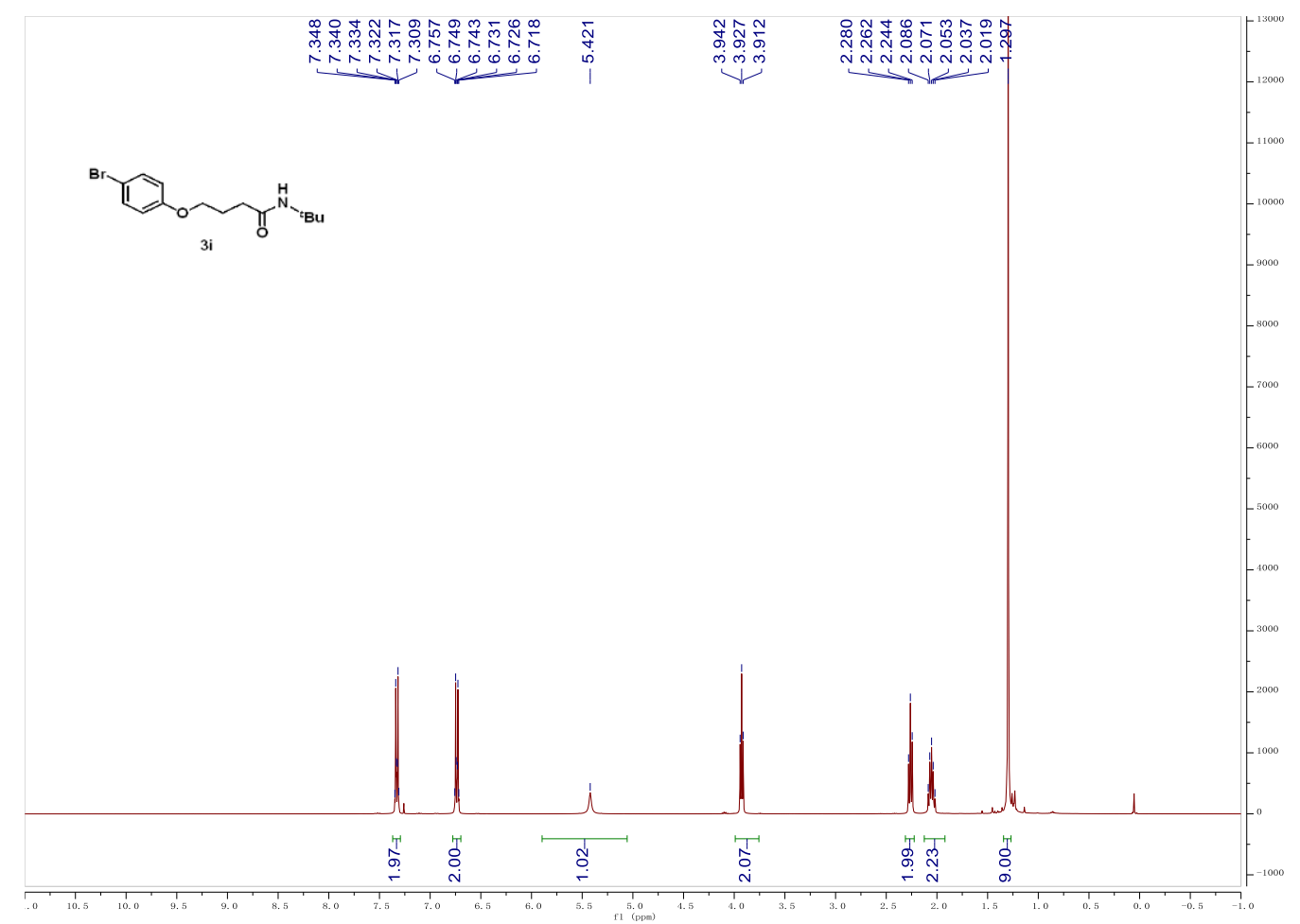

${ }^{13} \mathrm{C}$ NMR-spectrum(100 MHz, $\left.\mathrm{CDCl}_{3}\right)$ of $3 \mathrm{i}$

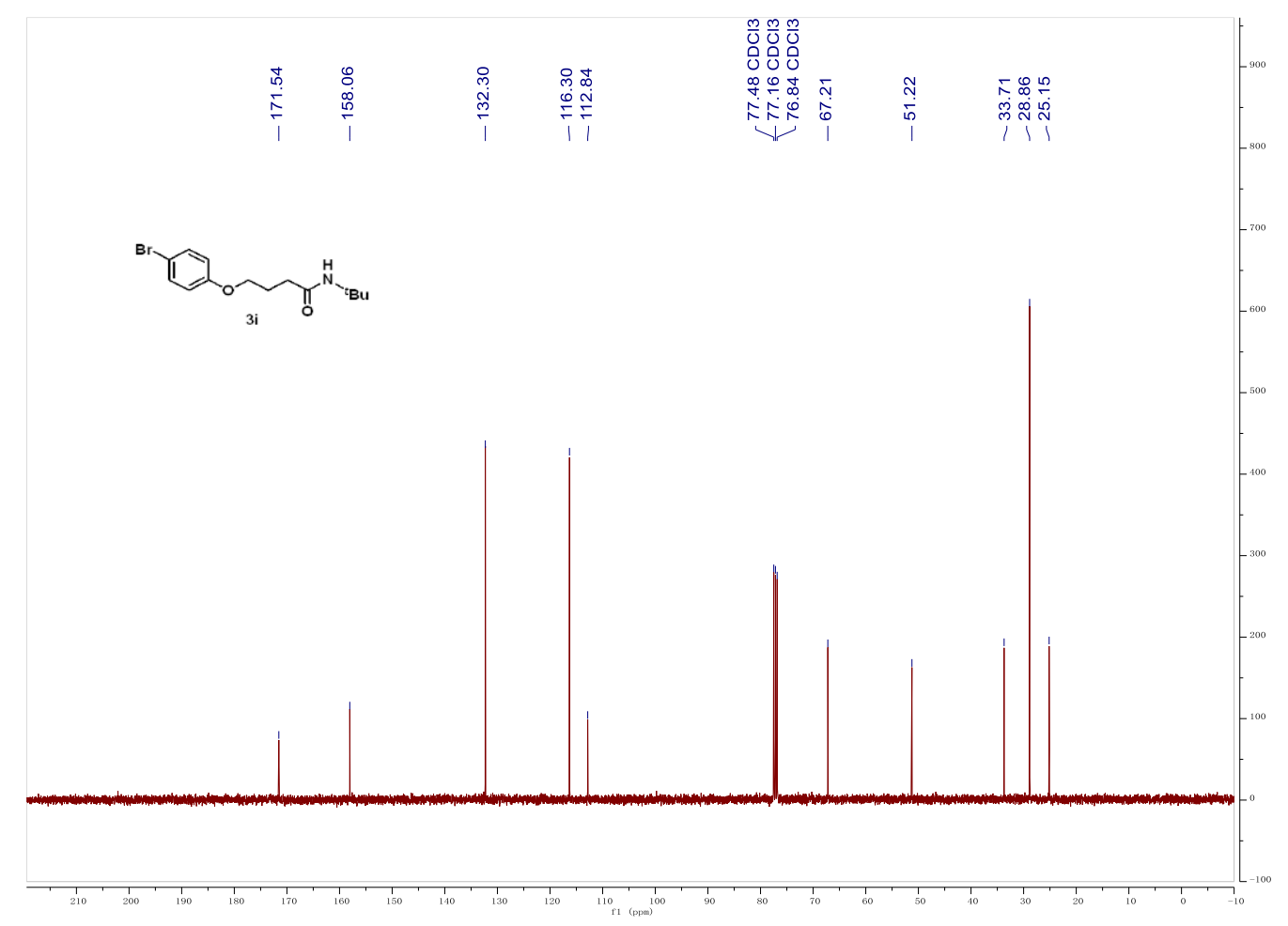


${ }^{1} \mathrm{H}$ NMR-spectrum(400 $\left.\mathrm{MHz}, \mathrm{CDCl}_{3}\right)$ of $3 \mathrm{j}$

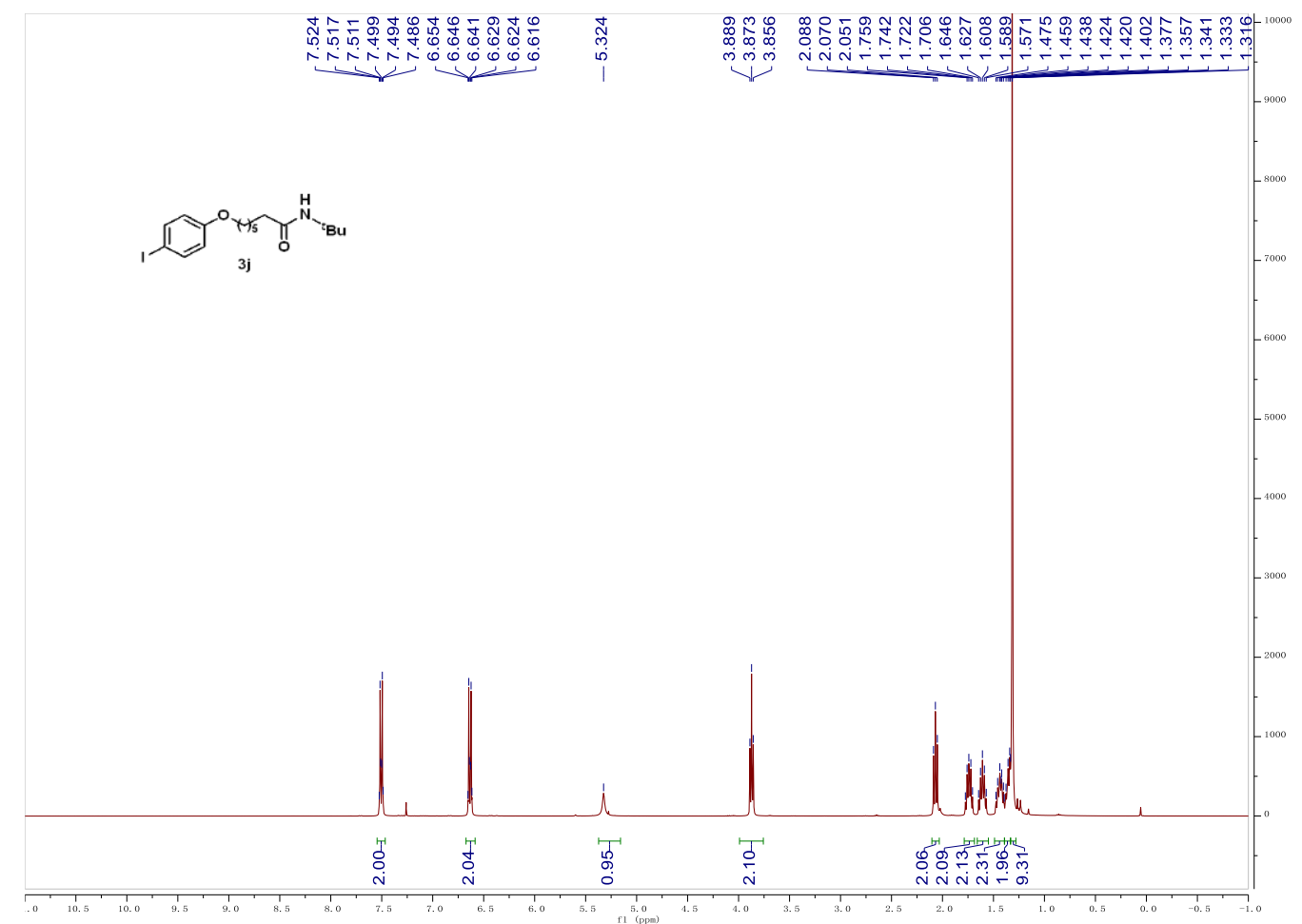

${ }^{13} \mathrm{C}$ NMR-spectrum(100 MHz, $\left.\mathrm{CDCl}_{3}\right)$ of $3 \mathrm{j}$

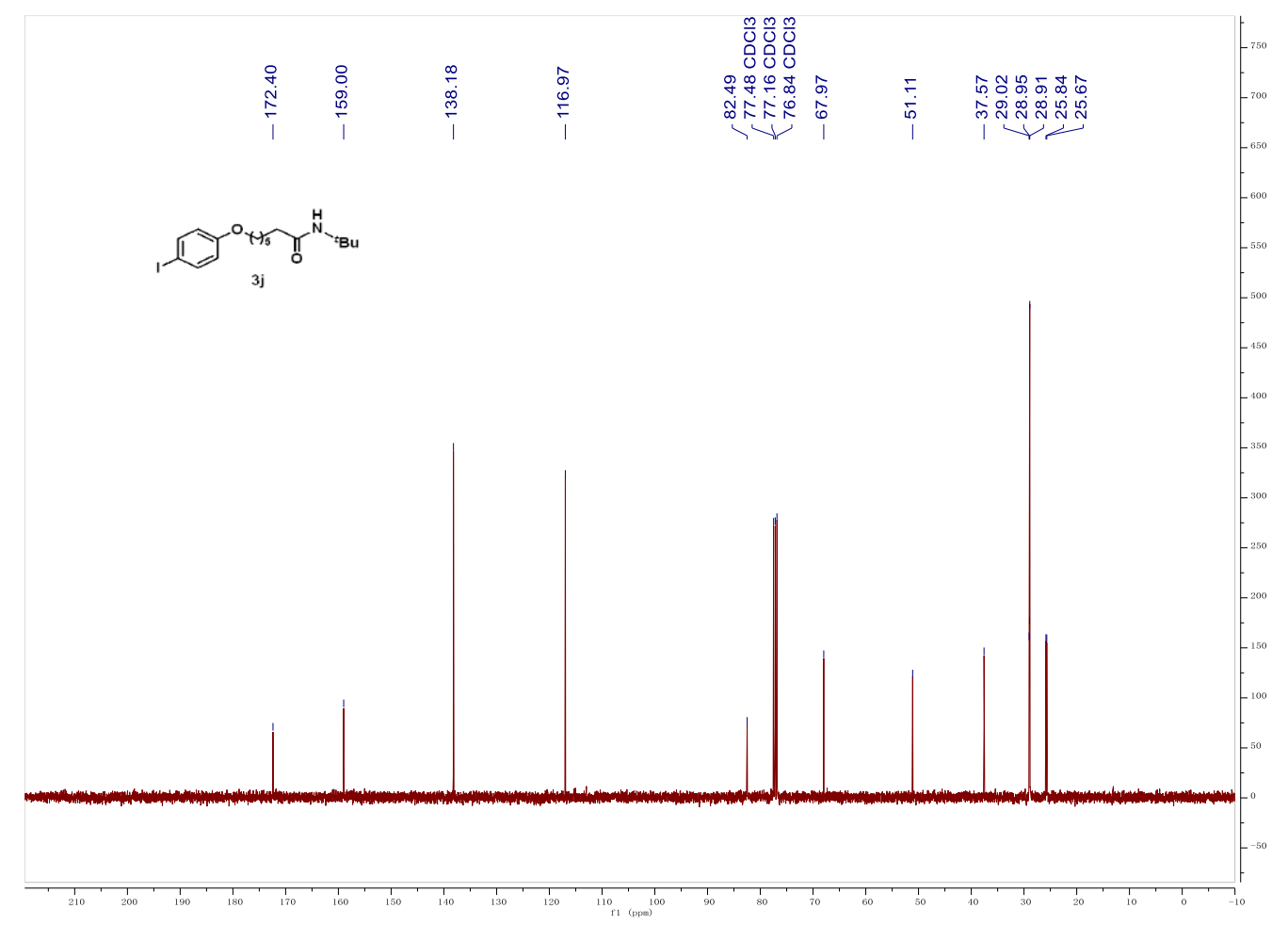


${ }^{1} \mathrm{H}$ NMR-spectrum(400 $\left.\mathrm{MHz}, \mathrm{CDCl}_{3}\right)$ of $3 \mathrm{k}$

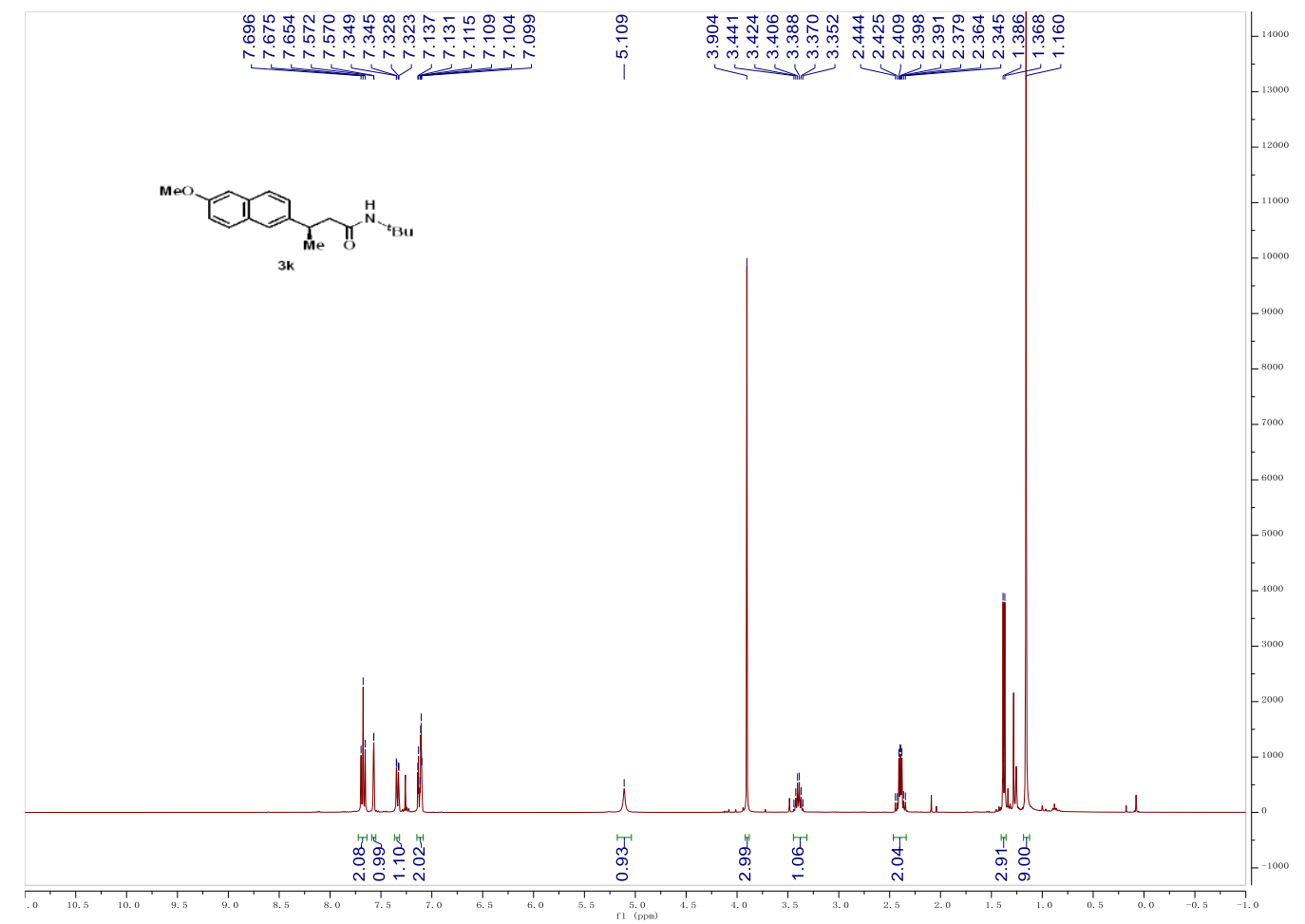

${ }^{13} \mathrm{C}$ NMR-spectrum(100 MHz, $\left.\mathrm{CDCl}_{3}\right)$ of $3 \mathrm{k}$

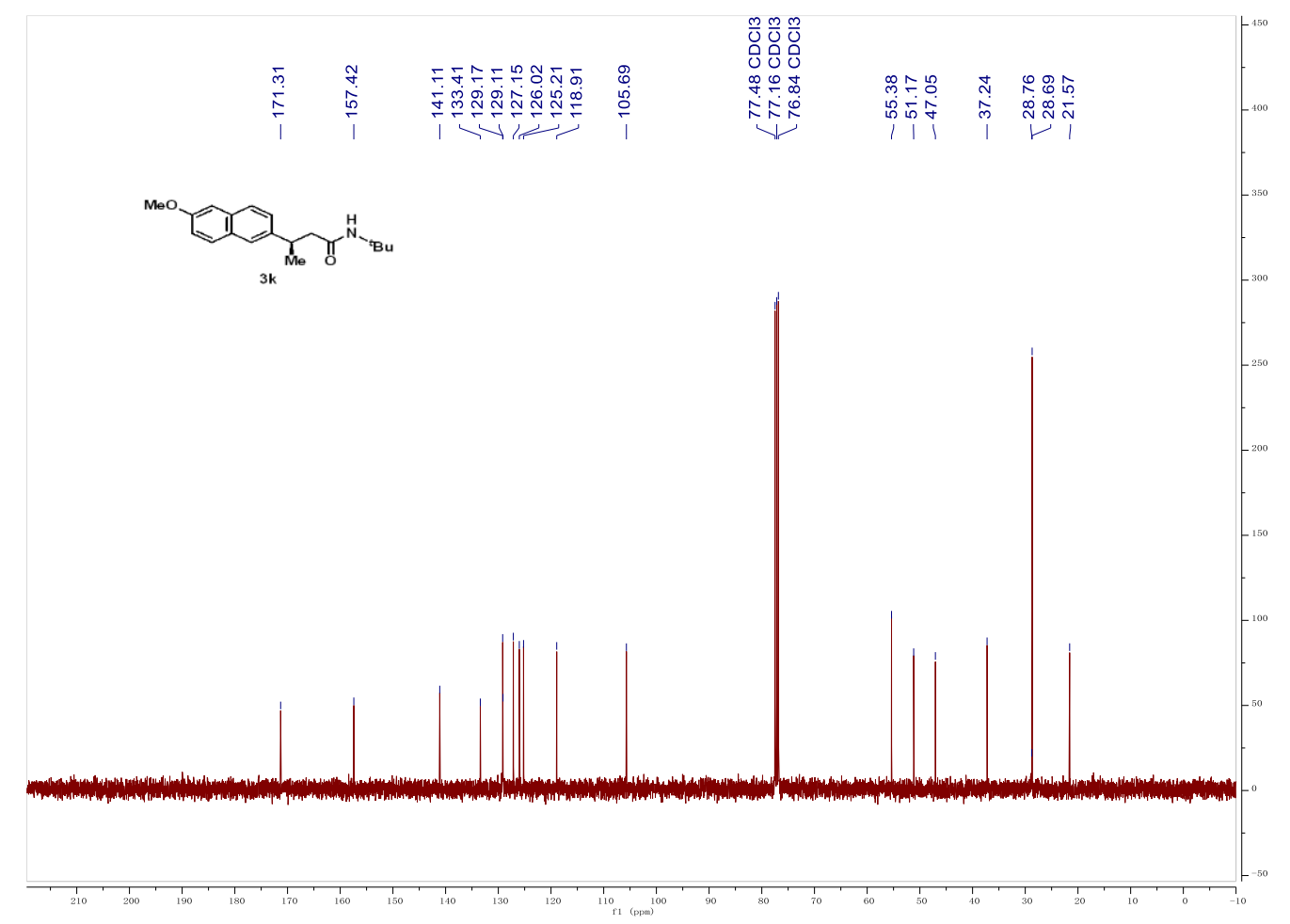


${ }^{1} \mathrm{H}$ NMR-spectrum(400 $\left.\mathrm{MHz}, \mathrm{CDCl}_{3}\right)$ of 31

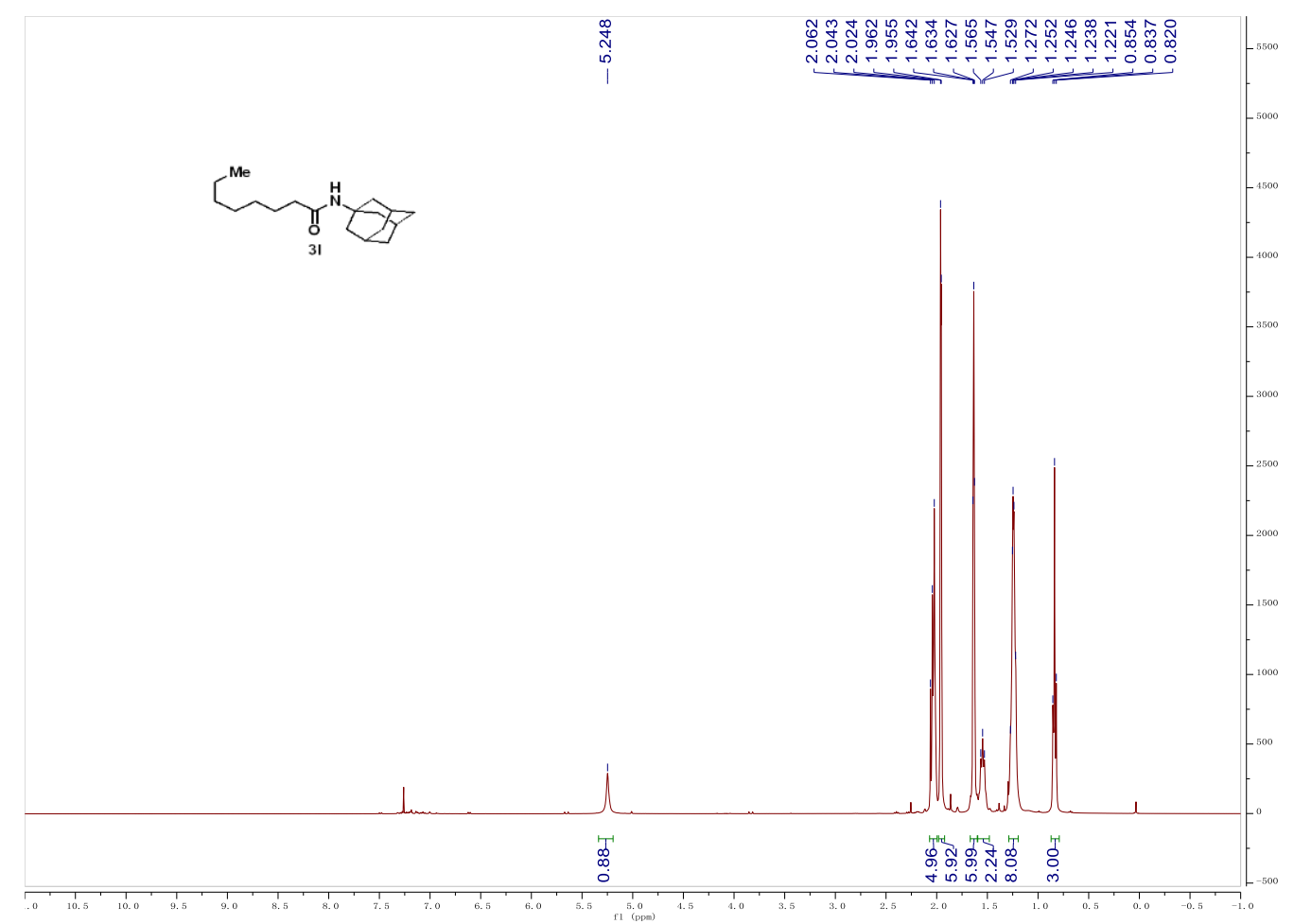

${ }^{13} \mathrm{C}$ NMR-spectrum(100 $\left.\mathrm{MHz}, \mathrm{CDCl}_{3}\right)$ of $3 \mathrm{I}$

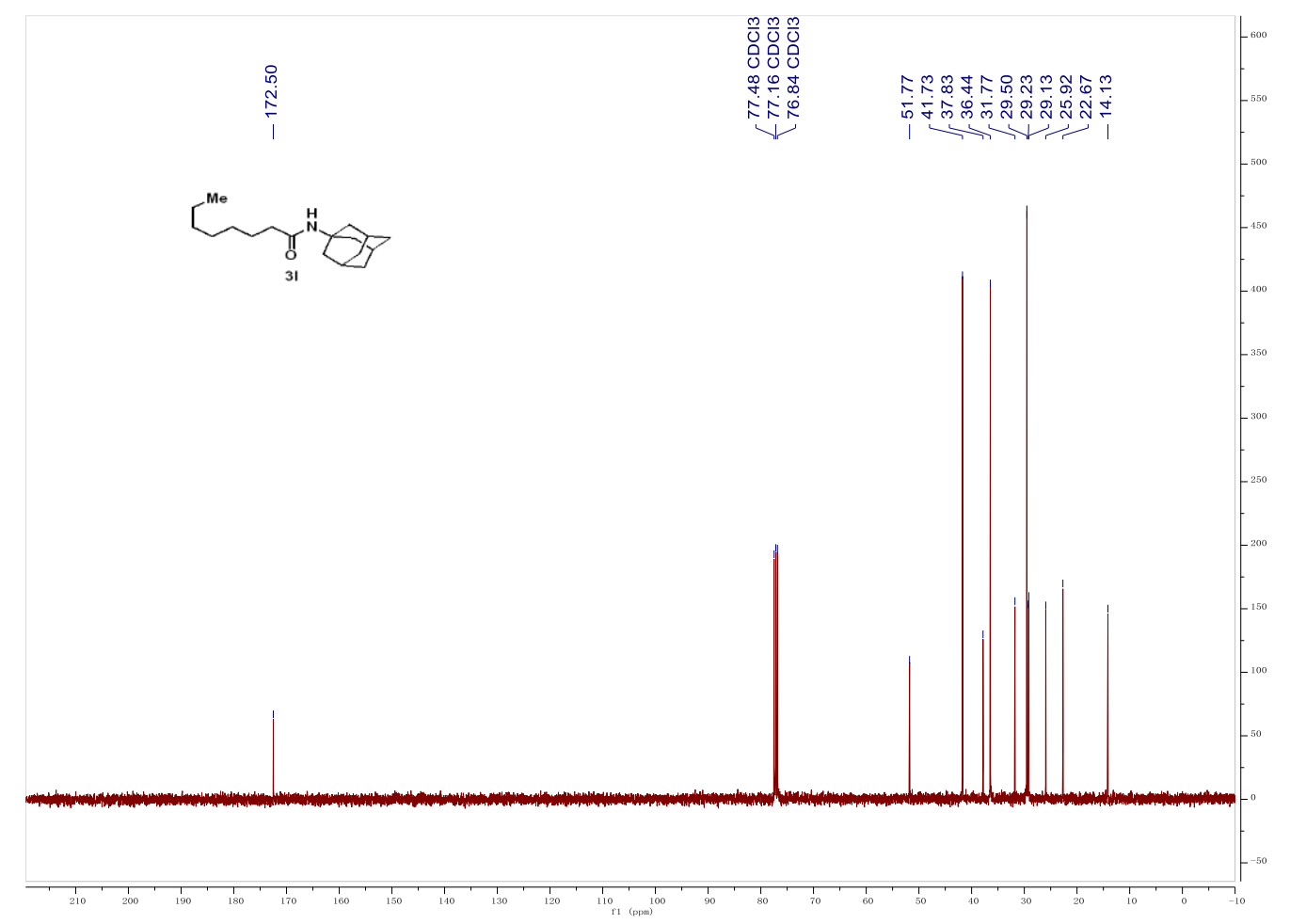


${ }^{1} \mathrm{H}$ NMR-spectrum(400 $\left.\mathrm{MHz}, \mathrm{CDCl}_{3}\right)$ of $3 \mathrm{~m}$

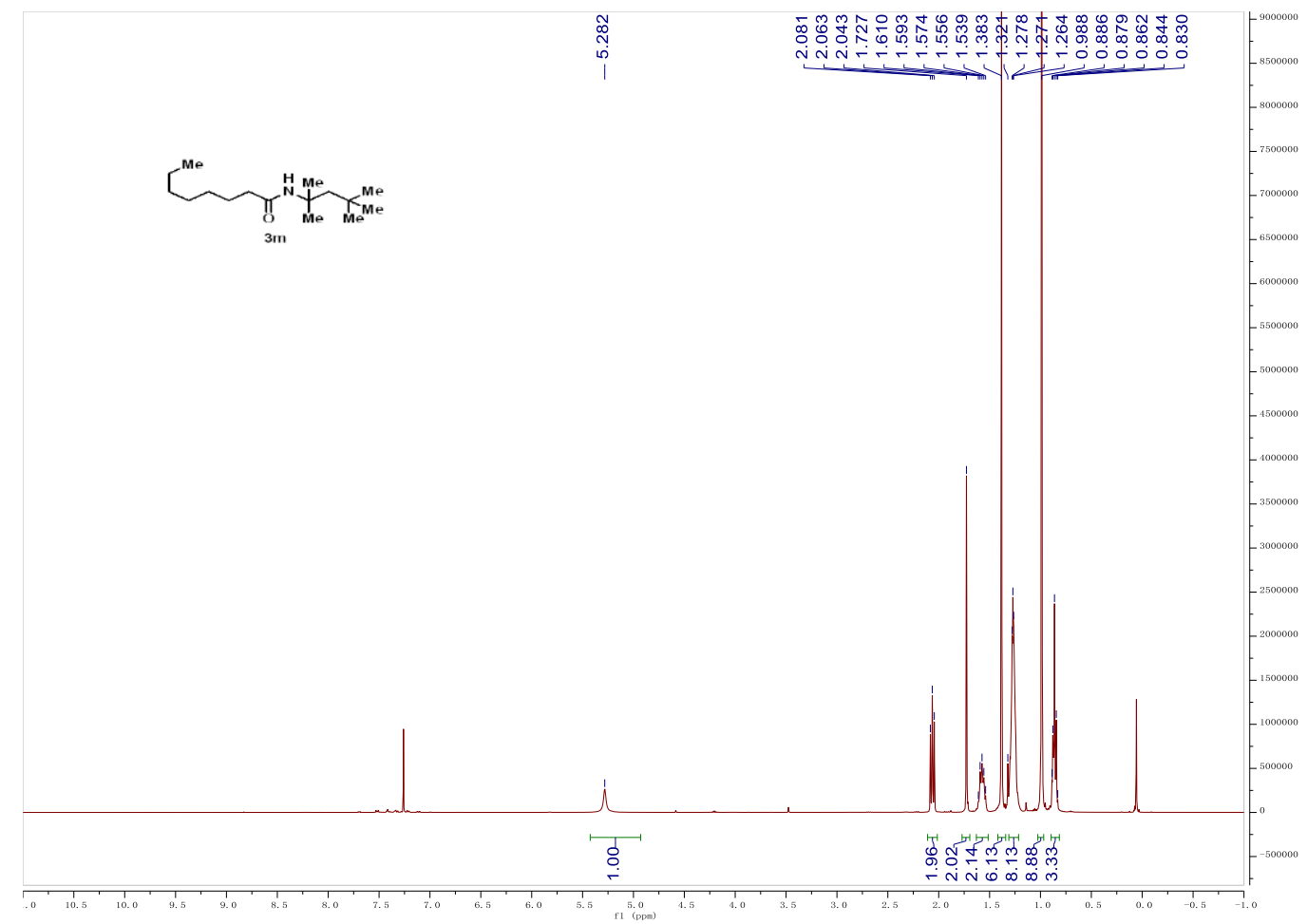

${ }^{13} \mathrm{C}$ NMR-spectrum(100 $\left.\mathrm{MHz}, \mathrm{CDCl}_{3}\right)$ of $3 \mathrm{~m}$

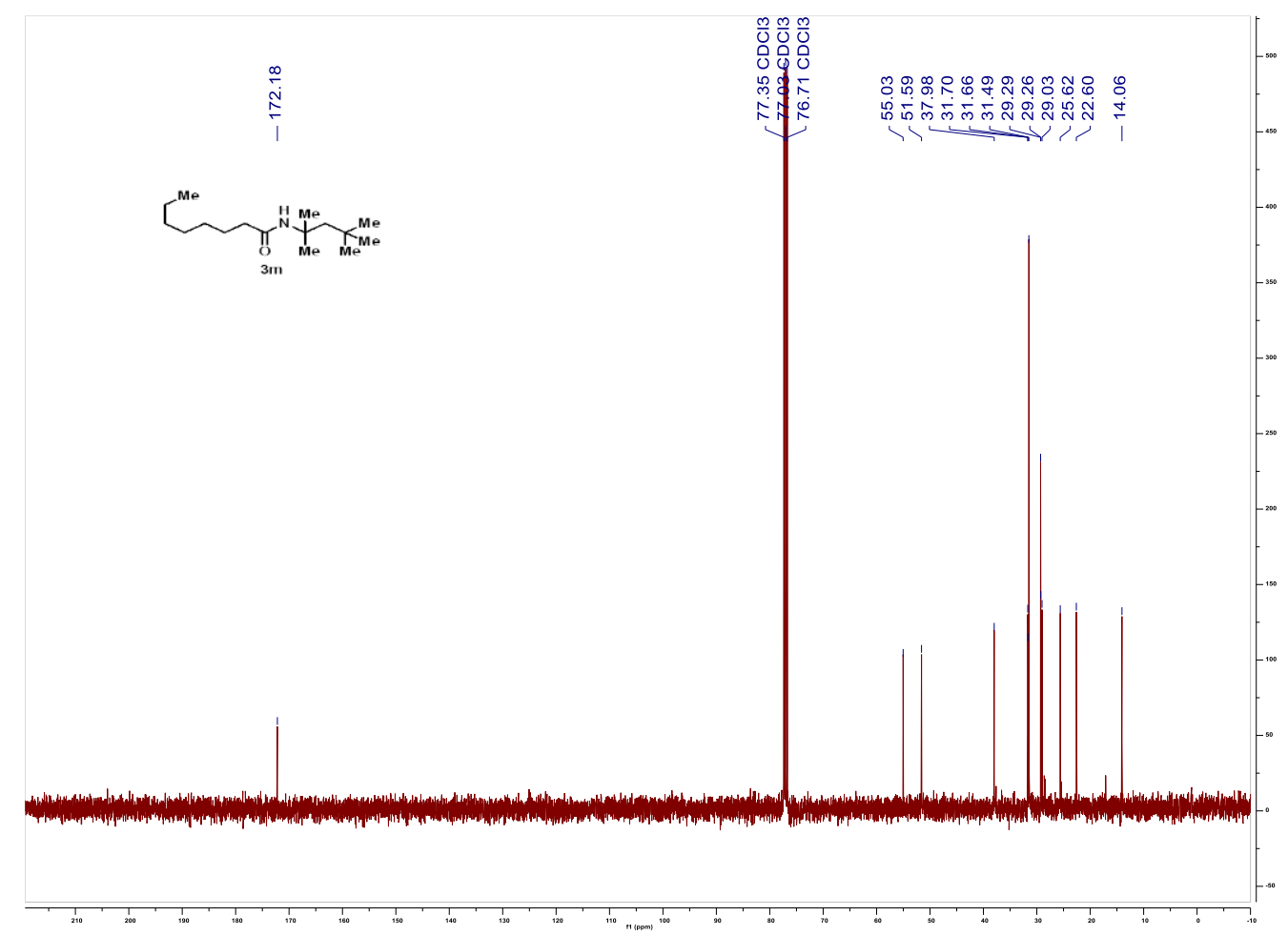


${ }^{1} \mathrm{H}$ NMR-spectrum(400 $\left.\mathrm{MHz}, \mathrm{CDCl}_{3}\right)$ of $3 \mathrm{n}$

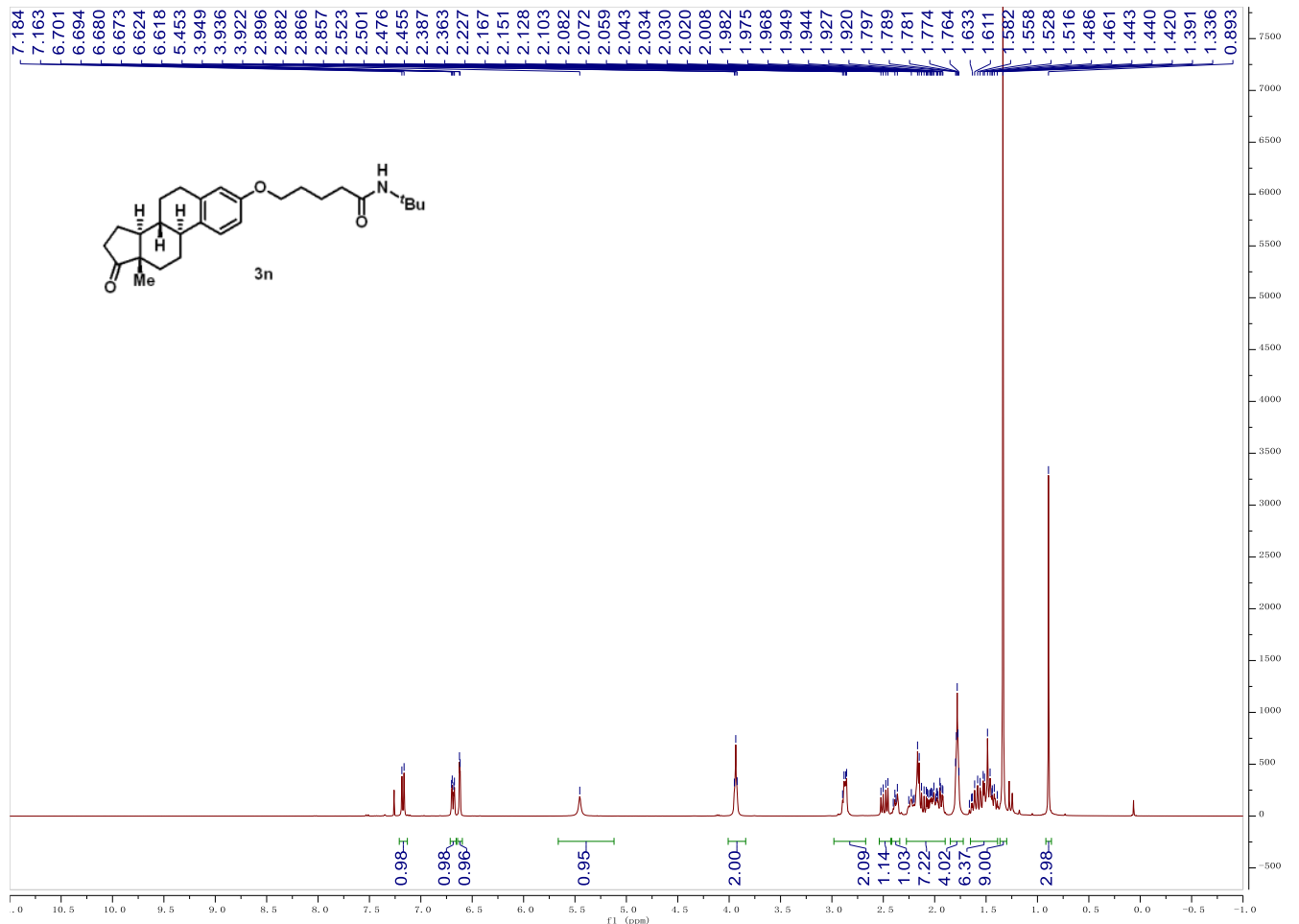

${ }^{13} \mathrm{C}$ NMR-spectrum(100 MHz, $\left.\mathrm{CDCl}_{3}\right)$ of 3n

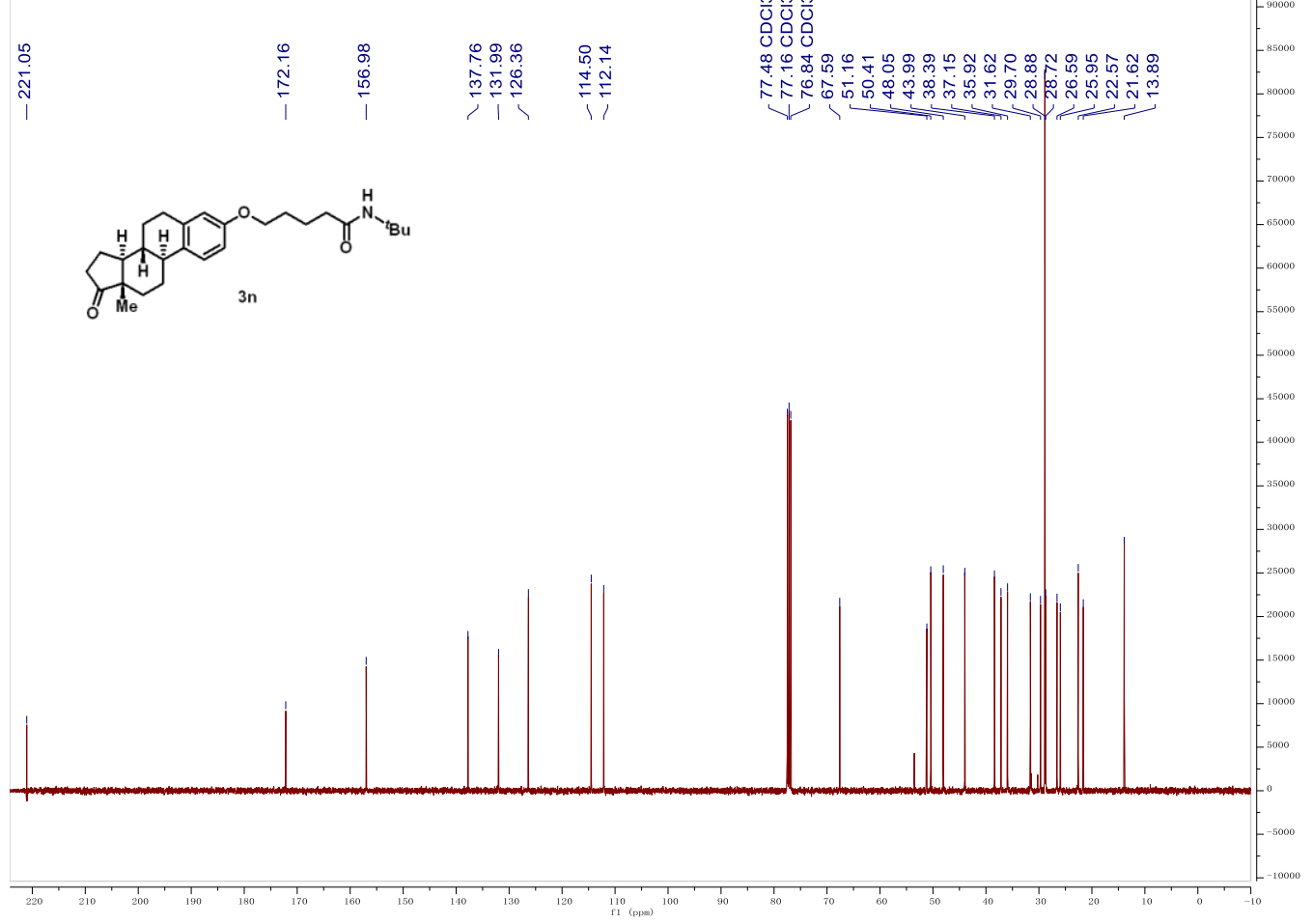


${ }^{1} \mathrm{H}$ NMR-spectrum(400 $\left.\mathrm{MHz}, \mathrm{CDCl}_{3}\right)$ of 30

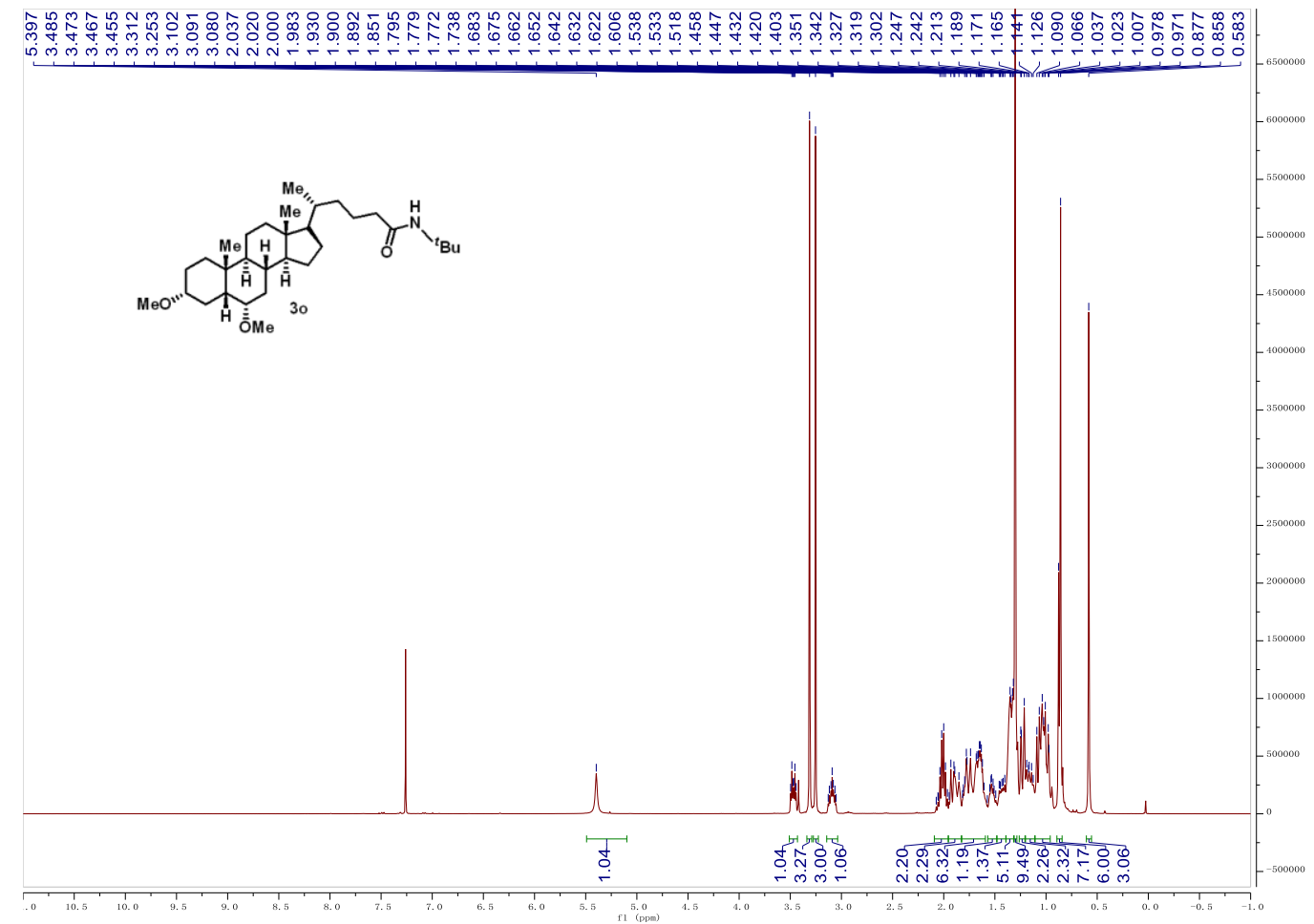

${ }^{13} \mathrm{C}$ NMR-spectrum(100 MHz, $\left.\mathrm{CDCl}_{3}\right)$ of 30

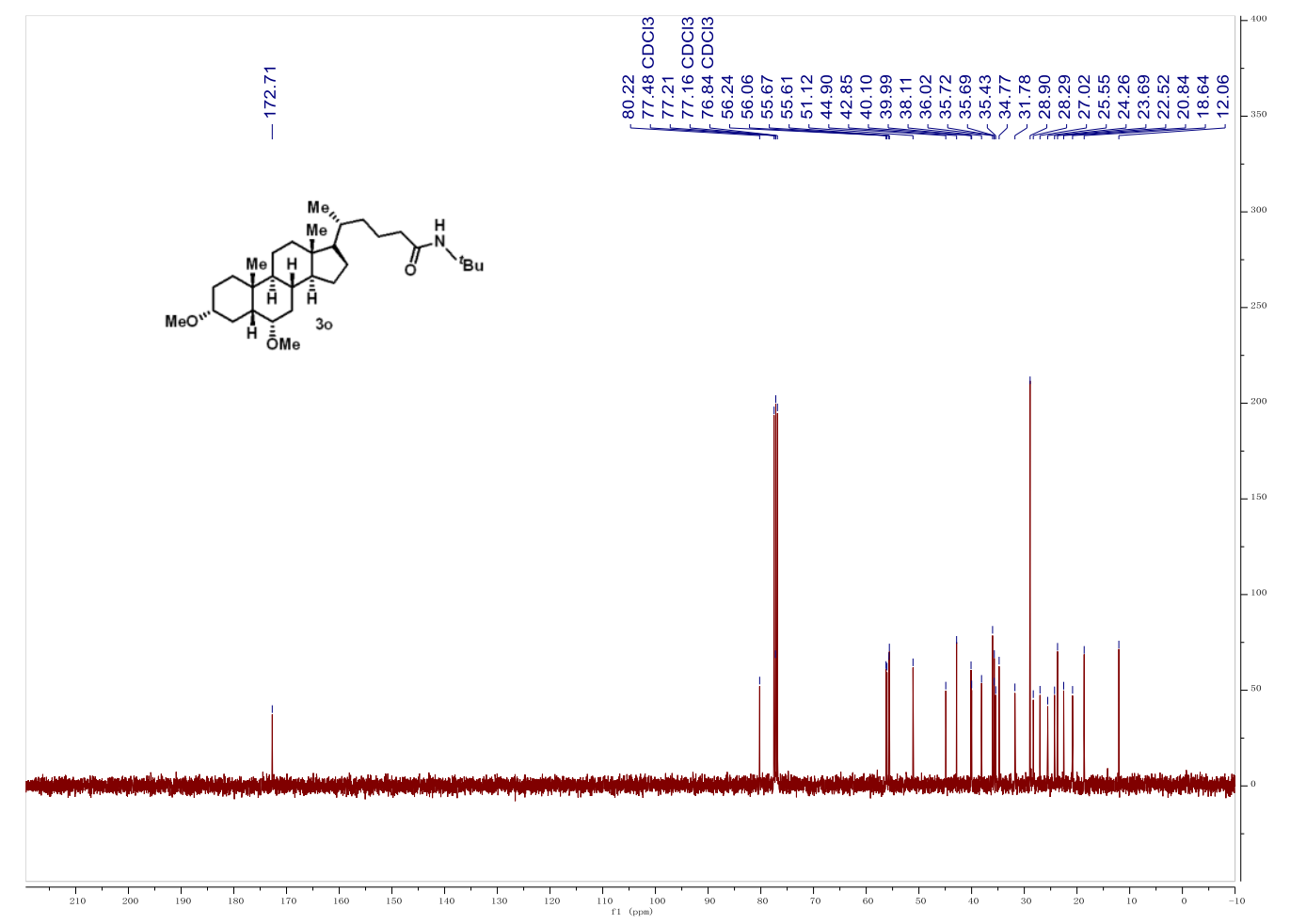


${ }^{1} \mathrm{H}$ NMR-spectrum(400 $\left.\mathrm{MHz}, \mathrm{CDCl}_{3}\right)$ of 3p

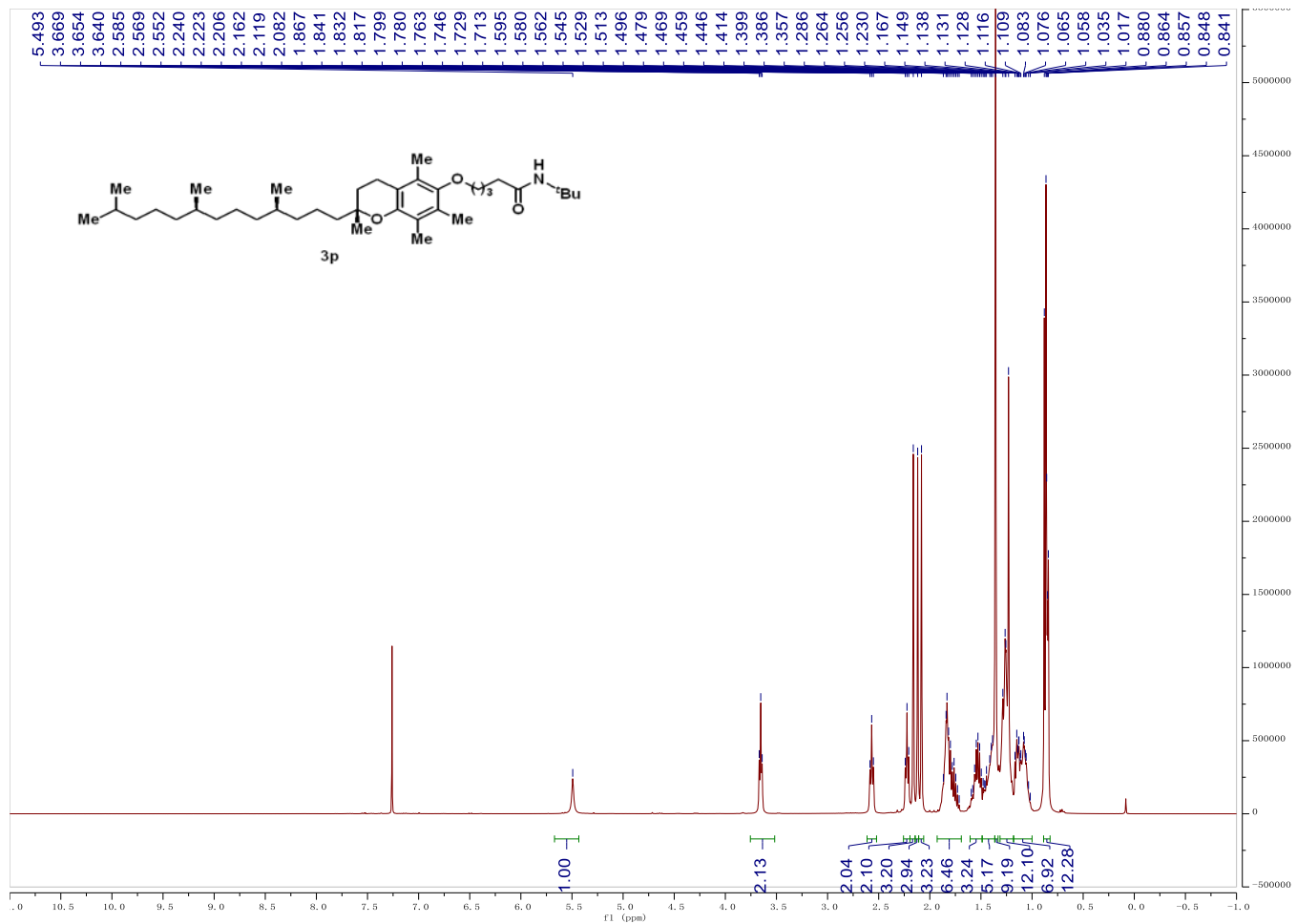

${ }^{13} \mathrm{C}$ NMR-spectrum(100 MHz, $\left.\mathrm{CDCl}_{3}\right)$ of 3p

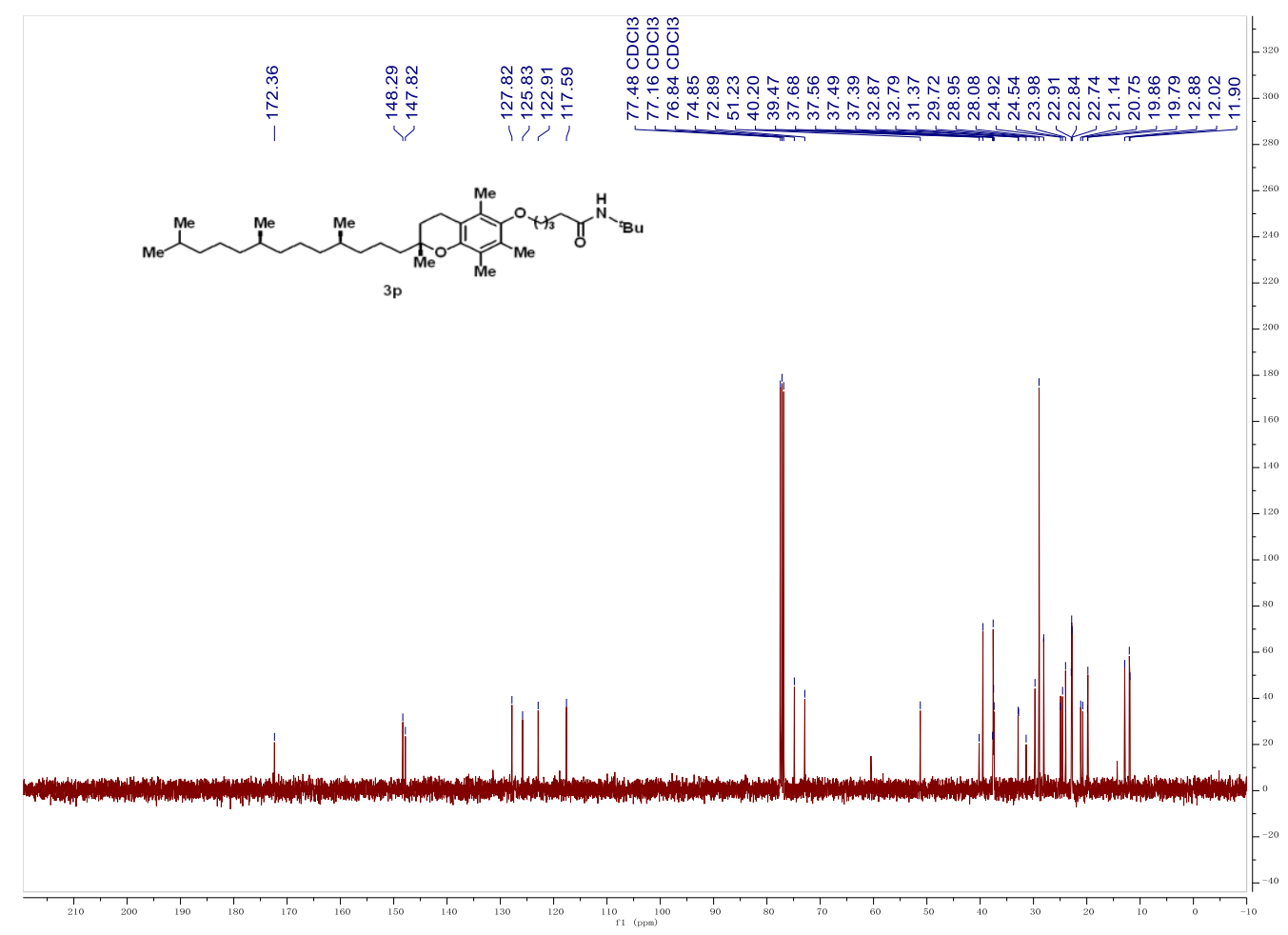


${ }^{1} \mathrm{H}$ NMR-spectrum(400 $\left.\mathrm{MHz}, \mathrm{CDCl}_{3}\right)$ of $3 q$

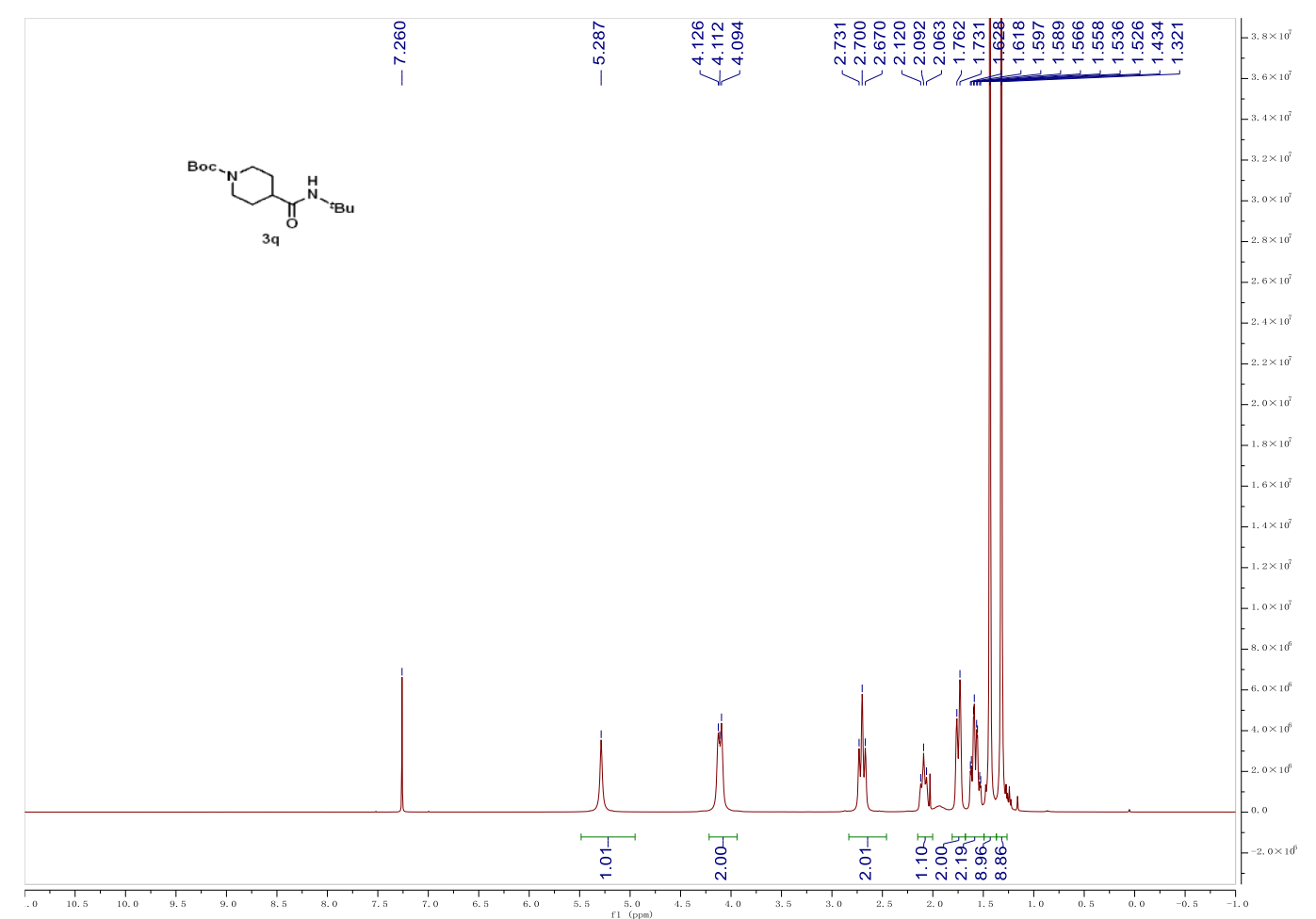

${ }^{13} \mathrm{C}$ NMR-spectrum(100 MHz, $\left.\mathrm{CDCl}_{3}\right)$ of $3 q$

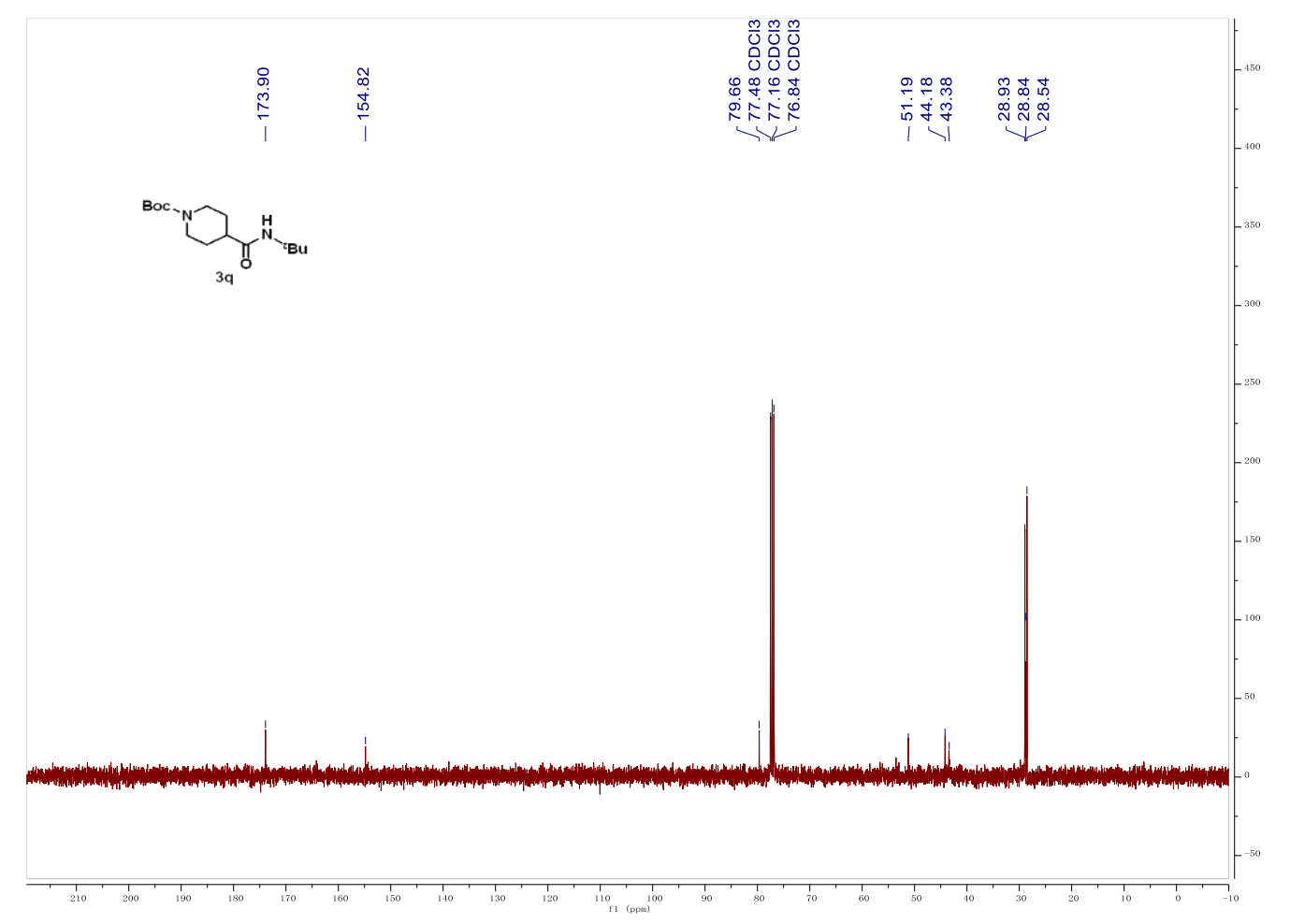


${ }^{1} \mathrm{H}$ NMR-spectrum(400 $\left.\mathrm{MHz}, \mathrm{CDCl}_{3}\right)$ of $3 \mathrm{r}$

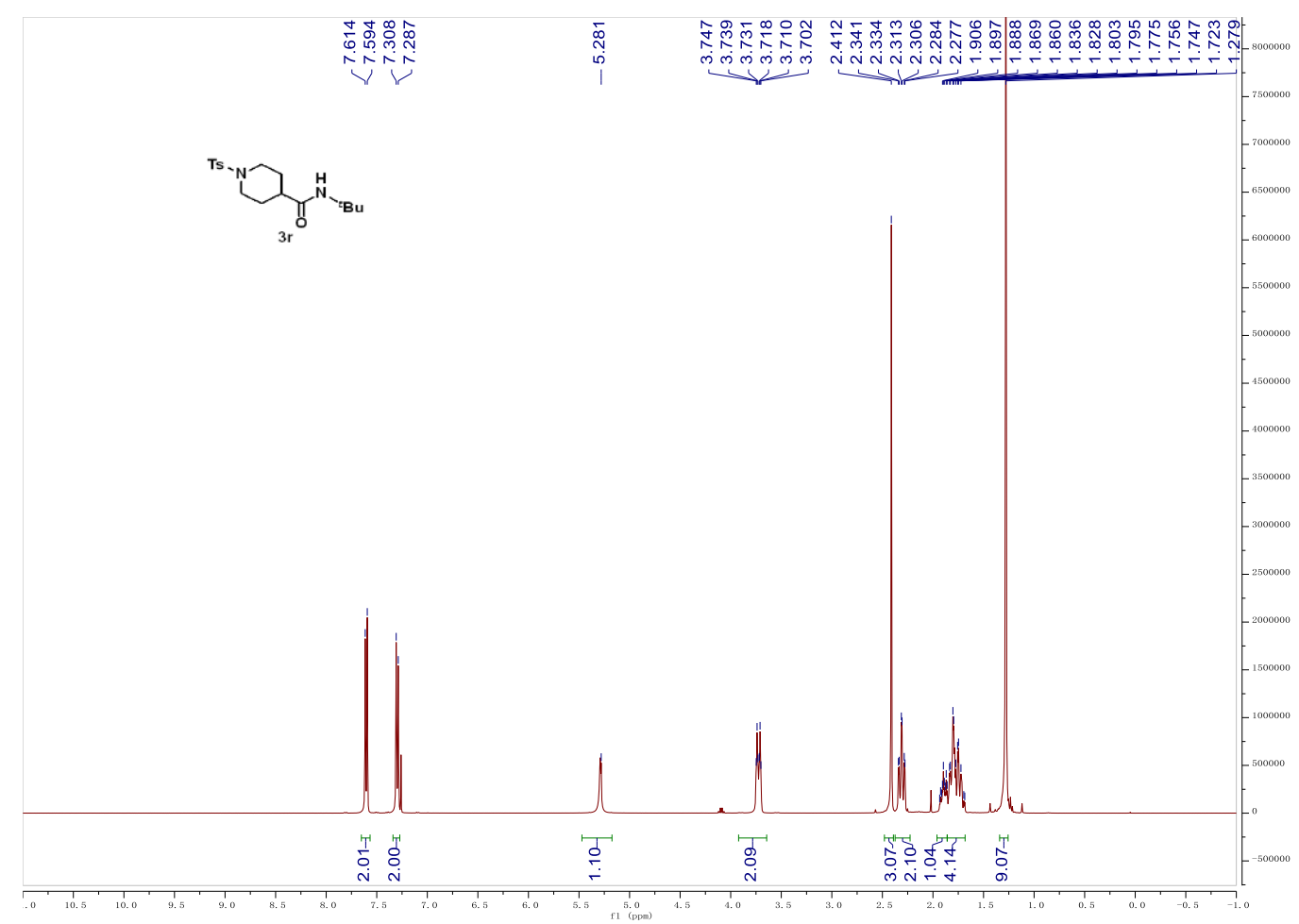

${ }^{13} \mathrm{C}$ NMR-spectrum(100 MHz, $\left.\mathrm{CDCl}_{3}\right)$ of $3 \mathrm{r}$

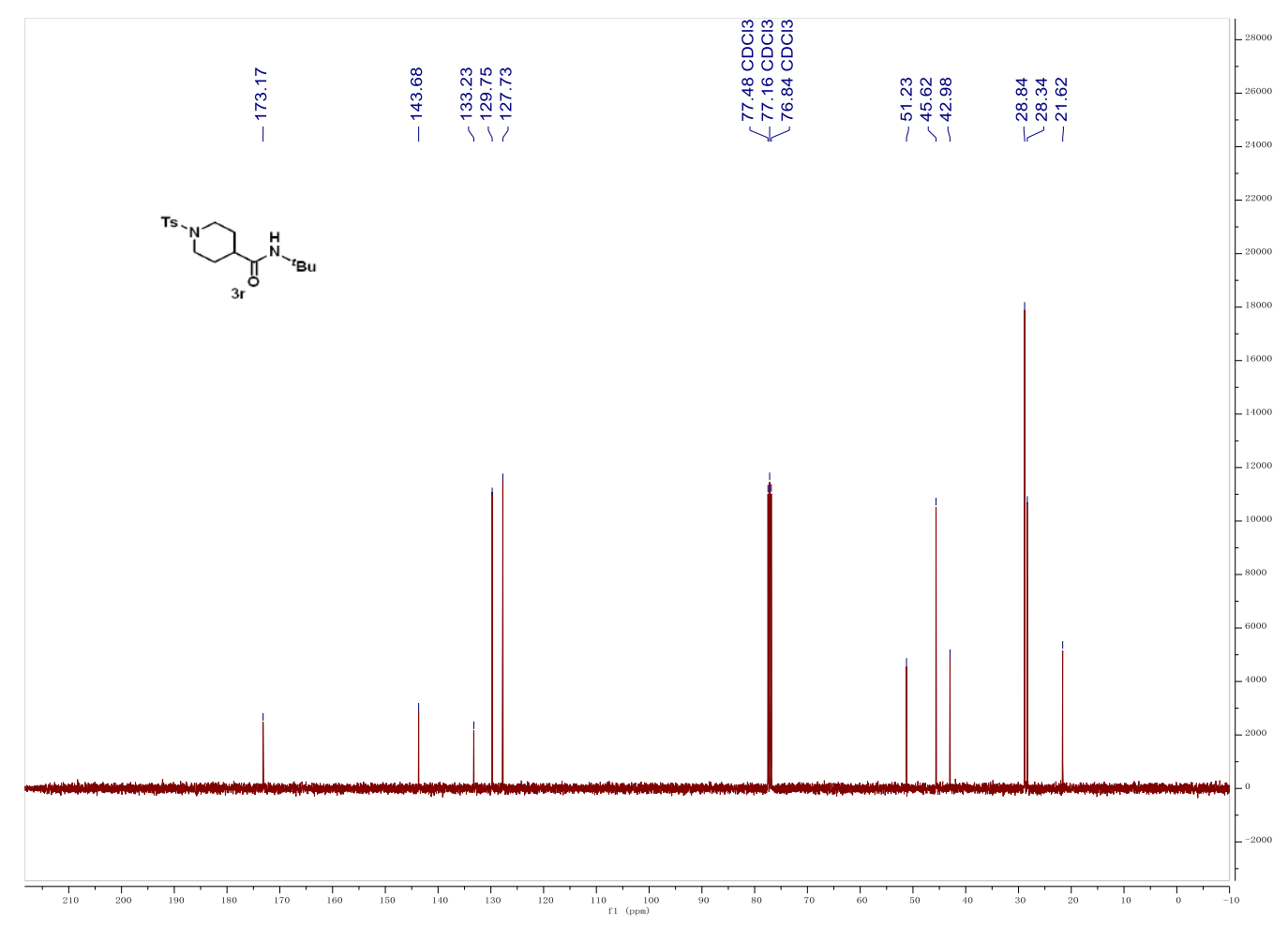


${ }^{1} \mathrm{H}$ NMR-spectrum(400 $\left.\mathrm{MHz}, \mathrm{CDCl}_{3}\right)$ of $3 \mathrm{~s}$

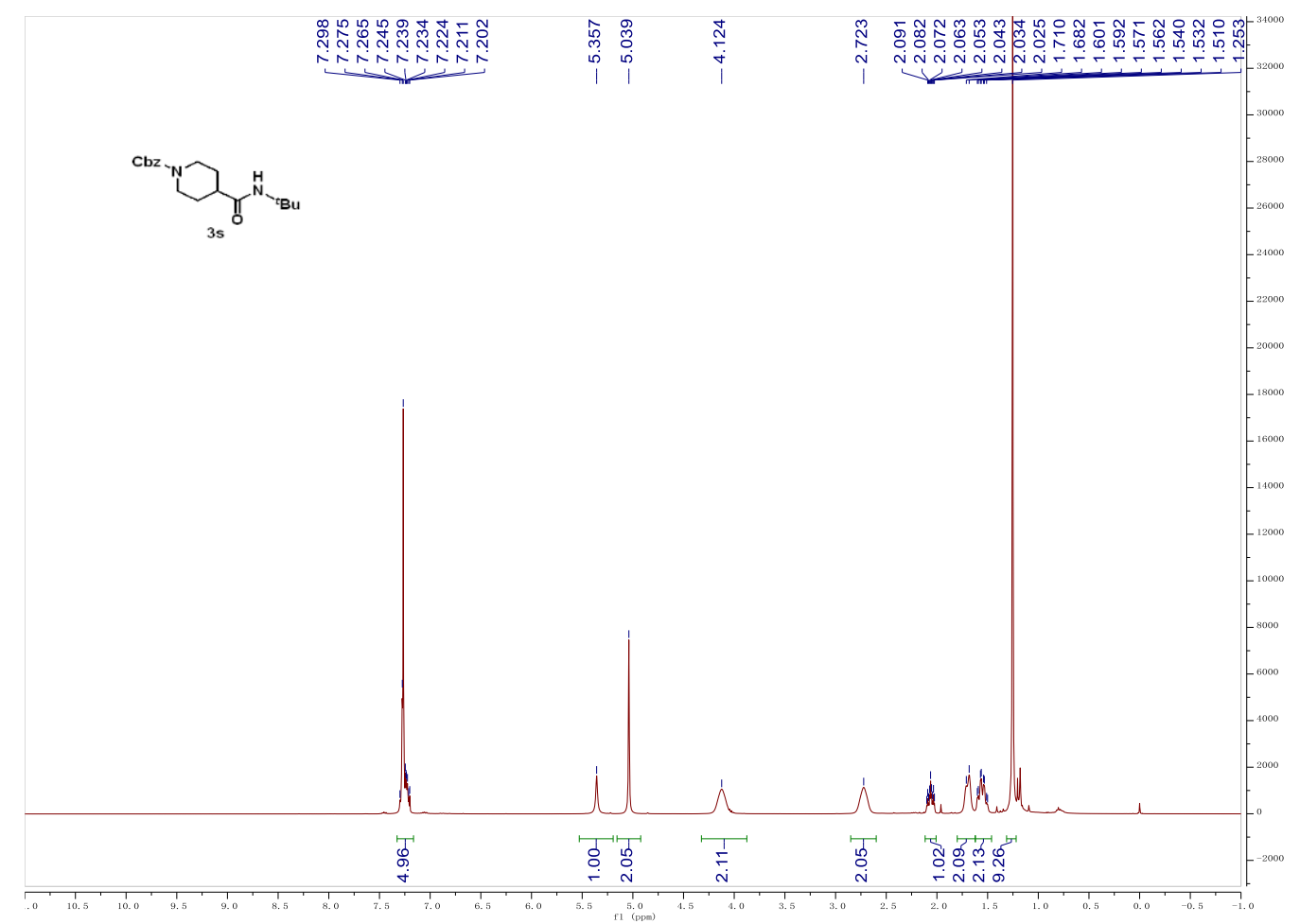

${ }^{13} \mathrm{C}$ NMR-spectrum(100 MHz, $\left.\mathrm{CDCl}_{3}\right)$ of $3 \mathrm{~s}$

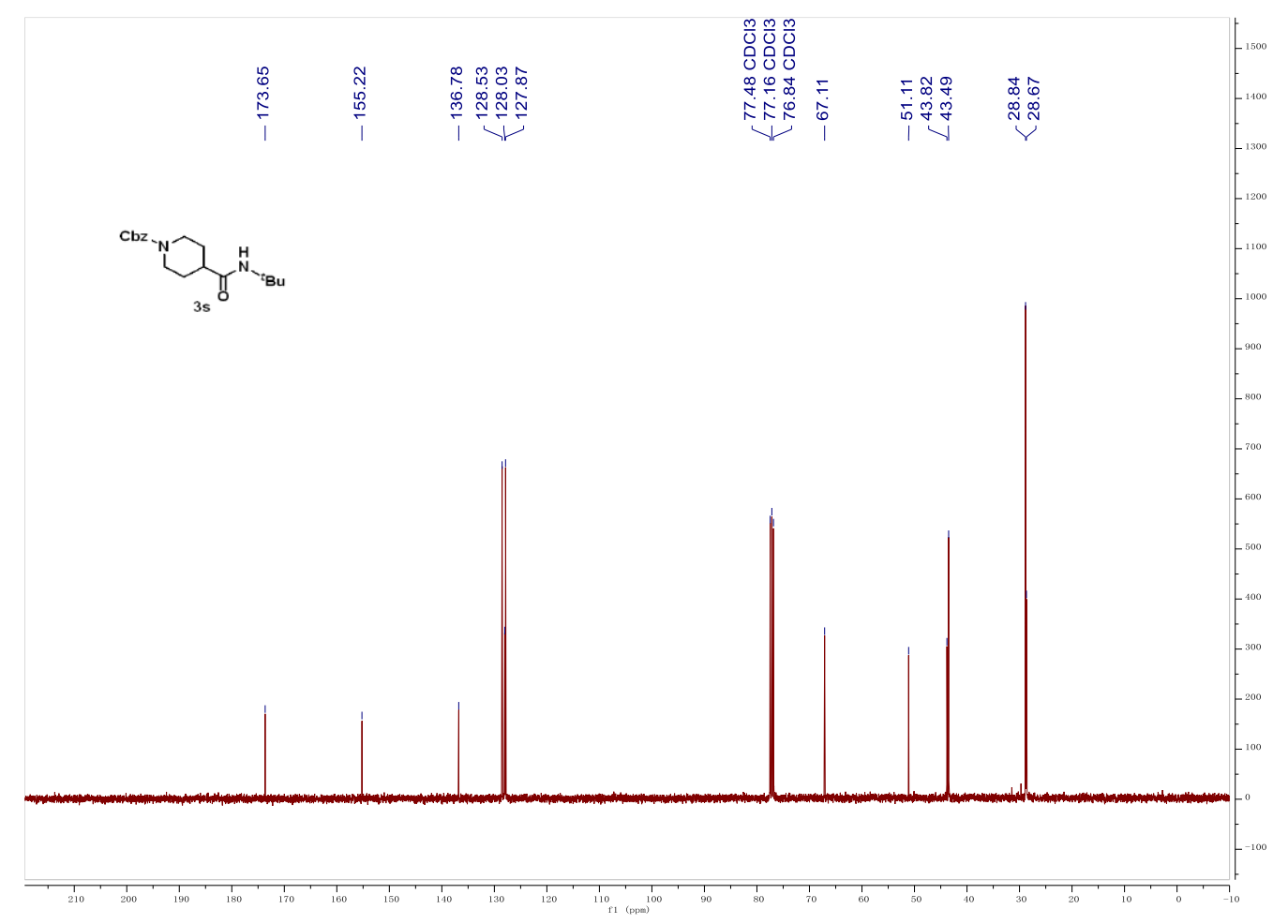


${ }^{1} \mathrm{H}$ NMR-spectrum(400 $\left.\mathrm{MHz}, \mathrm{CDCl}_{3}\right)$ of $3 \mathrm{t}$

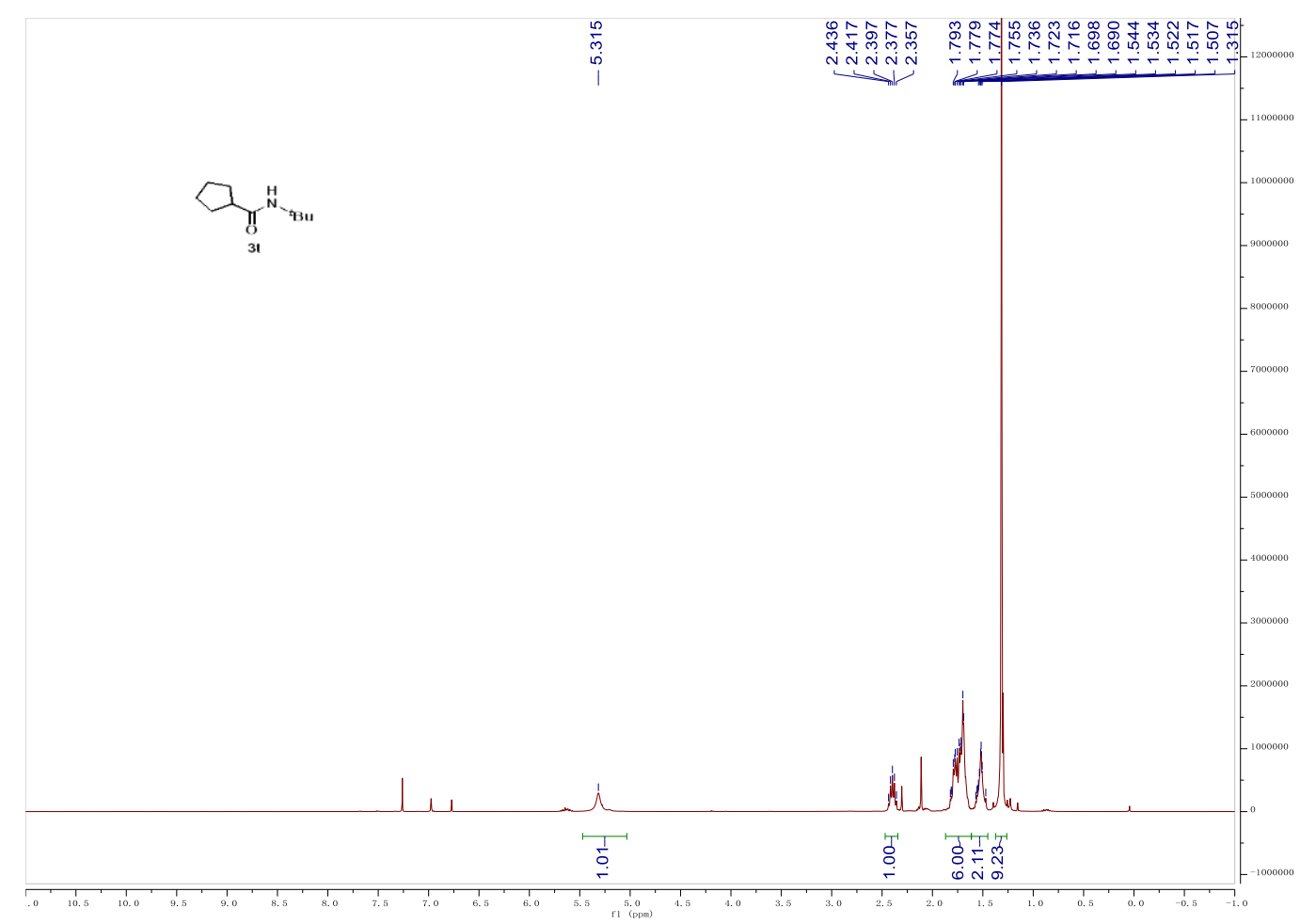

${ }^{13} \mathrm{C}$ NMR-spectrum(100 MHz, $\left.\mathrm{CDCl}_{3}\right)$ of $3 \mathrm{t}$

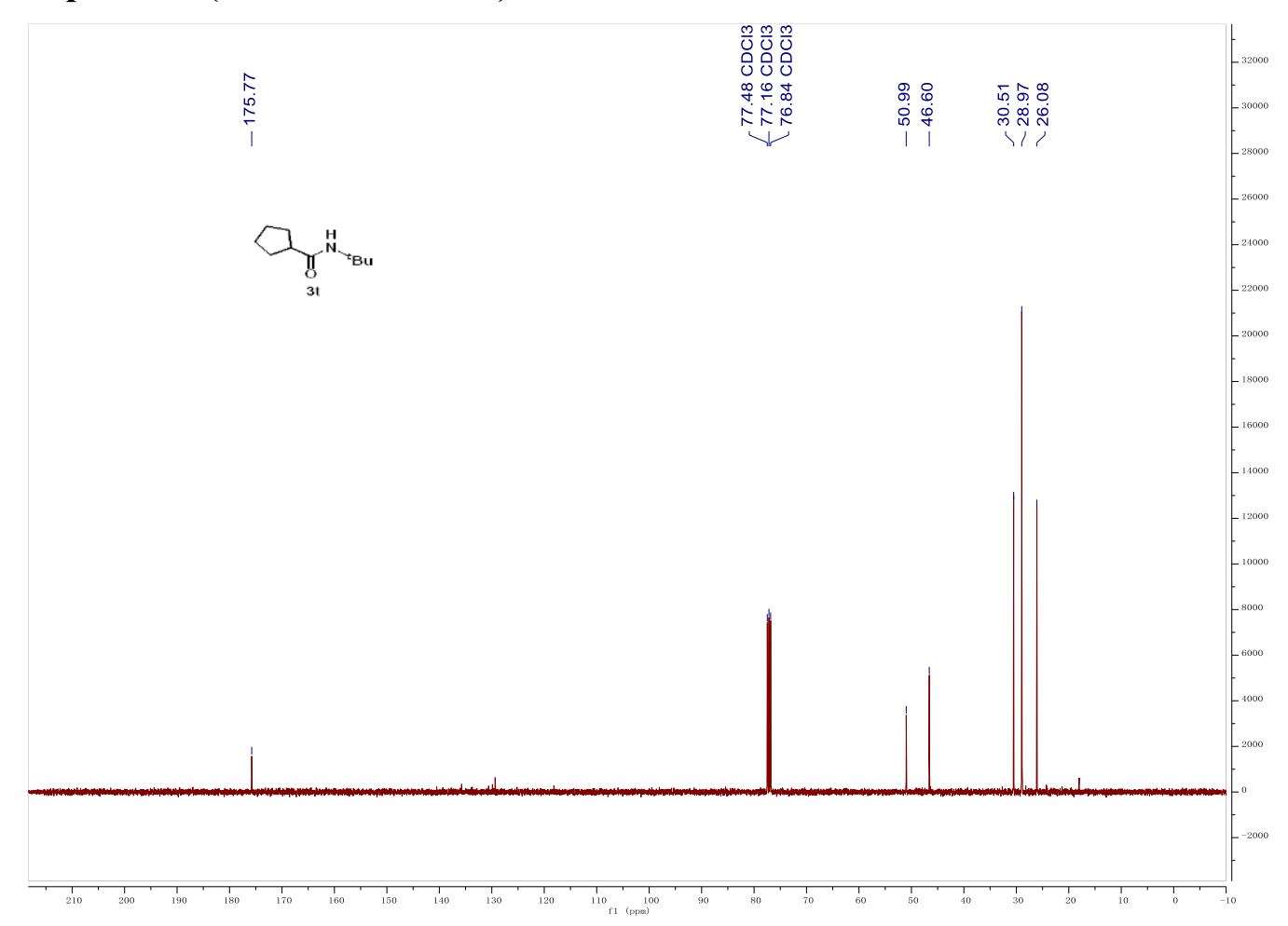


${ }^{1} \mathrm{H}$ NMR-spectrum(400 $\left.\mathrm{MHz}, \mathrm{CDCl}_{3}\right)$ of $3 \mathrm{u}$

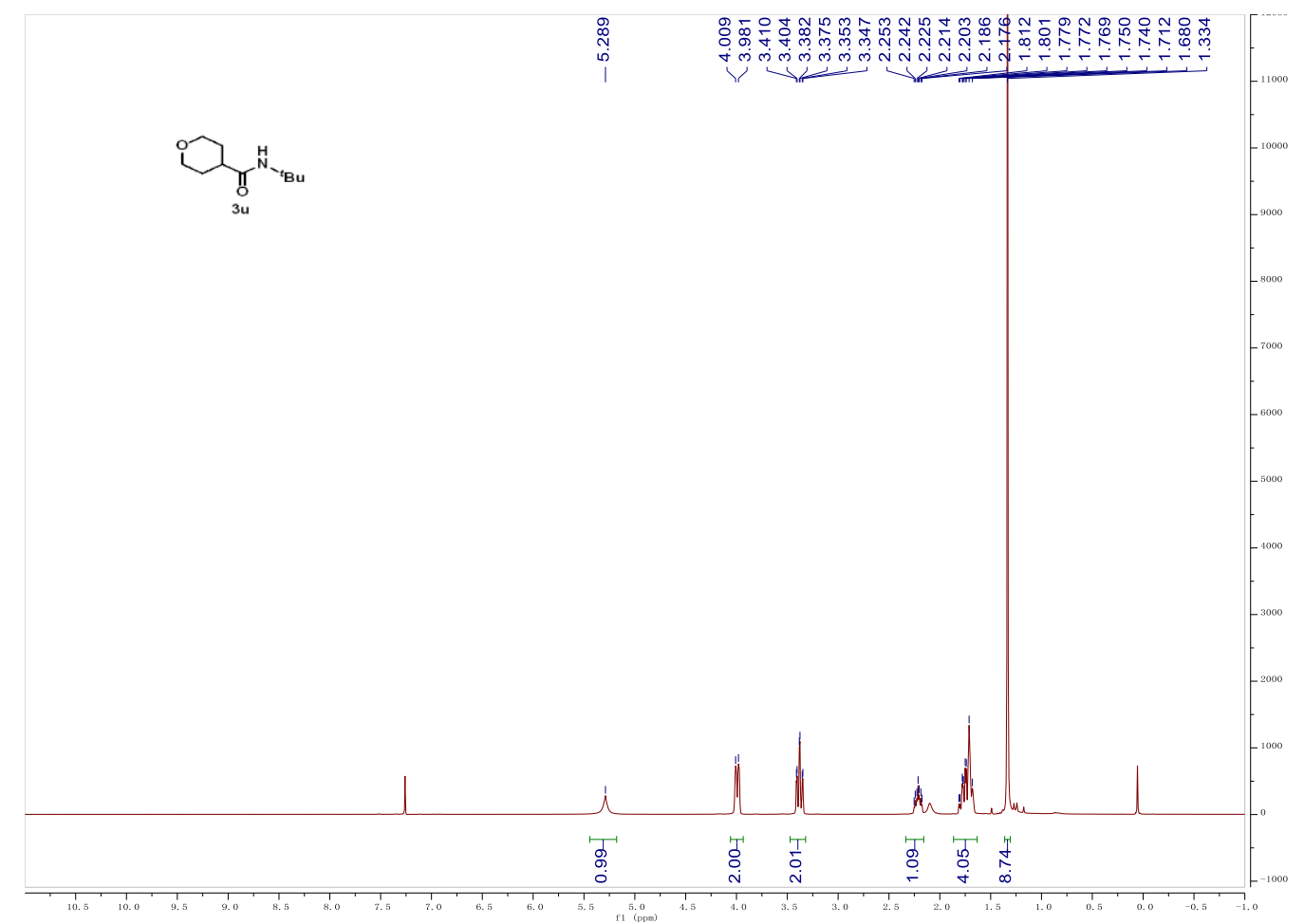

${ }^{13} \mathrm{C}$ NMR-spectrum(100 MHz, $\left.\mathrm{CDCl}_{3}\right)$ of $3 \mathrm{u}$

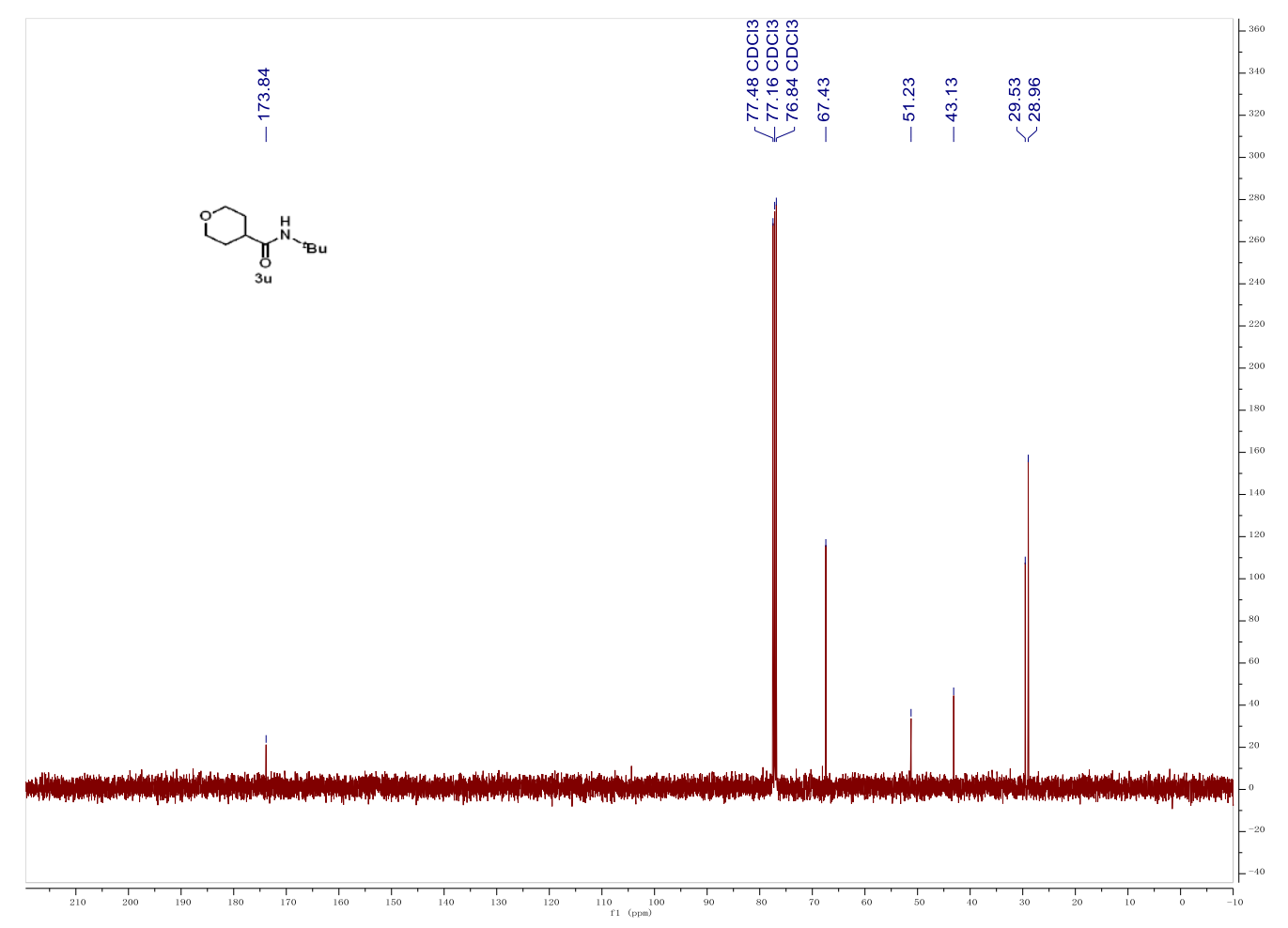


${ }^{1} \mathrm{H}$ NMR-spectrum(400 $\left.\mathrm{MHz}, \mathrm{CDCl}_{3}\right)$ of $3 \mathrm{v}$

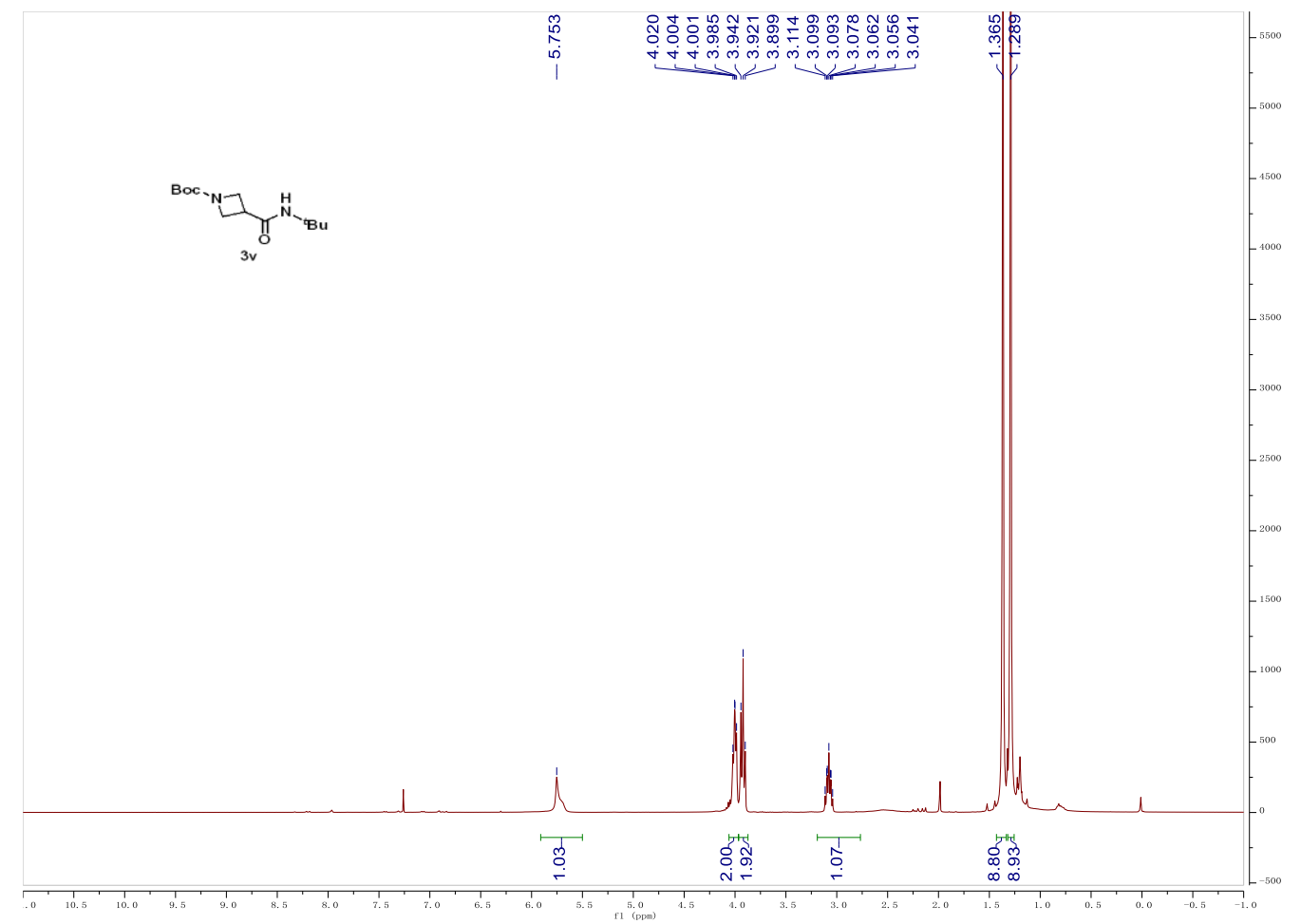

${ }^{13} \mathrm{C}$ NMR-spectrum(100 MHz, $\left.\mathrm{CDCl}_{3}\right)$ of $3 \mathrm{v}$

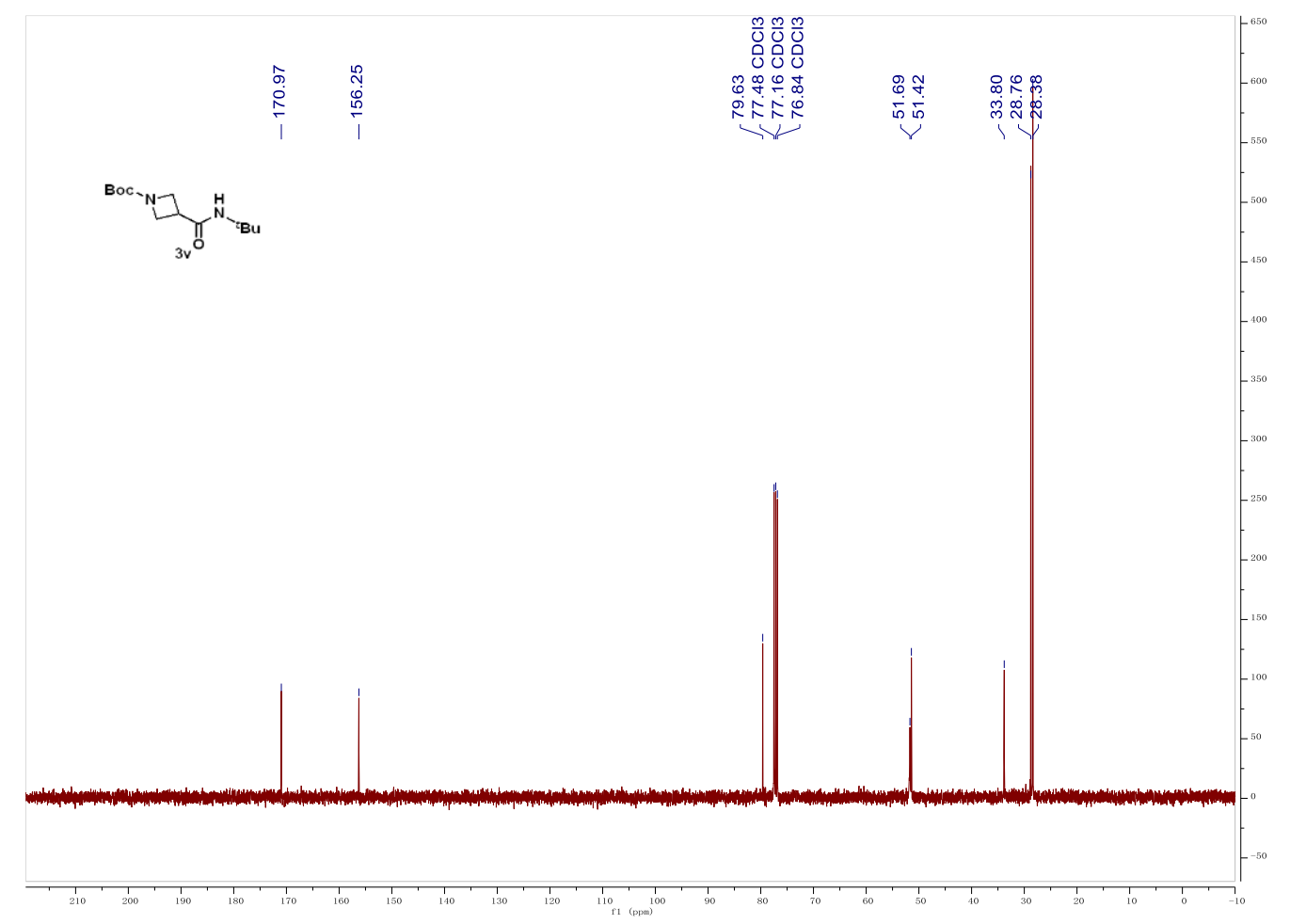


${ }^{1} \mathrm{H}$ NMR-spectrum(400 $\left.\mathrm{MHz}, \mathrm{CDCl}_{3}\right)$ of $3 \mathrm{w}$

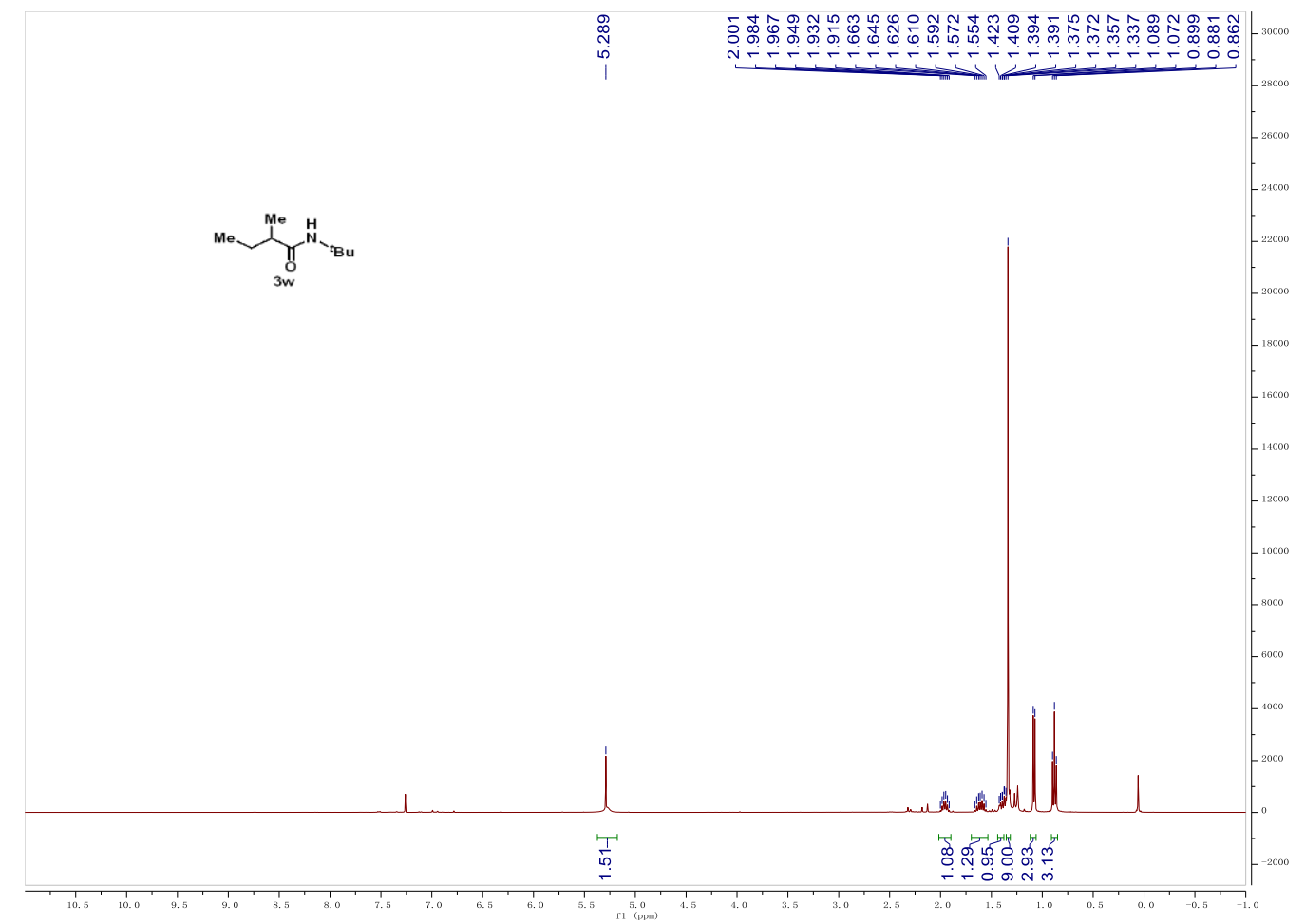

${ }^{13} \mathrm{C}$ NMR-spectrum(100 $\left.\mathrm{MHz}, \mathrm{CDCl}_{3}\right)$ of $3 \mathrm{w}$

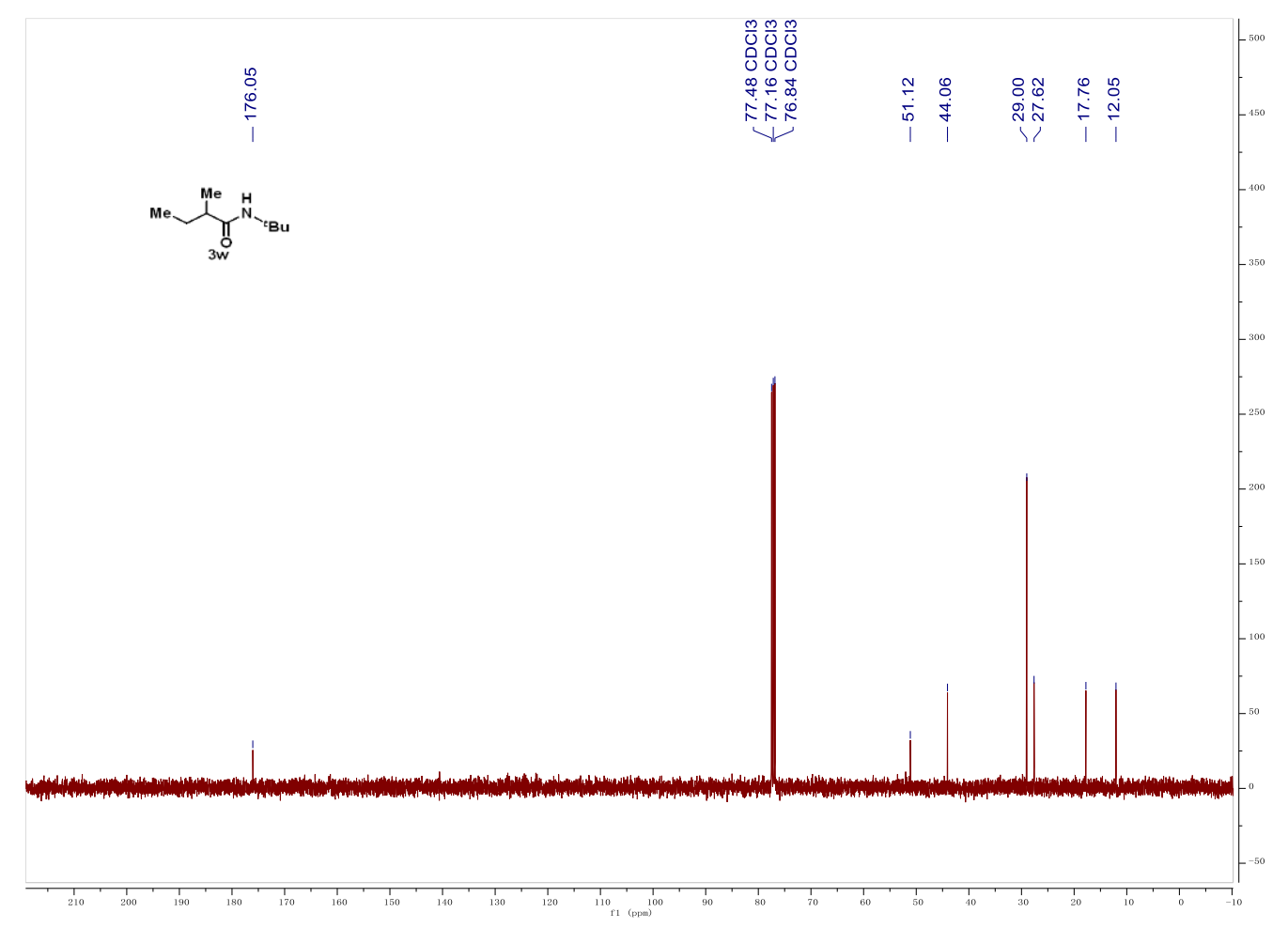


${ }^{1} \mathrm{H}$ NMR-spectrum(400 $\left.\mathrm{MHz}, \mathrm{CDCl}_{3}\right)$ of $3 \mathrm{x}$

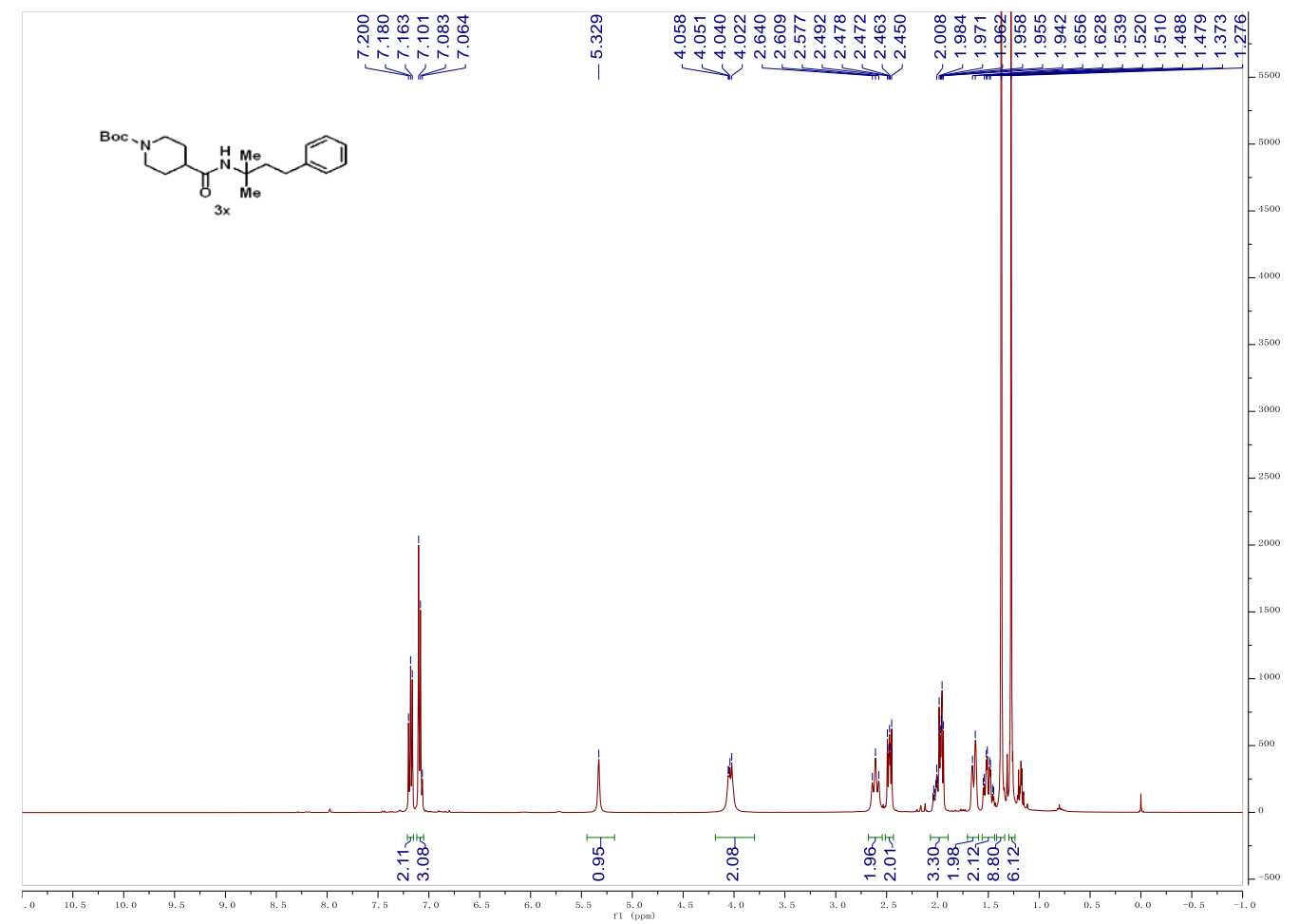

${ }^{13} \mathrm{C}$ NMR-spectrum(100 MHz, $\left.\mathrm{CDCl}_{3}\right)$ of $3 x$

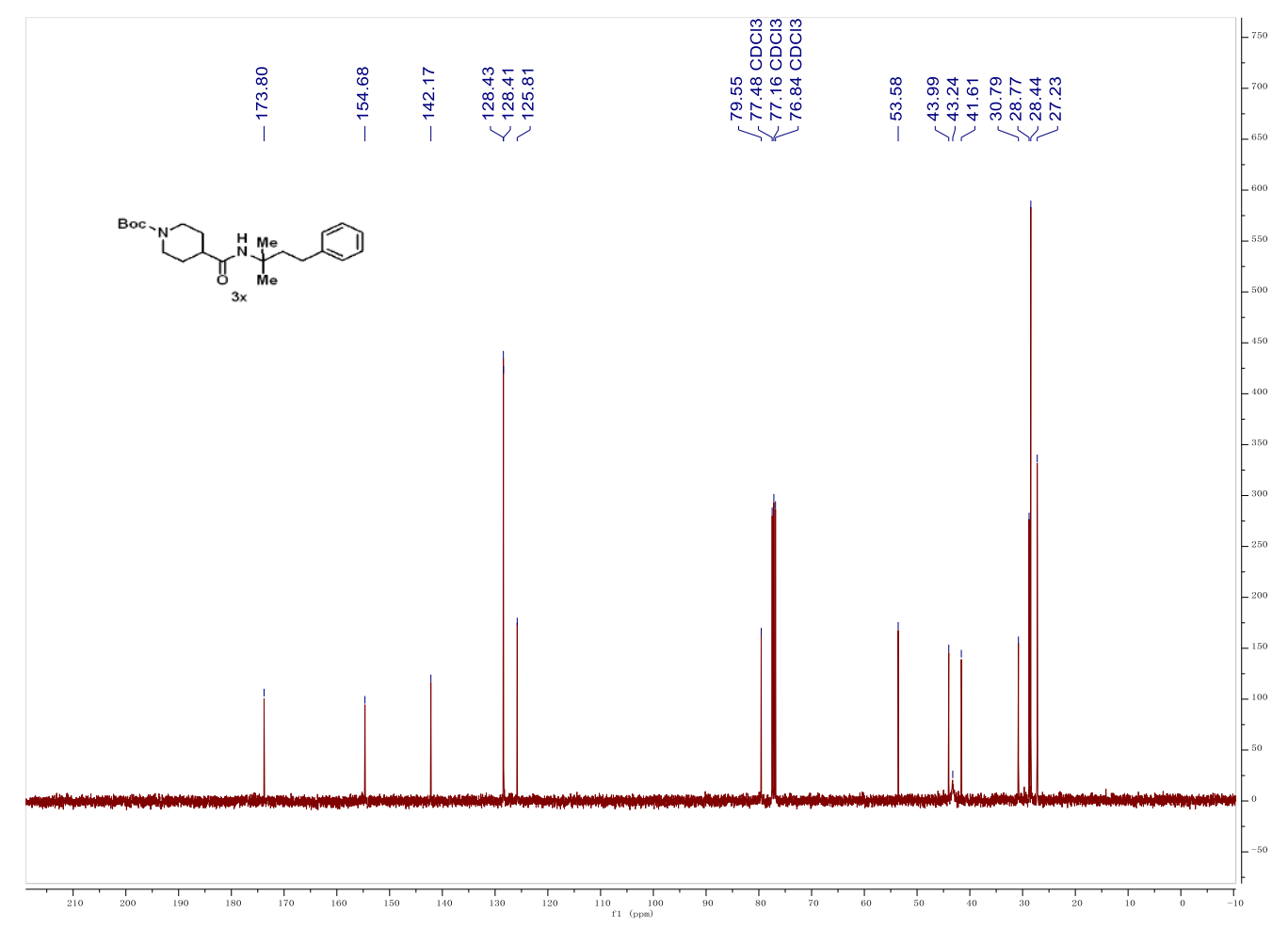


${ }^{1} \mathrm{H}$ NMR-spectrum(400 $\left.\mathrm{MHz}, \mathrm{CDCl}_{3}\right)$ of $3 \mathrm{y}$

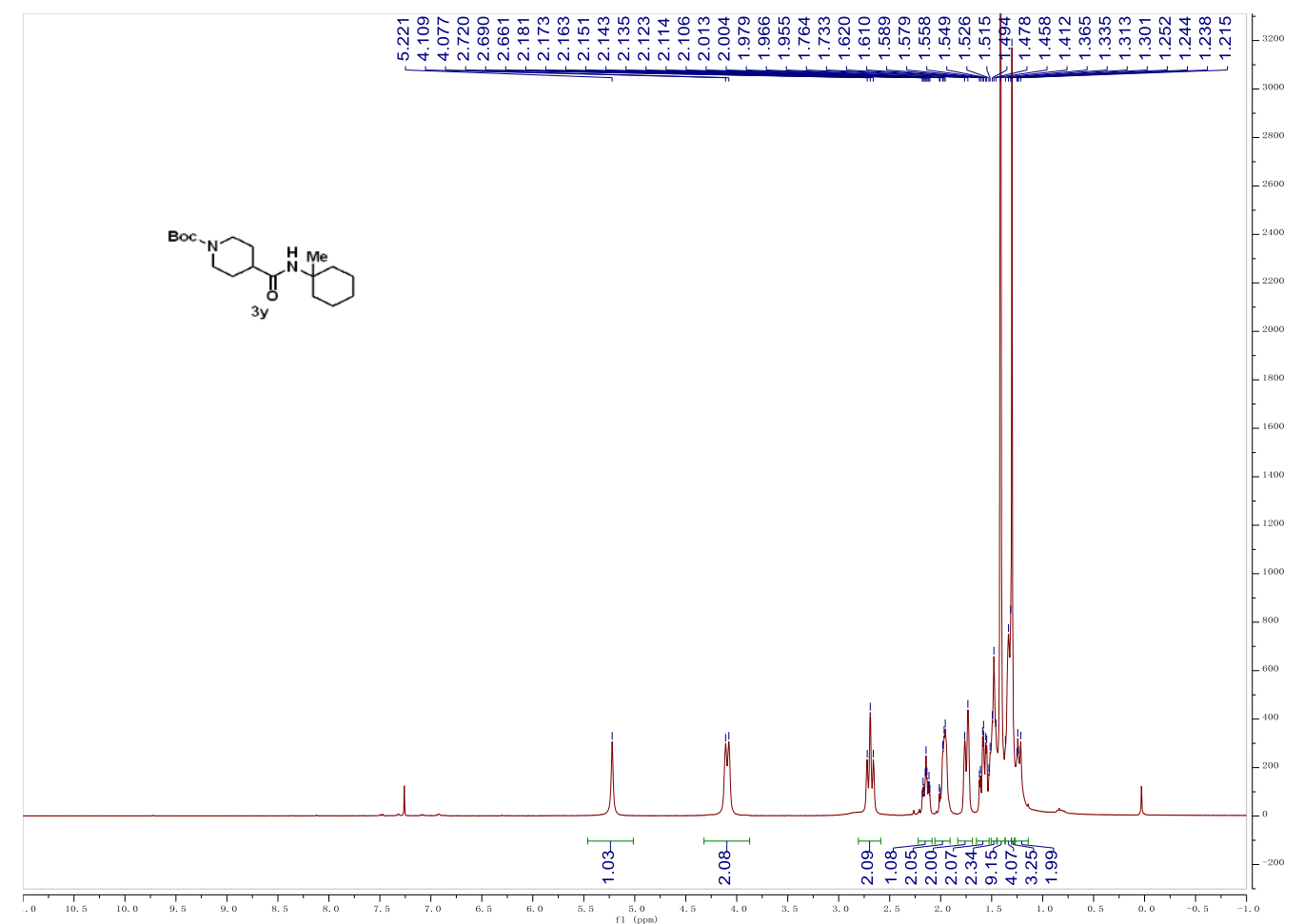

${ }^{13} \mathrm{C}$ NMR-spectrum(100 MHz, $\left.\mathrm{CDCl}_{3}\right)$ of $3 \mathrm{y}$

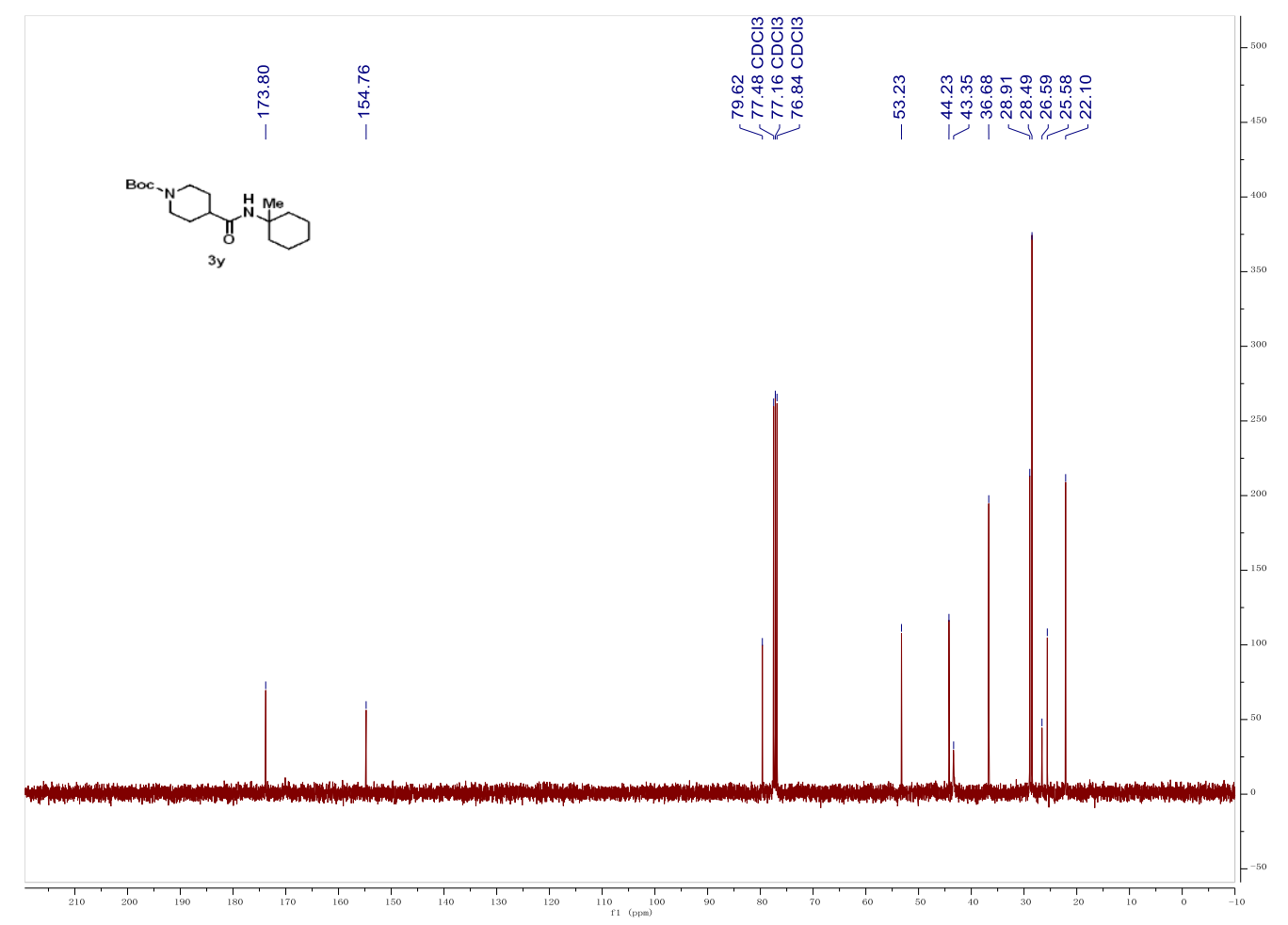


${ }^{1} \mathrm{H}$ NMR-spectrum(400 $\left.\mathrm{MHz}, \mathrm{CDCl}_{3}\right)$ of $3 \mathrm{z}$

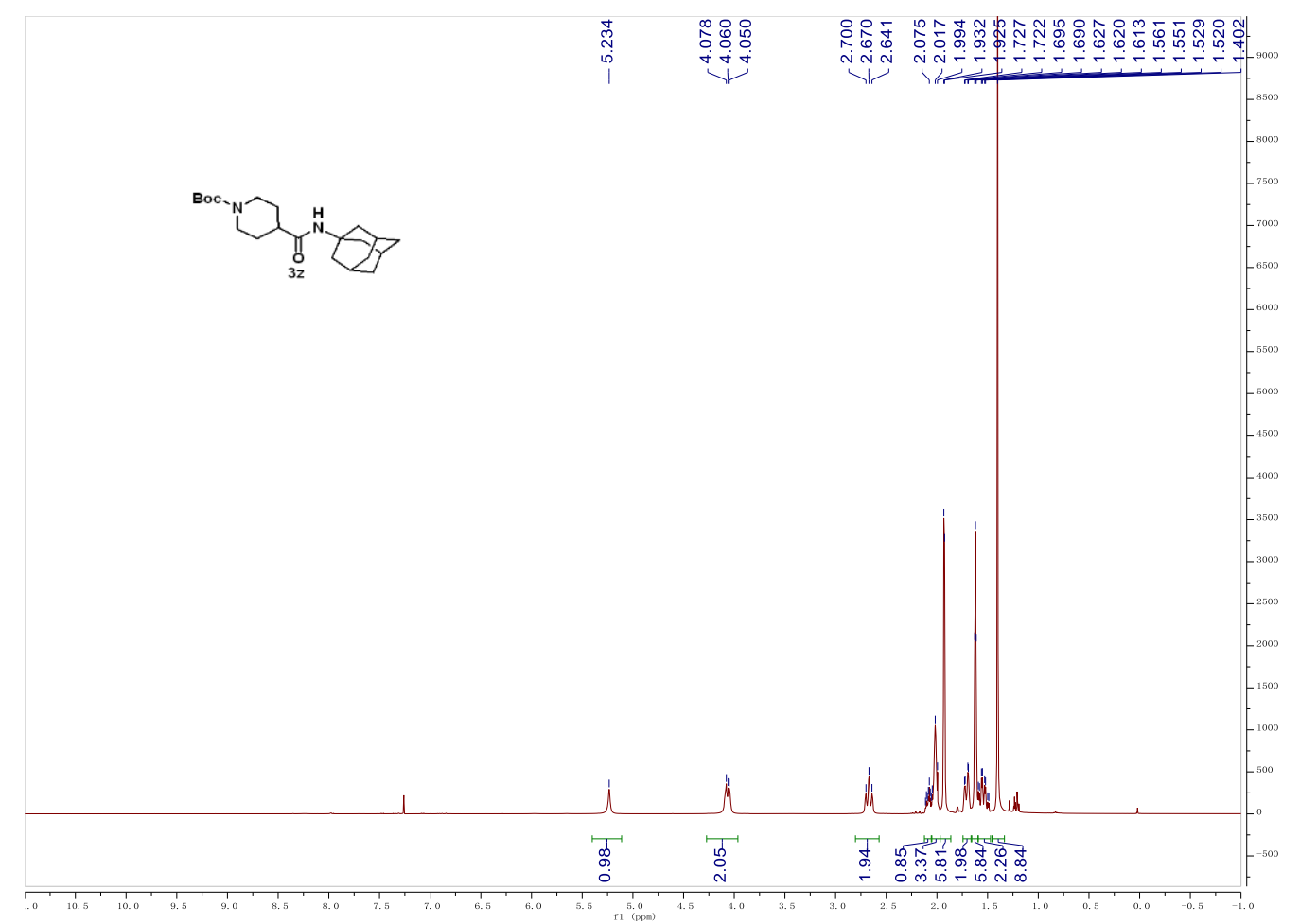

${ }^{13} \mathrm{C}$ NMR-spectrum(100 MHz, $\left.\mathrm{CDCl}_{3}\right)$ of $3 \mathrm{z}$

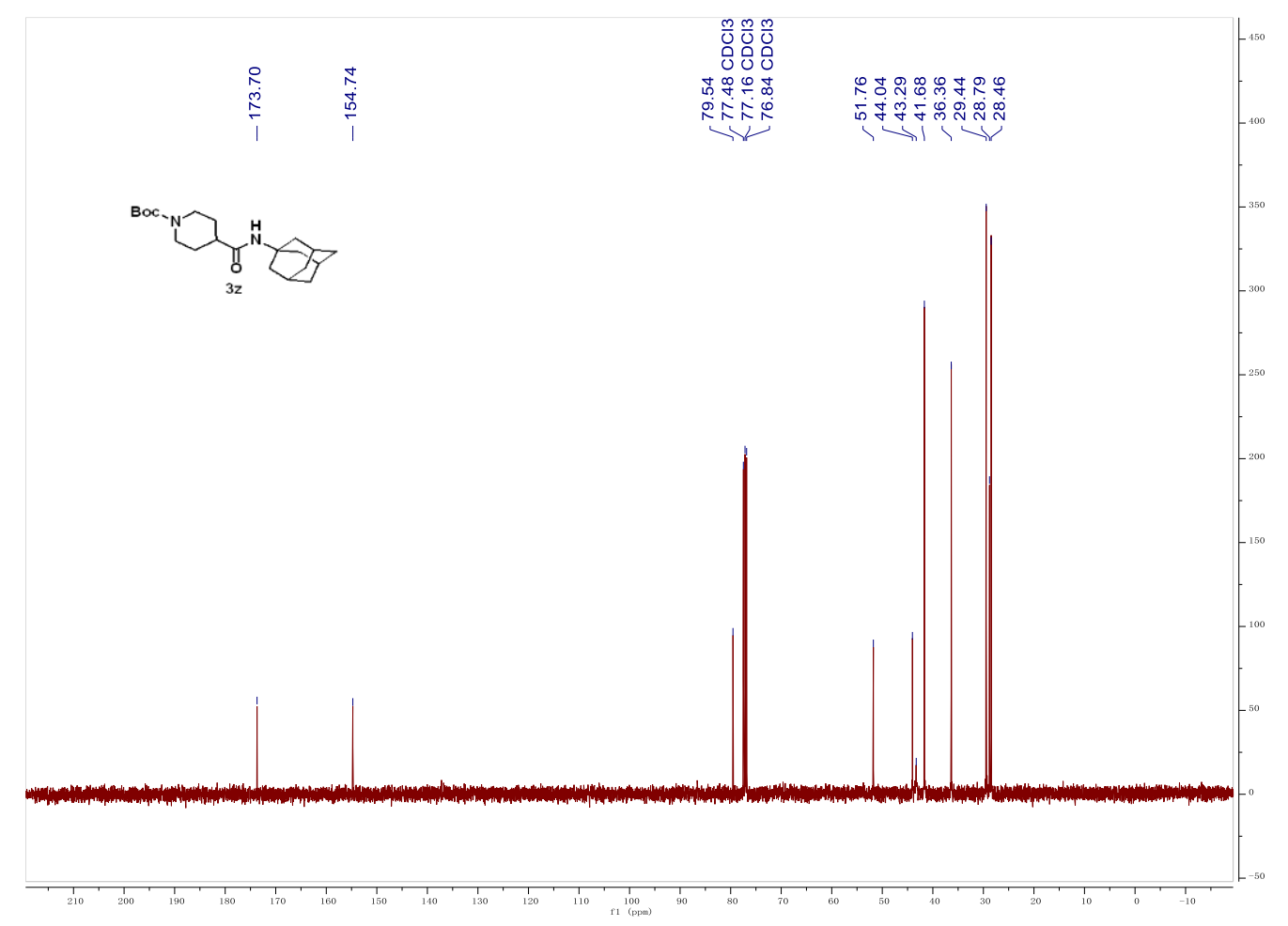


${ }^{1} \mathrm{H}$ NMR-spectrum(400 $\left.\mathrm{MHz}, \mathrm{CDCl}_{3}\right)$ of $3 \mathrm{aa}$

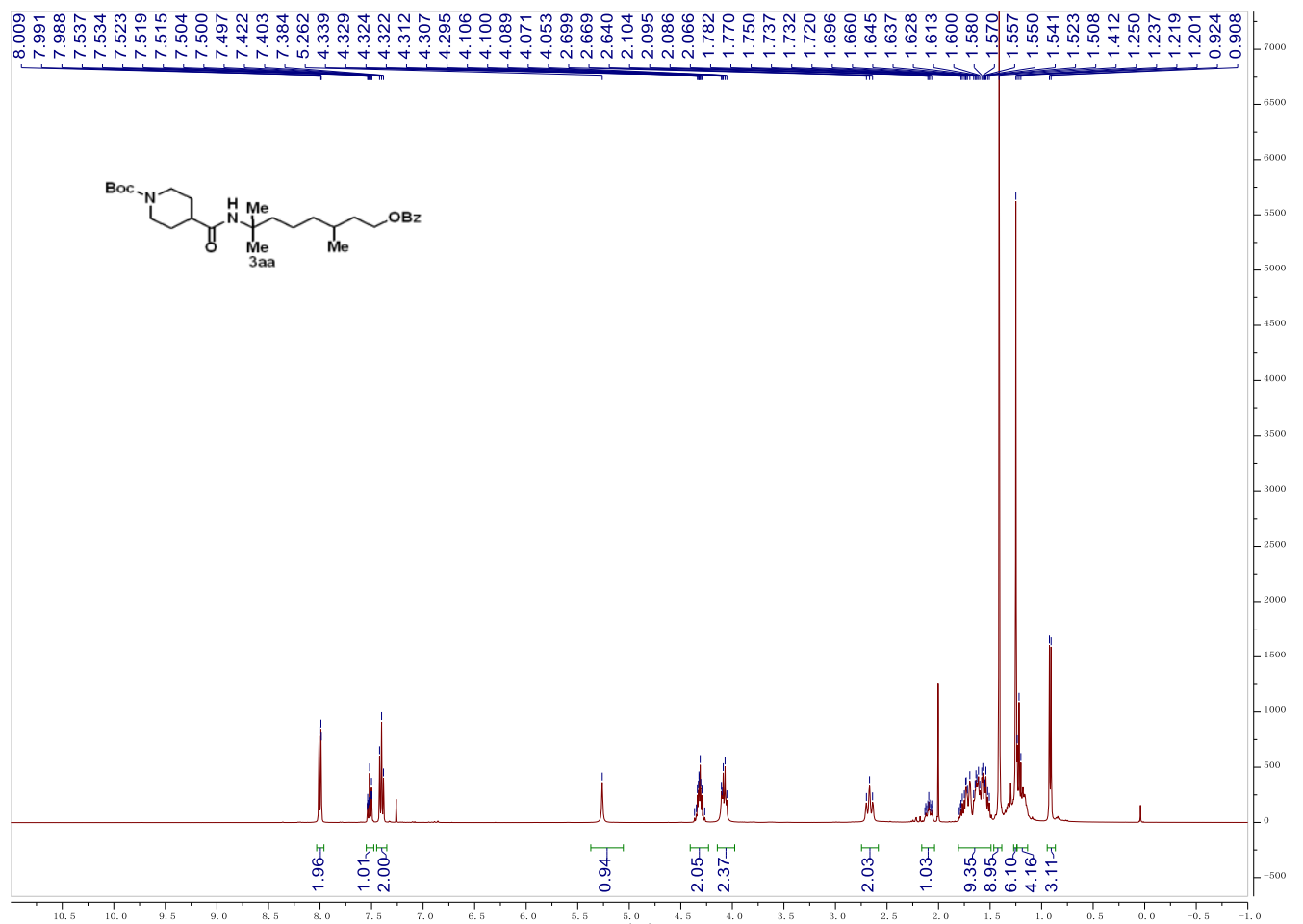

${ }^{13} \mathrm{C}$ NMR-spectrum(100 MHz, $\left.\mathrm{CDCl}_{3}\right)$ of $3 \mathrm{aa}$

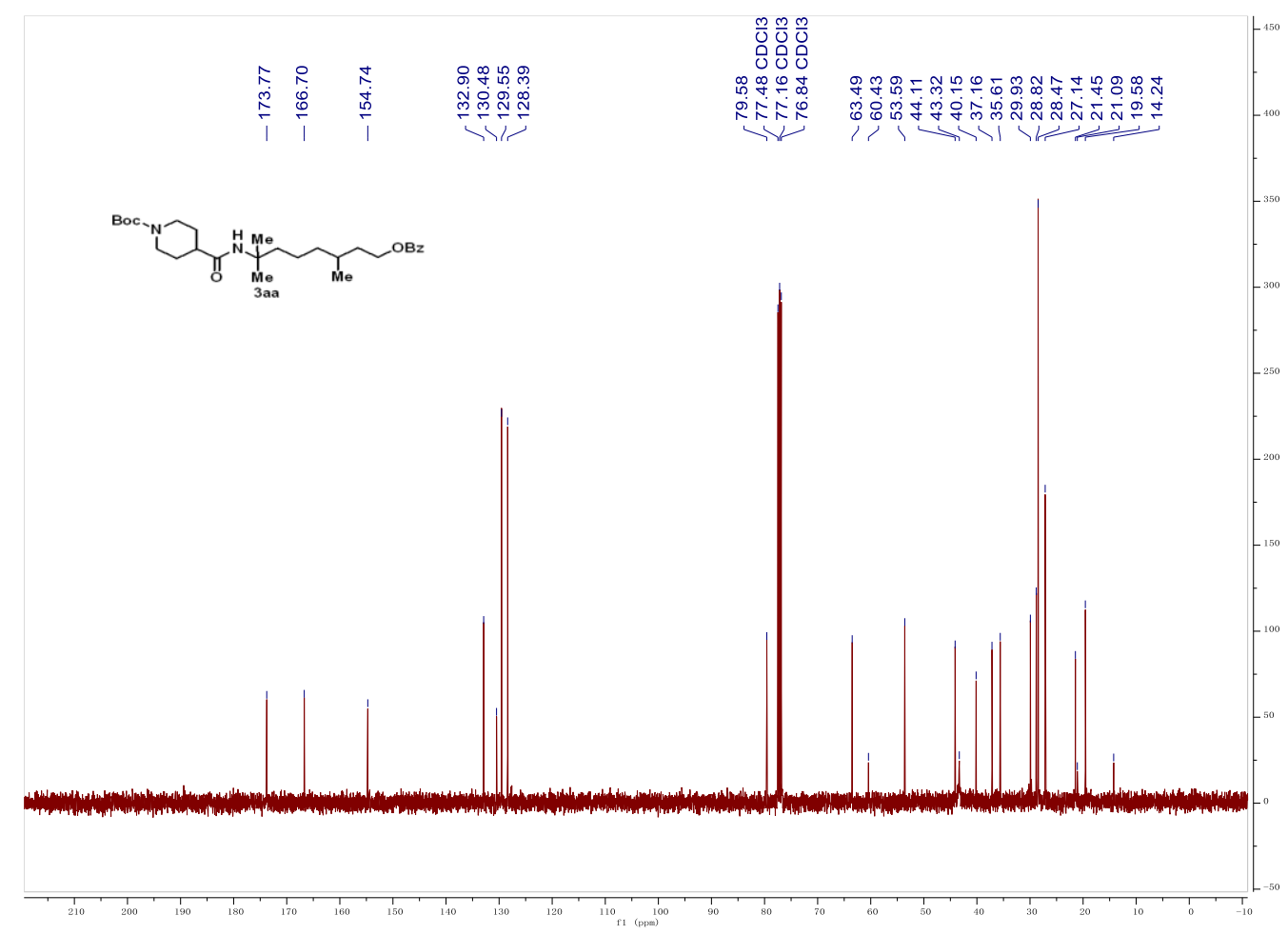


${ }^{1} \mathrm{H}$ NMR-spectrum(400 $\left.\mathrm{MHz}, \mathrm{CDCl}_{3}\right)$ of 4

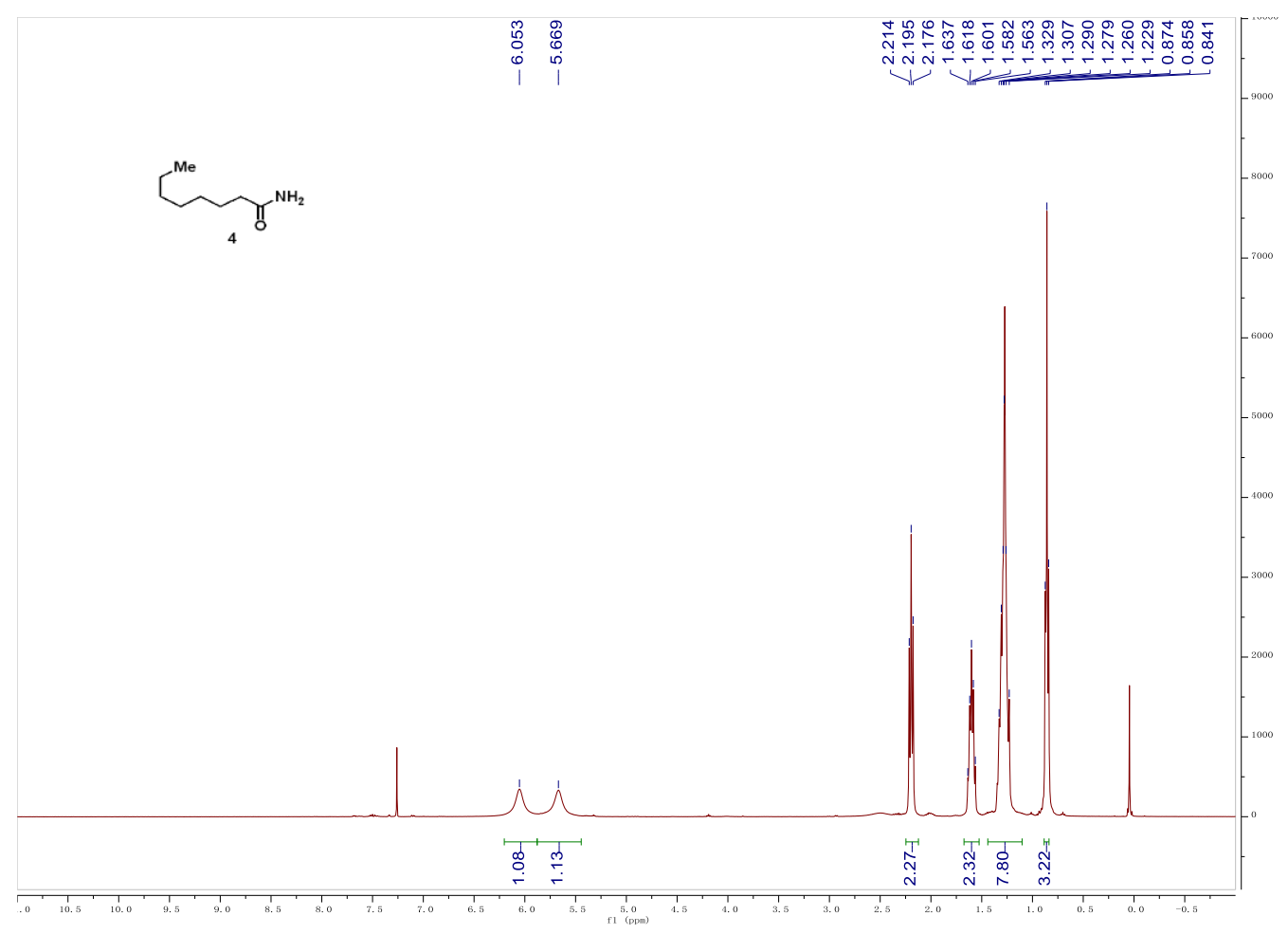

${ }^{1} \mathrm{H}$ NMR-spectrum(400 $\left.\mathrm{MHz}, \mathrm{CDCl}_{3}\right)$ of 5

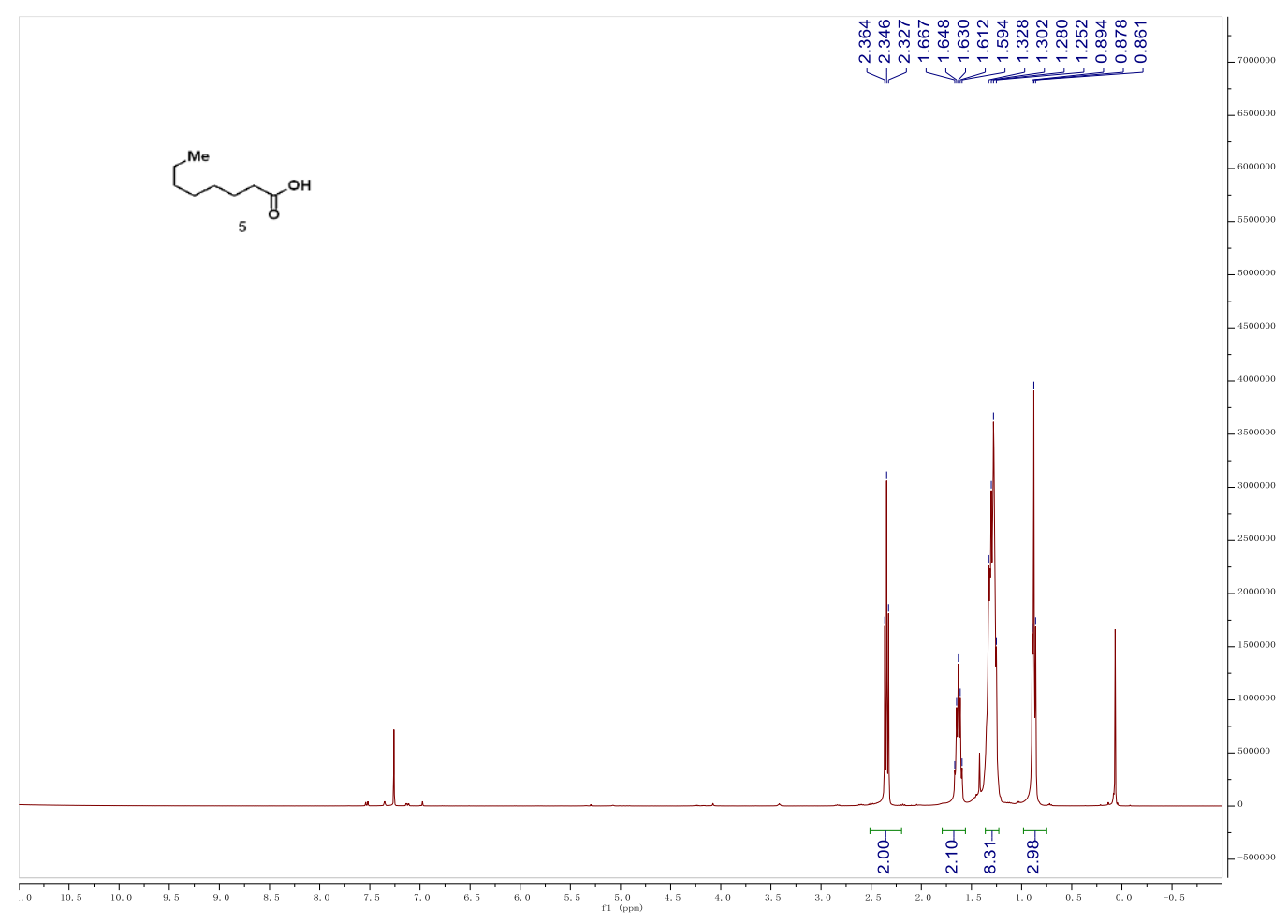

50 
${ }^{13} \mathrm{C}$ NMR-spectrum(100 $\left.\mathrm{MHz}, \mathrm{CDCl}_{3}\right)$ of 5

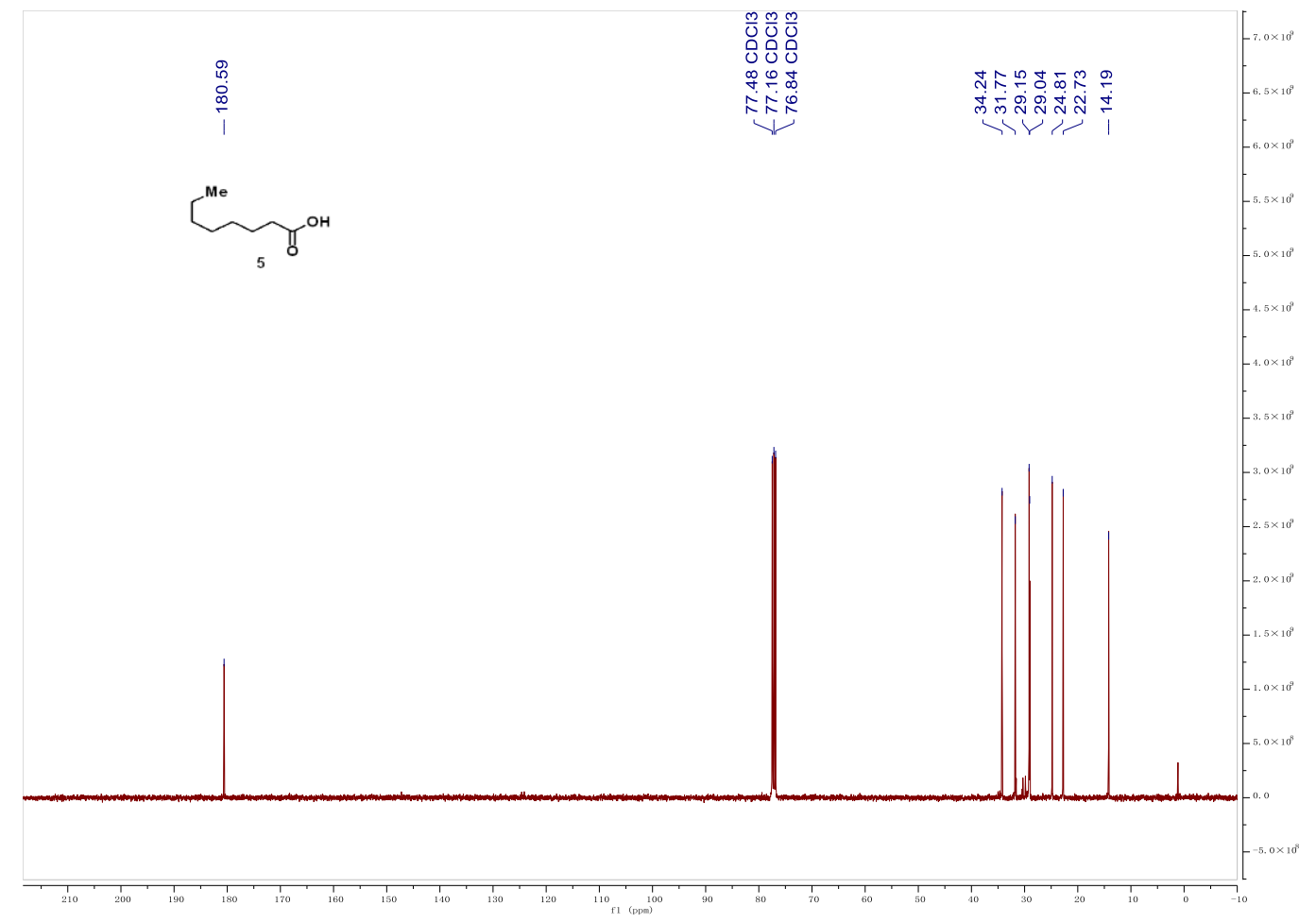




\section{References}

[1] Smith, S. M.; Takacs, J. M. Amide-Directed Catalytic Asymmetric Hydroboration of Trisubstituted Alkenes. J. Am. Chem. Soc. 2010, 132, 1740-1741.

[2] Coyle, E. E.; Doonan, B. J.; Holohan, A. J.; Walsh, K. A.; Lavigne, F.; Krenske, E. H.; O'Brien, C. J. Catalytic Wittig Reactions of Semi- and Nonstabilized Ylides Enabled by Ylide Tuning. Angew. Chem. Int. Ed. 2014, 53, 12907-12911.

[3] Guisán-Ceinos, M.; Soler-Yanes, R.; Collado-Sanz, D.; Phapale, V. B.; Buñuel, E.; Cárdenas, D. J. Ni-Catalyzed Cascade Cyclization-Kumada Alkyl-alkyl Cross-Coupling. Chem. Eur. J. 2013, $19,8405-8410$.

[4] Zhong, Z.; Wang, Z.-Y.; Ni, S.-F.; Dang, L.; Lee, H. K.; Peng, X.-S.; Wong, H. N. C. LigandFree Iron-Catalyzed Carbon $\left(\mathrm{sp}^{2}\right)-$ Carbon $\left(\mathrm{sp}^{2}\right)$ oxidative Homo-Coupling of Alkenyllithiums. Org. Lett. 2019, 21, 700-704.

[5] Chen, Y.; Ma, G.; Gong, H. Copper-Catalyzed Reductive Trifluoromethylation of Alkyl Iodides with Togni’s Reagent. Org. Lett. 2018, 20, 4677-4680.

[6] Karabiyikoglu, S.; Boon, B. A.; Merlic, C. A. Cycloaddition Reactions of Cobalt-Complexed Macrocyclic Alkynes: the Transannular Pauson-Khand Reaction. J. Org. Chem. 2017, 82, 77327744.

[7] Shang, R.; Huang, Z.; Xiao, X.; Lu, X.; Fu, Y.; Liu, L. $\beta$-Aryl Nitrile Construction via PalladiumCatalyzed Decarboxylative Benzylation of $\alpha$-Cyano Aliphatic Carboxylate Salts. Adv. Synth. Catal. 2012, 354, 2465-2472.

[8] Hazra, A.; Lee, M. T.; Chiu, J. F.; Lalic, G. Photoinduced Copper-Catalyzed Coupling of Terminal Alkynes and Alkyl Iodides. Angew. Chem. Int. Ed. 2018, 57, 5492-5496.

[9] Zhao, S.; Mankad, N. P. Cu-Catalyzed Hydroxymethylation of Unactivated Alkyl Iodides with CO to Provide One-Carbon-Extended Alcohols. Angew. Chem. Int. Ed. 2018, 57, 5867-5870.

[10] Barré, B.; Gonnard, L.; Campagne, R.; Reymond, S.; Marin, J.; Ciapetti, P.; Brellier, M.; Guérinot, A.; Cossy, J. Iron- and Cobalt-Catalyzed Arylation of Azetidines, Pyrrolidines, and Piperidines with Grignard Reagents. Org. Lett. 2014, 16, 6160-6163.

[11]Rezazadeh, S.; Devannah, V.; Watson, D. A. Nickel-Catalyzed $C$-Alkylation of Nitroalkanes with Unactivated Alkyl Iodides. J. Am. Chem. Soc. 2017, 139, 8110-8113.

[12]Kitano, Y.; Chiba, K.; Tada, M. A Direct Conversion of Alcohols to Isocyanides. Tetrahedron Lett. 1998, 39, 1911-1912.

[13]Wu, S.; Huang, J.; Gazzarrini, S.; He, S.; Chen, L.; Li, J.; Xing, L.; Li, C.; Chen, L.; Neochoritis, C. G.; Liao, G. P.; Zhou, H.; Dömling, A.; Moroni, A.; Wang, W. Isocyanides as Influenza A Virus Subtype H5N1 Wild-Type M2 Channel Inhibitors. ChemMedChem. 2015, 10, 1837-1845.

[14] Adachi, M.; Miyasaka, T.; Hashimoto, H.; Nishikawa, T. One-Step Transformation of Trichloroacetamide into Isonitrile. Org. Lett. 2017, 19, 380-383.

[15]Mihara, K.; Okada, I.; Chiba, K.; Kitano, Y. Facile Synthesis of N-Substituted Amides from Alkenes and Amides by a Brønsted Acid Mediated Electrophilic Addition Reaction. Synthesis 2014, 46, 1455-1462. 
[16] Kobayashi, G.; Saito, T.; Kitano, Y. A Novel Method for Preparing Isocyanides from NSubstituted Formamides with Chlorophosphate Compounds. Synthesis 2011, 20, 3225-3234.

[17] Jia, S.; Su, S.; Li. C.; Jia, X.; Li, J. Multicomponent Cascade Cycloaddition Involving Tropone, Allenoate, and Isocyanide: A Rapid Access to a 7,6,5-Fused Tricyclic Skeleton. Org. Lett. 2014, $16,5604-5607$.

[18] Kim.; Beebe, J. M.; Jun, Y.; Zhu, X.-Y.; Frisbie, C. D. Correlation between HOMO Alignment and Contact Resistance in Molecular Junctions: Aromatic Thiols versus Aromatic Isocyanides. $J$. Am. Chem. Soc. 2006, 128, 4970-4971.

[19] Evans, D. D.; Evans, D. E.; Palmer, P. J. Estrone Derivatives and Methods for Their Production. GB984029, 1965.

[20] Le, H. V.; Fan, L.; Ganem, B. A Practical and Inexpensive 'Convertible' Isonitrile for Use in Multicomponent Reactions. Tetrahedron Lett. 2011, 52, 2209-2211.

[21] Li, J.; Lear, M. J.; Kawamoto, Y.; Umemiya, S.; Wong, A. R.; Kwon, E.; Sato, I.; Hayashi, Y. Oxidative Amidation of Nitroalkanes with Amine Nucleophiles using Molecular Oxygen and Iodine. Angew. Chem. Int. Ed. 2015, 54, 12986-12990.

[22] Serrano, E.; Martin, R. Nickel-Catalyzed Reductive Amidation of Unactivated Alkyl Bromides. Angew. Chem. Int. Ed. 2016, 55, 1521-3773.

[23] Lücking, U.; Tucci, F. C.; Rudkevich, D.M.; Rebek, J. Self-Folding Cavitands of Nanoscale Dimensions. J. Am. Chem. Soc. 2000, 122, 8880-8889.

[24] Ritter, J. J.; Minieri, P. P. A New Reaction of Nitriles. I. Amides from Alkenes and Mononitriles. J. Am. Chem. Soc. 1948, 70, 4045-4048.

[25] Honkanen, E.; Pippuri, A.; Kairisalo, P.; Nore, P.; Karppanen, H.; Paakkari, I. Synthesis and Antihypertensive Activity of Some New Quinazoline Derivatives. J. Med. Chem. 1983, 26, 14331438.

[26] Zhou, F.; Ding, K.; Cai, Q. Palladium-Catalyzed Amidation of N-Tosylhydrazones with Isocyanides. Chem.-Eur. J. 2011, 17, 12268-12271.

[27] De Kimpe, N.; Verhé, R.; De Buyck, L.; Chys, J.; Schamp, N. Functional Group Transformation. Conversion of Aldehydes into Amides via a-Cyanoenamines. Org. Prep. Proced. Int. 1978, 10, $149-156$.

[28] Owston, N. A.; Parker, A. J.; Williams, J. M. J. Highly Efficient Ruthenium-Catalyzed Oxime to Amide Rearrangement. Org. Lett. 2007, 9, 3599-3601.

[29] Možina, S.; Iskra, J. Aerobic Oxidation of Secondary Alcohols with Nitric Acid and Iron(III) Chloride as Catalysts in Fluorinated Alcohol. J. Org. Chem. 2019, 84, 14579-14586. 